\title{
Integrative Therapy based on Yoga, Ayurveda and Modern Western Medicine for Treatment of High-risk Cases of COVID-19: A Telemedicine-Based Case Series
}

\author{
Sumitra A. Bentur ${ }^{\mathrm{a}}$, Alka Mishra ${ }^{\mathrm{b}}$, Yogesh Kumar $^{\mathrm{c}}$, Sonika Thakral ${ }^{\mathrm{d}, \mathrm{e}, *}$, \\ Sanjiv $^{\mathrm{f}}$, Rahul Garg ${ }^{\mathrm{e}, \mathrm{g}, \mathrm{h}}$ \\ ${ }^{a}$ Ayurveda physician and yoga expert, Noida, India \\ ${ }^{b}$ Department of Ayurveda and Holistic Health, Dev Sanskriti Vishwavidyalaya, Haridwar, \\ India \\ ${ }^{c}$ Advait Clinic, Delhi, India \\ ${ }^{d}$ Shaheed Sukhdev College of Business Studies, University of Delhi, Delhi, India \\ ${ }^{e}$ National Resource Center for Value Education in Engineering, Indian Institute of \\ Technology, Delhi, India \\ ${ }^{f}$ Department of Chemical Engineering, Indian Institute of Technology, Delhi, India \\ ${ }^{g}$ Amar Nath and Shashi Khosla School of Information Technology, Indian Institute of \\ Technology, Delhi, India \\ ${ }^{h}$ Department of Computer Science and Engineering, Indian Institute of Technology, Delhi, \\ India
}

\begin{abstract}
Background: We report a telemedicine-based case-series of thirty high risk COVID-19 positive patients with co-morbidities such as Diabetes Mellitus (DM), Hypertension (HTN), Hypothyroidism, Ulcerative Colitis and Chronic Kidney Disease (CKD) or aged 60 and above (or possibly both), treated successfully using an Integrative Therapy plan based on Ayurveda, Yoga and Modern Western Medicine. The symptoms experienced by the patients included fever, body-ache, sore throat/throat pain, cough/running nose, headache, nasal/chest congestion, stomach problems (such as diarrhea, constipation), nausea, loss of taste, loss of smell, foul smell or taste, loss of appetite, weakness, gastric upset, respiratory trouble (such as breathlessness or high respiration rate) and anxiety. Two patients (both aged above 70) were asymptomatic and remained asymptomatic
\end{abstract}

\footnotetext{
${ }^{*}$ Corresponding author

Email addresses: infobentursuma@yahoo.com (Sumitra A. Bentur), alka.mishra@dsvv.ac.in (Alka Mishra), advaitayurveda@yahoo.co.in (Yogesh Kumar), sthakral@nrcvee.iitd.ac.in (Sonika Thakral), a27070@chemical.iitd.ac.in (Sanjiv), rahulgarg@cse.iitd.ac.in (Rahul Garg)
}

Preprint submitted to International Journal of Infectious Diseases 
during the entire course of the treatment.

Design: The reported cases have been categorized into YAS (patients taking Yoga and Ayurveda based treatment, with possibly western Supplements), YASP (patients taking Yoga and Ayurveda based treatment, with possibly western Supplements and Paracetamol), YAM (patients taking Yoga and Ayurveda based treatment, and Modern western medicine (MWM) as an adjunct), MYA (patients who first tried the Modern western medicines (in most of the cases mandated by the government) and later switched to Yoga and Ayurveda treatment. The last group has been further subdivided into subgroups - patients who developed new symptoms after the commencement of Yoga and Ayurveda based treatment (MYA-S) and those who did not (MYS-NS). The YAS group had 4 patients, YASP had 6 patients, YAM had 6 and MYA had 14 patients (MYA-S having 7 and MYA-NS having 7).

Case-Series Presentation: Ayurveda is a highly personalized system of medicine that considers the Prakruti (the Ayurvedic constitution) as well as Vikruti (the homeostatic imbalances) in treating the patients. Based on the patient's symptoms and co-morbidities, a personalized treatment plan including Ayurvedic medicines, Yoga protocol, dietary recommendations and lifestyle modifications was prescribed by a registered Ayurveda doctor and a Yoga consultant. More than half of the symptomatic patients started experiencing improvement within 5 days (90\% within 9 days) from the start of the treatment. More than $60 \%$ of the symptomatic patients reported at least $90 \%$ recovery within 10 days (90\% within 17 days) from the start of the treatment. Six patients, whose $\mathrm{SpO} 2$ level was observed to be at or below $95 \%$, benefited from the practice of Makarasana and Shithilasana. None of the patients progressed to severe stage of illness. Many patients experienced improvement with respect to their comorbidities besides COVID-related symptoms. Some of them, after recovering from COVID, even sought consultation for long-term management of their comorbidities through Ayurveda and Yoga. Some patients got so convinced about the possibility of restoration of health through Yoga, that they adopted this practice into their lifestyle. 
Conclusions: The Integrative Therapy was found to be very effective in mitigating the symptoms of all the high-risk cases of COVID-19 patients with comorbidities who remained compliant to the treatment. Considering that approximately $32.8 \%$ of the patients with the comorbidities such as DM, HTN, COPD, cardiovascular disease, cerebrovascular disease, chronic kidney disease, Hepatitis B, malignancy, immunodeficiency progress to severe stage of illness [1], and assuming under the null hypothesis that a similar percentage of patients are expected to progress to a severe stage for the treatment population, the corresponding p-value (incorporating 23 symptomatic patients with one or more of the above comorbidities) turns out to be $1.07 \times 10^{-4}$. Integrative Therapy based on classical texts of Ayurveda and Yoga from the East and emergency treatment of modern western medicine may offer a promising and scalable treatment option for COVID-19 patients. More studies including a suitably designed randomized controlled trial is needed to assess its efficacy.

Keywords: SARS-Cov-2, COVID-19, Ayurveda, Yoga, Integrative Therapy

\section{Introduction}

With the rapid rate at which COVID-19 engulfed the planet, this pandemic has become one of the most deadly health disasters witnessed in the known history of mankind. Across 219 countries and territories hit by The deadly

5 coronavirus, more than 113.29 confirmed cases of COVID-19 and more than 2.5 million deaths owing to the disease have been reported as on February 25, 2021 [2]. The case fatality rate (CFR) among COVID-19 patients (as reported on February 25, 2021) worldwide is approximately $2.2 \%$; for India it is $1.4 \%$, for USA $1.7 \%$ and for China $5.1 \%$ [3]. Besides claiming numerous lives, the 10 pandemic crippled healthcare infrastructures and economies across the globe. The severity of illness caused by COVID-19 has been observed to be associated with one's medical condition [4, 5, 6, 7]. COVID-19 patients with co-morbidities such as Diabetes Mellitus (DM), Hypertention (HTN), Chronic Obstructive Pulmonary Disease (COPD) or old age have been observed to exhibit poorer clinical 
outcomes [1, 4, 5, 8, thereby making the management of the illness even more challenging. While the mortality rate for hospitalized COVID-19 patients in China has been reported to be $3.1 \%$ [1], for those amongst these patients having one or more comorbidities, the mortality rate has been reported to be $8.8 \%$ [1]. In the same cohort, the percentage of patients for whom the illness progresses to a severe stage has been reported to be $16 \%$, whereas for patients with co-morbidities, the corresponding percentage is substantially higher at $32.8 \%$. Several research studies aimed at assessing the efficacy of various therapeutic agents for COVID-19 are in progress. For instance, in a double-blind, randomized, placebo-controlled trial conducted on hospitalized adult COVID-19 patients having lower respiratory tract infection, Remdesivir was found superior to placebo in speeding up recovery [9]. Several experimental monoclonal antibody treatments targeting the SARS-CoV-2 S protein are also in the human testing phase, and at least 150 other antibodies are in discovery research [10]. However, no known cure for COVID-19 is yet available in the Modern Western

so Medicine (MWM). Intensive research to find vaccine for the novel coronavirus disease is also in progress. As of February 2021, 66 vaccines are in different phases of trial across the world [11.

Ayurveda and Yoga are two ancient complementary systems of India based on identical theoretical foundations. While Ayurveda focuses on health and well-

35 ness, Yoga assumes basic physical and mental health and proceeds to unfold subtler and deeper aspects of life [12, 13. The system of Yoga adopts a holistic approach to well-being. According to the classical Yogic text 'Yoga Vashishta' [14] (Book 6, Chapter 81), health is freedom from physical diseases as well as from erroneous affections of the mind. The holistic principle of mind-body-soul, which is the foundation of Yogic philosophy, defines a theoretical framework for the human body with five different and progressively subtler layers of abstractions. These layers are termed as Pancha kosha (or five sheaths), viz., Annamaya (physical body), Pranamaya (energy field), Manomaya (mental dimension), Vijnanamaya (related to intuitive knowledge) and Anandamaya (level ${ }_{45}$ of bliss) (Taittiriya Upanishad [15, 16]). The goal of most yogic practices is to 
achieve holistic well-being by ensuring proper distribution and circulation of the vital life force within all realms of existence.

Ayurveda, one of the most ancient holistic healing systems based on profound therapeutic principles, is well-supported with a rich pool of evidence of empirical use [17, 18]. According to Ayurveda, every individual possesses a unique combination of Doshas (Vata, Pitta, Kapha), Agni (metabolism / digestion), Dhatu (body tissues/elements that provide them nourishment) and Mala (excreta) [17, which defines one's prakriti or constitution (Ashtanga Hridaya, Sharira Sthana, Chapter 3, Verse 83) [19]. A stable equilibrium of these constituent elements is the key to good health. Factors such as environment, experiences, emotions, food habits, season, of physical toxins, irritants, micro-organism or impurities, unhealthy habits cause these elements to change, possibly disturbing their equilibrium and causing imbalance (vikruti), which may further manifest as lack of energy, excess mucous, inflammation, and a wide variety of dysfunction and diseases (Ashtanga Hridaya, Sharira Sthana, Chapter 3, Verse 83) [19]. Ayurveda works on the principle of restoring this balance according to one's prakruti and vikruti, making it intrinsically personalized.

Interestingly, classical Ayurveda texts such as Charaka Samhita (Vimana Sthana, ${ }_{65}$ Chapter 3) [18] and Sushruta Samhita (Sutra Sthana, Chapter 6, Verse 19, 20) [17] describe COVID-like pandemics, referring to them as Janapadoddhvansa, literally translating into 'destruction of communities'. They also offer specific guidelines about treatment and management of diseases that spread during Janapadoddhvansa. Based on this, many proposals to use Ayurveda for prophylaxis 20, 21, 22, 23, 24, 25, 26, 27, 28, 29, 30, 31, 32, 33. as well as treatment of COVID-19 34, 22, 35, 36, 37, 23, 24, 25, 27, 38, 28, 39, 40, 41, 42, 43, have been published. See [44] (Related Works) for a detailed review on use of Yoga, Ayurveda and other systems of traditional medicine for treatment of COVID-19. More recently, there are reports of the use of Ayurveda for prophylaxis as well as 75 treatment of COVID-19. In an open-label randomized controlled trial performed on COVID patients with mild to moderate symptoms in a tertiary care facility 
[45], the Ayurvedic medicines Dasamoolkaduthrayam Kashaya and Guluchyadi Kwatham (in tablet form) were administered as adjunct to the Standard of Care treatment to the treatment group; the Ayurvedic regime was observed to have fastened recovery in terms of reduction of symptoms (particularly breathlessness) as well as duration of stay in hospital. In another randomized controlled trail [40] that used the Ayurvedic medicine Guduchi Ghan Vati in addition to the standard treatment for asymptomatic COVID patients, all the patients in the intervention group continued to remain asymptomatic while $11.7 \%$ of those in the control group developed mild symptoms; moreover, significant differences between the two groups were observed in terms of duration of hospital stay as well as virologic clearance on days 7 and 14. The first COVID case treated entirely with Ayurveda has also been reported [46. In another case report [47, very promising result of Ayurvedic treatment was seen on a COVID patient with severe hypoxia. The patient whose $\mathrm{SpO} 2$ remained at $80 \%$ even with oxygen supplementation not only voluntarily opted for Ayurveda but also refused to take any allopathic medicines except Vitamin C. The patient showed improvement within a day of the start of Ayurvedic treatment with no breathlessness and stable $\mathrm{SpO} 2$ between $95 \%$ and $98 \%$; oxygen supplementation was removed within 2 days. A recent study 44 reports the use of Yoga and Ayurveda in the treatment of a high-risk case of COVID-19.

The case series presented here is the first major study on thirty high-risk COVID-19 positive patients with co-morbidities such as Diabetes Mellitus (DM), Hypertension (HTN), Hypothyroidism, Ulcerative Colitis and Chronic Kidney Disease (CKD) or aged 60 and above (or possibly both), treated successfully using an Integrative Therapy plan based on Ayurveda, Yoga and Modern Western Medicine. The cases form a rich diversity that includes patients taking Yoga and Ayurveda based treatment, with possibly western Supplements (YAS), patients taking Yoga and Ayurveda based treatment, with possibly western Supplements and Paracetamol (YASP), patients taking Yoga and Ayurveda based treatment, and Modern western medicine (MWM) as an adjunct (YAM), patients who first tried the Modern western medicines (in most of the cases mandated by the gov- 
ernment) and later switched to Yoga and Ayurveda treatment (MYA). The MYA group had patients who developed new symptoms after the commencement of Yoga and Ayurveda based treatment (MYA-S) and those who did not (MYSNS). The YAS group had 4 patients, YASP had 6 patients, YAM had 6 and MYA had 14 patients (MYA-S having 7 and MYA-NS having 7). The Ayurveda and Yoga treatment protocol was customized to the constitution (prakturi) and needs (vikruti) of the patients, in conformance with the principles of Ayurveda. All the patients reported benefit from the Integrative therapy and none of them progressed to the severe stage of the illness.

The case series presented here adds to the already growing evidence in support of the use of Yoga and Ayurveda for treatment and management of COVID-19. The authors are not aware of any published work reporting the use of Ayurveda or Yoga in the successful treatment of high-risk cases of COVID-19 besides a single-case study [44]. The present case-series offers a sufficiently strong evidence supporting that incorporating the elements of Yoga and Ayurveda in the treatment plan of high-risk cases of COVID-19 with mild to moderate symptoms can be very promising. A suitably designed randomized control trial (RCT), while incorporating the personalized treatment principles of Ayurveda and Yoga, would be strongly advised to take the full advantage of these ancient sciences while searching for a viable and effective cure for the COVID-19 pandemic.

\section{Methods}

\subsection{Patients and Enrollment}

Patients were recruited into the study based on the following inclusion criteria:

- Willingness to participate in the study and provide the informed consent

- Willingness to share all the details (such as government-issued photo id.) required for telemedicine practice 
- Access to video conference capability (phone/computer with high speed Internet) for consultations, Yoga and monitoring

- Confirmed cases of COVID-19 with age between 18 and 60 years and presenting history of one or more of the following comorbidities: diabetes, hypertension, COPD, CAD, malignancy.

- Confirmed cases of COVID-19 with age 60-75 years without any comorbidities

Enrolments to the case series began on July 1, 2020 and went on till November 8, 2020. A total of 47 patients filled in the registration form to join the study (online or offline); out of these 13 patients were dropped due to non-compliance, one patient was excluded from the study since she had no symptoms at the time of enrolment and turned negative within 2 days of the first consultation, three patients were dropped because they did not satisfy the inclusion criteria. We hereby present the case reports of 30 patients. Table 1 presents the demographic details and other relevant information about the patients whose case reports have been presented in this paper.

\subsection{Treatment Settings}

The presented case series involved treatment through telemedicine. Only confirmed positive cases of COVID-19 were recruited into the study; for a patient to get enrolled into the study, he/she was required to fill in a registration form (online or hard-copy) and sign an informed consent. A detailed medical history and existing condition of the patient was subsequently recorded, based on which a personalised treatment plan (based on Ayurveda and Yoga) was prescribed by the concerned Ayurveda doctor and the yoga therapist in the team. In case a patient with COVID-like symptoms approached one of the Ayurveda doctors in the team, Ayurveda-based symptomatic treatment was initiated, but the patient was enrolled in the study only after being detected positive with COVID; the treatment plan was also appropriately revised thereafter. The treatment prescribed to the patients comprised Ayurvedic medicines, 10-15 guided Yoga 
tients enrolled in the study could have had taken or be simultaneously taking modern western medicine for COVID in consultation with other doctor(s). Also, the patients continued with their ongoing treatments for any comorbidity(ies) they had.

170

The patients recruited in the study have been classified into the following categories based on their intake of modern western medicine for COVID-19: (i) YAS: only Yoga-and-Ayurveda-based treatment with possibly allopathic supplements (ii) YASP: Yoga-and-Ayurveda-based treatment with allopathic supplements and paracetamol (iii) YAM: Yoga-and-Ayurveda-based treatment with modern western medicine as adjunct (iv) MYA: Yoga-and-Ayurveda-based treatment pursued after modern western medicine; this category has been further divided into three subcategories, viz. MYA-S (new symptoms presented after enrolment into the study), MYA-NS (no new symptoms presented after enrolment into the study) and MYA-A (asymptomatic throughout).

For the purpose of this study, the day when a patient receives the first consultation from a doctor in our team would be considered as the 'date of first consultation' for the patient. Consultation with the Ayurveda doctor and Yoga therapist was done through telephone and/or via video call (as per requirement); the yoga sessions were held through video calls (skype/google meet/whatsapp). Regular follow-up was done with the patients and the patients could also approach the Yoga therapist as well as their consulting Ayurveda doctor in the team. At the end of the guided sessions, the patients were given a recorded video of the yoga protocol for them to continue practising on their own.

As per the protocol of the study, any patient becoming severe during the course of the treatment (characterized by dyspnea, hypoxia, severe lung infection or other severe symptoms as assessed by the Ayurveda doctors and yoga thera195 
(though no such situation arose during the execution of the study). It may be noted that the ICMR guidelines for testing and treatment of COVID-19 kept evolving during the study. For instance, initially, ICMR had mandated a second RTPCR test to confirm absence of infection before terminating the treatment and isolation period of a patient even after recovery from symptoms [4]. Later, the norms changed and COVID patients who had recovered from symptoms and had been quarantined for a stipulated period of time no longer needed a second test [49]. In line with the government-issued guidelines, the treatment concluded with the patients' symptoms being (almost) resolved and their isolation period ending. Most patients chose not to go for a second test, but some did. The authors have reported the information as it was. No funding from any source was received for carrying out the presented case series. No fee was charged for consultation or guided yoga sessions; however, the patients had to buy their own medicines and pay for the suggested/required tests.

\section{Case Presentations}

This section provides an overall picture of the study. The number of patients in each of the categories described above is as follows: YAS had 4 patients, YASP and YAM had 6 each, and MYA had 14 patients with 7 in MYA-S, 7 in MYANS. Two of the cases were asymptomatic, one of which was from YAS and the other from MYA; both the patients remained asymptomatic throughout. An overview (including the comorbidities and medical history of the patients, and the progression of illness with respect to COVID-19) of the cases enrolled in the study follows.

\subsection{Comorbidities, Present-Illnesses and Medical History}

Table 2 summarizes the comorbidities and medical history of the patients enrolled. 


\subsection{History of Present Illness/COVID}

Table 3 summarizes the history of present illness (COVID) - the date of first consultation, category of the patient, the onset and progression of symptoms,

\subsection{Treatment Plan}

The pool from which Ayurvedic medicines were prescribed to the patients is given in Section 3.3.1 and the generic yoga protocol based on which personalised protocols were designed is given in Section 3.3.2 Besides Ayurvedic medicines the patients. An important practice recommended to many patients was nasya (putting 2 drops of oil in each nostril) with anu teil/mustard oil/almond oil/ghee made with cow-milk. Nasya is a purification procedure in the Panchakarma Therapy of Ayurveda wherein herbal medicines in different forms (oil, powder the administered medicine to be absorbed by the Shringataka marma (a vital spot at the union of the nerves in the head region that supplies to the nose, ears, eyes, and tongue) thereby affecting the supra-clavicular region [50. Some other common suggestions given to the patients are listed below: usha paan 240 (drinking 2-3 glasses of lukewarm water in the morning and drinking lukewarm water throughout the day, taking steam (with Ajwain/turmeric/rock salt added to water or with plain water), doing gargles, adopting spiritual practices such as meditation, chanting and Yoga nidra for which links were provided (51], 52], [53, [54]). Also, dietary recommendations were given that included turmeric milk at night, consuming light/easy to digest food, increase in the intake of fruits and green vegetables, coconut water; refraining from cold, sour, fried, spicy, heavy to digest food such as curd, cold drinks, ice-creams, chilled water, any refrigerated food items, black gram pulse (or any food item prepared with black gram), jackfruit etc. 


\subsubsection{Pool of Ayurvedic medicines prescribed to patients}

The prescriptions given to patients comprised suitable Ayurvedic medicines from the following pool (besides other components of the therapy): Giloy Ghanvati, Sanshamani vati, Ashwagandha vati, Lavangadi vati, Chitrakadi vati, Arogyavardhini vati, Oritus syrup, Pathyadi Kwath - pravahi, Laghumalini Vasant, Sudarshan Ghanvati, Mahasudarshan Ghanvati, Kutaj Ghanvati, Laxmivilas ras, Basant Kusumakar ras, Immunity capsules, Bacnil capsules, Fifatrol tablet, Zoemit tablet, Bowel care, Softovac, Septilin tablet, Amynity plus syrup, Himcocid syrup, Diarex syrup, Koflet-SF syrup, Kutajarishta, Jirakadyarishta, Vasavaleh, Jufex Forte, Chyawanprash, Ayush Kwath, Nagaradi Kwath, Kantakari Avaleha, Talishadi churna, Amalaki churna, Aloe-vera+amla juice, Swarna vasant malti ras, Diabecon, Sitopaladi, Madhumeh kusumakar ras, Vijaysar churna, Astha 15 syrup, Astha 15 capsules, Amlapittantak yog, Alsarex, Himcospaz, Bresol, Sarpagandha vati, Coldab tablets, Kufrakshak syrup, Immunocare tablets, Cardiol-H tablets, Unexozim Forte tablets, Orplat tablets, Oro-T.

\subsubsection{Components of the yoga protocols designed for patients}

The common components in the yoga protocols were: Sukshma Vyayama (subtle joint movements for upper and lower body parts); breathing exercises; asanas (a subset from the following set were included based on each patient's condition and capability: Tadasana, Triyaktadasana, Trikonasana, Hastapadasana, Ardhachakrasana, Virabhadrasana, Parshva Sukhasana, Sukhasana twist, Utthana Mandukasana, Ardha Ushtrasana, Vakrasana, Meru Vakrasana, Bhujangasana, Shalabhasana, Ardha Halasana - with one leg folded, Anantasana, Pawan Muktasana, Matsyasana), supported Supta-vajrasana; Shavasana (quick relaxation/deep relaxation); pranayama (Sectional breathing and full Yogic breathing, Anulom-vilom, Chandra/Surya Anulom-vilom, Bhramari, Udgeet, ); Dhyana (mindful breathing).

The Yoga component of the Integrative Therapy was tailored for each patient to achieve maximum benefits considering the co-morbidities and COVID-19 symp- 
to the patients have been provided in supplementary material, justifying their use.

\section{Results}

The offered treatment delivered very promising outcomes. Out of the 23 symptomatic and comorbid patients in the case series, each with one or more of following conditions - DM, HTN and CAD, no one's symptoms turned severe; no patient required hospitalization or needed oxygen supplementation. Given that approximately $32.8 \%$ of the patients with the above comorbidities are expected 
to progress to severe stage of illness [1], the corresponding p-value (incorporating treatment (and illness). The day of the first consultation with an Ayurveda doctor has been considered as Day0. The intensity of symptoms has been marked on a 4-point scale with 0 meaning absence of symptom and 1 being the maximum intensity; 0.33 and 0.66 are intermediate levels. Average cumusymptoms observed for each patient was computed for each day after the commencement of the treatment; this measure was averaged over 28 symptomatic patients and plotted against the respective day. The symptoms experienced by the patients included fever, body-ache, sore throat/throat pain, cough/running 
Average Cumulative Intensity of Symptoms per Patient

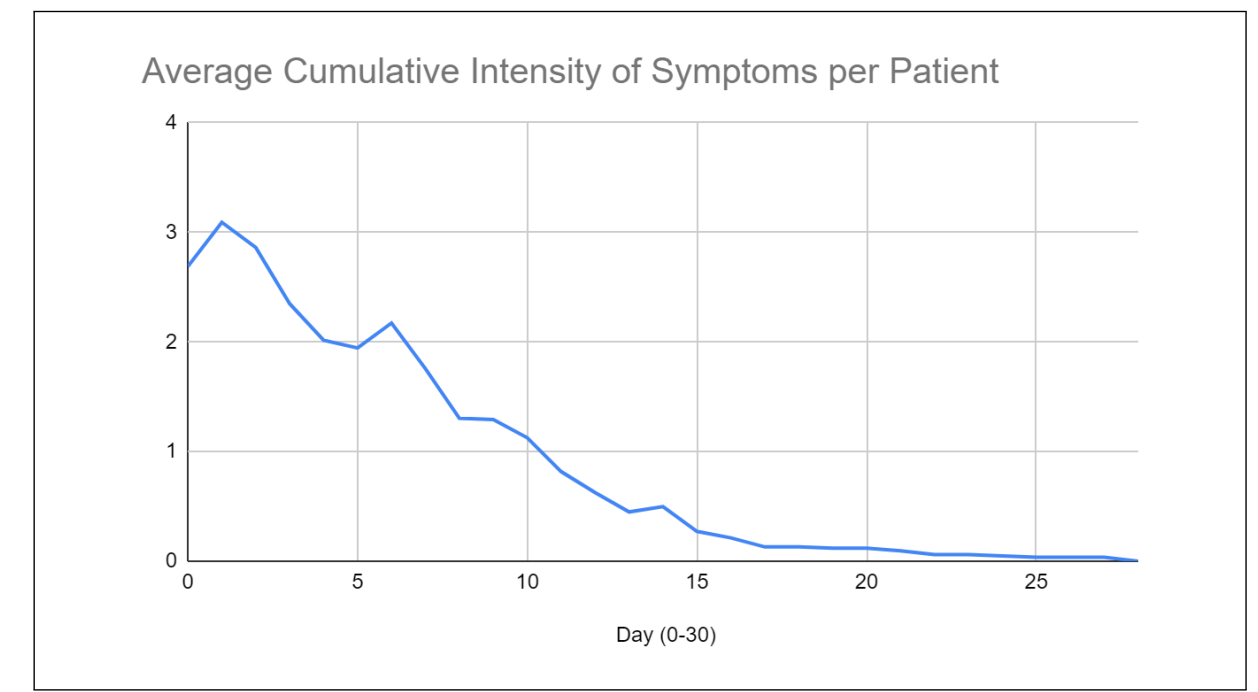

Figure 1: Average cumulative intensity of symptoms per patient with progression of treatment.

nose, headache, nasal/chest congestion, stomach problems (such as diarrhea, constipation), nausea, loss of taste, loss of smell, foul smell or taste, loss of appetite, weakness, gastric upset, respiratory trouble (such as breathlessness or high respiration rate) and anxiety. An overall idea of the efficacy of the treatment may be derived from the following statistics. More than $53 \%$ of the symptomatic patients started experiencing improvement within 5 days and almost $90 \%$ of them within 9 days from the start of the treatment. More than $60 \%$ of the symptomatic patients reported at least $90 \%$ recovery (approximately) within 10 days and almost 90\% reported the same within 17 days from the start of the treatment (with some of these patients reporting complete recovery while others reporting almost complete recovery).

Table 4 summarizes the result of each case undertaken; this comprises the date by which most symptoms were resolved, the symptoms that got resolved by this date and those that persisted. 


\section{Discussion and Conclusions}

This study reports a telemedicine-based case series of 30 high-risk COVID-19 patients successfully treated using integrative therapy based on Ayurveda, Yoga and modern western medicine. To the best of the authors' knowledge, this is

the first report on the use of Ayurveda and Yoga in the treatment of high-risk cases of COVID-19. Remarkable results were observed with $100 \%$ recovery rate; the patients not only recovered from COVID-related symptoms, but also experienced improvement with respect to their comorbidities, illness-induced anxiety and oxygen saturation levels. Many patients who experienced improvement with respect to their comorbidities with Ayurveda-and-Yoga-based intervention consulted the Ayurveda doctors in the team for long-term management. Many patients were so convinced about the role Yoga played in accelerating their recovery and bestowing an overall sense of well-being post-illness that they got committed to adopting Yoga in their lifestyles. Another important observation was the commendable improvement in the oxygen saturation levels of patients who had a tendency of SpO2 level dropping below 95. Yet another dimension where Yoga gave an edge to the overall treatment was controlling the anxiety of patients. It is well-known that COVID patients, go through a lot of distress due to the uncertainty associated with the disease; this is worsened by the fear of spreading it to other family members, staying in isolation and the associated social stigma owing to the highly contagious nature of the disease. To add to all this, the patients treated in the presented case series were all high-risk patients with one or more comorbidities and/or old age. As can be expected, most of the patients exhibited anxiety initially, which could weaken their already compromised immune system and worsen their symptoms thereby creating a vicious circle. However, authors believe that regular practice of yoga (comprising asanas, pranayama, deep relaxation, dhyana) came to the rescue. Some patients reported to be reaping immense benefits from the practice of Yoga nidra. The authors attribute the success of the treated high-risk cases to the holistic approach adopted by Ayurveda and Yoga; not only were the symptoms 
managed, but the immune system was strengthened and the psychologically affected patients were imparted relief through a sense of overall well-being.

The case fatality rate (CFR) for COVID-19 in India has been reported to be 390 around $1.4 \%[3]$ which is significantly lower than that of many other countries [3]. The recently released third National Sero-Survey of India [57] suggests that the COVID-19 may have much wider spread in India than previously thought. Interestingly, this data may also suggest a significantly lower COVID-19 infection fatality rate for the Indian population. The use of Ayurveda and Yoga by the Indian population may be an important factor which needs to be carefully examined while explaining the lower COVID-19 IFR in India. Ayurveda, Traditional Indian Medicine (TIM) based on Ayurveda, and Yoga are very widely practised in India.

The Ministry of AYUSH, Government of India issued advisories for the use of Ayurveda and Yoga to boost immunity, manage and prevent COVID-19 [58, 59]. The study [60] has already demonstrated the role of Yoga in lowering the stress during the COVID-19 lockdown. A suitably designed cross sectional study may be able to uncover the impact of Yoga and Ayurveda in the infection fatality rate of COVID-19 in India.

${ }_{405}$ As on July 14, 2020, 25\% of the total 312 Indian clinical trials registered with CTRI involved Ayurveda, Yoga and Naturopathy [61. While the results of some of these trials (such as [45, 40]) indicate positive results for Ayurvedic formulations, most of these trials restrict to studying the impact of one formulation on the entire patient population without following the true Ayurvedic approach of personalized medicine. This severely limits the potential benefits that Ayurvedic and Yogic sciences have to offer for COVID-19. The World Health Organization and Ministry of AYUSH propose the black-box approach for clinical trials in Traditional Medicines [62, 63]. Unfortunately, executing a clinical trial involving the traditional Indian systems of Yoga and Ayurveda 415 while adopting the black-box approach has its own set of administrative challenges, which is beyond the scope of this paper. The case series presented in 
this paper demonstrates how personalized treatment plan comprising Yoga and Ayurveda and modern western medicine can be very effective in treating highrisk cases of COVID-19. However, in order to establish the effectiveness of the black-box approach, suitably designed clinical trials are required.

The crisis, known as 'COVID', that humanity is facing today needs an immediate, scalable, affordable and easy to administer solution. This situation has made us realise that times to come may bring more health hazards in different forms. Thus, it is not just the novel coronavirus that we need to fight. The solution lies in preparing ourselves by strengthening our immune system and and working towards physical, emotional and psychological well-being. Given the need of the hour, the traditional Indian systems of Yoga and Ayurveda deem fit. When the entire world is suffering, India may have an invaluable treasure to offer. The promising results of the presented case series pose the need for a well-designed randomized controlled trial to conclusively establish the efficacy of Yoga and Ayurveda for the current pandemic.

\section{Acknowledgements}

We would like to express sincere gratitude to Dr. Saurabh Mishra, Dev Sanskriti Vishwavidyalaya, for his continued guidance and valuable suggestions. We immensely benefited from his all-rounded expertise. We also acknowledge the help and support extended by Prof. Bharat Bajirao Bhoyar, Chaudhary Brahma Prakash Ayurved Charak Sansthan. Last, but not the least, we thank Prof. Bhanu Duggal, AIIMS Rishikesh for her valuable inputs.

\section{Declarations}

Ethics Approval and Consent to Participate

No explicit ethics approval was required for this case-series as it was conducted in compliance with the notification from the Ministry of AYUSH for undertaking research on COVID 19 through Ayurveda, Unani, Siddha and Homeopathy systems 64] and the Telemedicine Practice Guidelines for Ayurveda, Siddha and 
to the commencement of the treatment, an informed consent for participation was duly taken from each subject.

\section{Consent for Publication}

A consent for publishing the anonymized details was duly taken from all the

\section{References}

[1] W.-j. Guan, W.-h. Liang, Y. Zhao, H.-r. Liang, Z.-s. Chen, Y.-m. Li, X.-q. Liu, R.-c. Chen, C.-l. Tang, T. Wang, et al., Comorbidity and its impact on 1590 patients with covid-19 in china: A nationwide analysis, European Respiratory Journal 55 (5).

[2] Worldometer, Countries where COVID-19 has spread, retrieved February 25, 2021 from https://www.worldometers.info/coronavirus/countrieswhere-coronavirus-has-spread/.

[3] Worldometer, COVID-19 Coronavirus Pandemic, retrieved February 25, 2021 from https://www.worldometers.info/coronavirus/\#countries.

[4] B. Wang, R. Li, Z. Lu, Y. Huang, Does comorbidity increase the risk of patients with COVID-19: evidence from meta-analysis, Aging (Albany NY) 12 (7) (2020) 6049.

[5] A. K. Singh, R. Gupta, A. Misra, Comorbidities in COVID-19: Outcomes in hypertensive cohort and controversies with renin angiotensin system blockers, Diabetes \& metabolic syndrome: Clinical Research \& Reviews.

[6] O. A. Espinosa, A. d. S. Zanetti, E. F. Antunes, F. G. Longhi, T. A. d. Matos, P. F. Battaglini, Prevalence of comorbidities in patients and mortality cases affected by SARS-CoV2: a systematic review and metaanalysis, Revista do Instituto de Medicina Tropical de São Paulo 62. 
[7] R. Franki, Comorbidities Increase COVID-19 Deaths by Factor of 12, medscape. Retrieved August 12, 2020 from https://www.medscape.com/viewarticle/932486 (2020).

[8] L. Zhu, Z.-G. She, X. Cheng, J.-J. Qin, X.-J. Zhang, J. Cai, F. Lei, H. Wang, J. Xie, W. Wang, et al., Association of Blood Glucose Control and Outcomes in Patients with COVID-19 and Pre-existing Type 2 Diabetes, Cell Metabolism.

[9] J. Beigel, K. Tomashek, L. Dodd, et al., Remdesivir for the treatment of covid-19 - final report.[published online october 8, 2020], N Engl J Med. doi 10 .

[10] L. DeFrancesco, Covid-19 antibodies on trial, Nature biotechnology 38 (11) (2020) 1242-1252.

[11] Wikipedia, Coronavirus disease 2019, retrieved February 25, 2021 from https://en.wikipedia.org/wiki/Coronavirus_disease_2019.

[12] U. Nations, Resolution adopted by the General Assembly on 11 December 2014. 69/131. International Day of Yoga, retrieved August 20, 2020 from https://en.wikisource.org/wiki/Page:United_Nations_Resolution_No._6913_(International_Day_of_Yoga).djvu/1 (January 9, 2015).

[13] D. G.L.Verma, Patanjali Yoga Sutra, Chaukhamba Surbharati Prakashan, 490 2010.

[14] R. P. Arya, The Yoga Vasistha of Valmiki, Chaukhamba Sanskrit Pratishthan, 2005.

[15] S. Sharvananda, Taittiriya-Upanishad, The Ramakrishna Math, Mylapore Madras, 1921.

495 [16] S. N. Saraswati, Prana and Pranayama, Yoga Publications Trust, 1 December 2009. 
[17] K. R. S. Murthy, Susruta Samhita, Varanasi, Uttar Pradesh, India: Chaukhambha Orientalia, 2008.

[18] P. V. Sharma, Caraka Samhita, Varanasi, Uttar Pradesh, India: Chaukhambha Orientalia, 2008.

[19] R. Vidyanath, Illustrated Astanga Hrdaya, Chaukhambha Sanskrit Pratishthan, 2018.

[20] G. Tillu, S. Chaturvedi, A. Chopra, B. Patwardhan, Public Health Approach of Ayurveda and Yoga for COVID-19 Prophylaxis, The Journal of Alternative and Complementary Medicine 26 (5) (2020) 360-364.

[21] R. P. Rajkumar, Ayurveda and COVID-19: Where psychoneuroimmunology and the meaning response meet, Brain, Behavior, and Immunity.

[22] B. Patwardhan, P. Chavan-Gautam, M. Gautam, G. Tillu, A. Chopra, S. Gairola, S. Jadhav, Ayurveda rasayana in prophylaxis of COVID-19, Current Science 118 (2020) 1158-1160.

[23] S. S. K. Goothy, S. Goothy, A. Choudhary, G. Potey, H. Chakraborty, A. H. Kumar, V. Mahadik, Ayurveda's Holistic Lifestyle Approach for the Management of Coronavirus disease (COVID-19): Possible Role of Tulsi, International Journal of Research in Pharmaceutical Sciences 11 (SPL1) (2020) 16-18.

[24] S. Niraj, S. Varsha, A review on scope of immuno-modulatory drugs in Ayurveda for prevention and treatment of Covid-19, Plant Science Today 7 (3) (2020) 417-423.

[25] A. Nandan, S. Tiwari, V. Sharma, Exploring alternative medicine options for the prevention or treatment of coronavirus disease 2019 (COVID-19)-A systematic scoping review, medRxiv.

[26] D. S. Rajput, Evolution, Ayurveda, immunity, and preventive aspects for emerging infectious diseases such as COVID-19, International Journal of Research in Pharmaceutical Sciences 11 (SPL1) (2020) 86-93. 
[27] B. Mittal, T. Chand, et al., Global Care through Ayurveda in Pandemic of COVID-19, International Journal of Health Sciences and Research 10 (6) (2020) 165-172.

[28] S. Kar, B. Gurubasavaraja, S. Vikaram, K. Sriharsha, R. Deshpande, Ayurvedic Preventive and Curative Protocol for COVID 19-A Proposal, Journal of Ayurveda and Integrated Medical Sciences (ISSN 2456-3110) 5 (2) (2020) 92-108.

[29] A. D. Majumdar, V. M. Soni, N. Munjal, Ayurveda and Yoga: An Immunity Booster at the Time of COVID-19 Pandemic, International Journal of Research in Engineering, Science and Management 3 (6).

[30] G. A. Kulkarni, CONCEPTUAL STUDY OF AAHARA VIDHI VISHESHA AYATANA IN PANDEMIC DISEASE COVID-19, Journal of Ayurveda and Integrated Medical Sciences (ISSN 2456-3110) 5 (3) (2020) $75-79$.

[31] H. Soni, S. Sharma, J. K. Malik, Synergistic Prophylaxis on COVID-19

[35] A. K. Panda, A. K. Dixit, S. Rout, B. Mishra, U. V. Purad, S. Kar, Ayurveda Practitioners Consensus to Develop Strategies for Prevention 
and Treatment of Corona Virus Disease (COVID-19), Journal of Ayurveda and Integrated Medical Sciences (ISSN 2456-3110) 5 (1) (2020) 98-106.

[36] B. Vellingiri, K. Jayaramayya, M. Iyer, A. Narayanasamy, V. Govindasamy, B. Giridharan, S. Ganesan, A. Venugopal, D. Venkatesan, H. Ganesan, et al., COVID-19: A promising cure for the global panic, Science of The Total Environment (2020) 138277.

[37] G. Tillu, S. Salvi, B. Patwardhan, AYUSH for COVID-19 management, Journal of Ayurveda and Integrative Medicine 11 (2) (2020) 95.

[38] A. J. Gandhi, J. D. Rupareliya, V. Shukla, S. B. Donga, R. Acharya, An Ayurvedic Perspective along with in Silico Study of the Drugs for the Management of Sars-Cov-2, Journal of Ayurveda and Integrative Medicine.

[39] R. Jajal, V. Dive, Probable Protocol for Clinical Management of COVID19 With Ayurvedic Treatment Modalities Adjuvant to Ongoing Conventional Treatment - A Review, International Journal of Research in Ayurveda and Medical Sciences 3 (2020) 132-137.

[40] A. Kumar, G. Prasad, S. Srivastav, V. K. Gautam, N. Sharma, A retrospective study on efficacy and safety of guduchi ghan vati for covid-19 asymptomatic patients, medRxiv.

[41] B. P. Stella, S. S. Kokatnur, S. Desai, Understanding of COVID-19 in Ayurveda and Its Managing View, Journal of Ayurveda and Integrated Medical Sciences (ISSN 2456-3110) 5 (2) (2020) 161-165.

[42] B. Ozah, Triphala: A useful therapeutic supplement during COVID-19 pandemic, Journal of Drug Delivery and Therapeutics 10 (4) (2020) 219220.

[43] S. Roy, P. Bhattacharyya, Possible role of traditional medicinal plant Neem (Azadirachta indica) for the management of COVID-19 infection, International Journal of Research in Pharmaceutical Sciences 11 (SPL1) (2020) 122-125. 
[44] A. Mishra, S. A. Bentur, S. Thakral, R. Garg, B. Duggal, The use of integrative therapy based on yoga and ayurveda in the treatment of a high-risk case of covid-19/sars-cov-2 with multiple comorbidities: a case report, Journal of Medical Case Reports 15 (1) (2021) 1-12.

[45] P. Wanjarkhedkar, G. Sarade, B. Purandare, D. Kelkar, A prospective clinical study of an ayurveda regimen in covid 19 patients, Journal of Ayurveda and Integrative Medicine.

[46] P. Girija, N. Sivan, Ayurvedic treatment of COVID-19/SARS-CoV-2: A case report, Journal of Ayurveda and Integrative Medicine.

[47] J. A. Joshi, R. Puthiyedath, Outcomes of ayurvedic care in a covid-19 patient with hypoxia-a case report, Journal of Ayurveda and integrative medicine.

[48] Discharge Policy of $\mathrm{nCoV}$ Case, Ministry of Health and Family Welfare. Retrieved February 27, 2021 from https://www.mohfw.gov.in/pdf/Corona\%20Discharge-Policy.pdf (March 2020).

[49] Revised Discharge Policy for COVID-19, Ministry of Health and Family Welfare. Retrieved February 27, 2021 from https://www.mohfw.gov.in/pdf/ReviseddischargePolicyforCOVID19.pdf (May 2020).

[50] A. Mishra, L. Batham, S. Verma, S. Mishra, V. Shrivastava, Management of epileptic seizures through an integrated approach including yagya therapy, Interdisciplinary Journal of Yagya Research 2 (1) (2019) 52-64.

[51] DSVV, Spiritual Practices Recommended during Present Times, department of Ayurveda and Holistic Health. Dev Sanskriti Vishwavidyalaya, Haridwar. Retrieved August 26, 2020 from https://sites.google.com/dsvv.ac.in/dahh-dsvv/notices/spiritualpractices-present-times (2020). 
[52] Yoga Nidra by Swami Niranjanananda Saraswati, retrieved September 26, 2020 from https://youtu.be/iAX_rQPOIAQ (2016).

[53] Yoga Nidra by Swami Niranjanananda Saraswati, retrieved September 26, 2020 from https://youtu.be/RMrVVfIMOTE (2020).

[54] G. by Swami Niranjanananda Saraswati, Yoga Nidra - Intermediate, retrieved September 26, 2020 from https://youtu.be/WN6q9xQHojI (06 Aug 2013).

[55] D. R. N. Dr. H R Nagendra, New Perspectives In Stress Management, Swami Vivekananda Yoga Prakashana, June 1986.

[56] S. S. Saraswati, Yoga Nidra, 6th edition, Yoga Publications Trust, Munger, Bihar, India, 2015.

[57] S. the Wire, COVID-19: What the Third National Sero-Survey Result Does and Doesn't Tell Us, Retrieved February 25, 2021 from https://science.thewire.in/health/third-national-seroprevalence-surveyicmr-covid-19-rural-prevalence-test-positivity/ (February 05, 2021).

[58] Ministry of AYUSH, Ayurveda's immunity boosting measures for self care during COVID 19 crisis, retrieved August 11, 2020 from https://www.mohfw.gov.in/pdf/ImmunityBoostingAYUSHAdvisory.pdf.

[59] V. R. Kotecha, Advisory From Ministry of AYUSH for Meeting the Challenge Arising Out of Spread of Corona Virus (COVID-19) in India, Secretary, Ministry of AYUSH, Government of India. Retrieved August 11, 2020 from https://www.ayush.gov.in/docs/125.pdf (March 6, 2020).

[60] P. S. Sahni, K. Singh, N. Sharma, R. Garg, Yoga an effective strategy for self-management of stress-related problems and wellbeing during COVID19 lockdown: A cross-sectional study, PloS one 16 (2) (2021) e0245214. 
[69] C. B. Jha, Ayurvediya Rasashastra, Varanasi, India: Chaukhambha Surabharati Prakashan, 2000.

[62] World Health Organization, General Guidelines for Methodologies on Research and Evaluation of Traditional Medicine, (page 12), Retrieved February 27, 2021 from http://apps.who.int/iris/bitstream/handle/10665/66783/WHO_EDM_TRM _2000.1.pdf;jsessionid=43FDA9828FE80959ACB55C03CC788EA4? sequence=1.

[63] Central Council for Research in Ayurvedic Sciences Ministry of AYUSH, Government of India, General Guidelines for Clinical Evaluation of Ayurvedic Interventions, (page 15), Retrieved February 27, 2021 from http://www.ccras.nic.in/sites/default/files/viewpdf/Publication/CCRAS_ Guideline\%20of\%20Clinical_Evaluation.pdf.

[64] Government of India, Ministry of AYUSH, Notification from the Ministry of AYUSH for undertaking research on Covid 19 through Ayurveda, Unani, Siddha and Homeopathy systems, retrieved September 12, 2020 from https://www.ayush.gov.in/docs/127.pdf (April 21, 2020).

[65] Central Council of Indian Medicine, Telemedicine practice guidelines for ayurveda, siddha and unani practitioners, retrieved September 12, 2020 from https://www.ayush.gov.in/docs/CCIM_Telemedicine_Guidelines.pdf (April 7, 2020).

[66] P. V. Sharma, Dravyaguna Vijnana, volume II (vegetable drugs) (Hindi), Chaukhambha Bharati Academy, Varanasi, Uttar Pradesh, India, 2001.

[67] Sandu Pharmaceuticals Ltd., Pathyadi kadha, retrieved August 8, 2020 from http://www.sandu.in/pathyadi.html.

[68] Himalaya, Diabecon, retrieved August 8, 2020 from https://himalayawellness.in/products/diabecon. 
[70] Divya, Lavangadi Vati, patanjali. Retrieved October 8, 2020 from https://www.patanjaliayurved.net/product/ayurvedicmedicine/vati/lavangadi-vati/91.

[71] Divya, Chitrakadi Vati, patanjali. Retrieved October 8, 2020 from https://www.patanjaliayurved.net/product/ayurvedicmedicine/vati/chitrakadi-vati/33.

[72] Divya, Arogyvardhini Vati, patanjali. Retrieved October 8, 2020 from https://www.patanjaliayurved.net/product/ayurvedicmedicine/vati/arogyavardhini-vati/13.

[73] Oritus Syrup, orn Remedies Pvt Ltd. Retrieved October 8, 2020 from https://www.1mg.com/otc/oritus-syrup-otc323261.

[74] Dhootapapeshwar Laghumalini Vasant Rasa, shree Dhootapapeshwar Ltd. Retrieved October 8, 2020 from https://www.1mg.com/otc/dhootapapeshwar-laghumalini-vasant-rasaotc369207.

[75] Sudarshan Ghanvati, zandu. Retrieved October 8, 2020 from https://www.amazon.in/Zandu-Sudarshan-Ghanvati-Pack2/dp/B01LWRK7OF.

[76] Divya Mahasudarshan Ghan Vati, patanjali. Retrieved October 8, 2020 from https://www.1mg.com/otc/patanjali-divya-mahasudarshanghan-vati-otc387949.

[77] Kutaja Ghan Vati, netmeds. Retrieved October 8, 2020 from 685 https://www.netmeds.com/health-library/post/kutaja-ghana-vatibenefits-uses-method-dosage-and-side-effects.

[78] Laxmivilas Ras, patanjali. Retrieved October 9, 2020 from https://www.patanjaliayurved.net/product/ayurvedic-medicine/parpatiras/laxmivilas-ras/92. 
[79] Basant Kusumakar Ras, baidyanath. Retrieved October 9, 2020 from https://www.1mg.com/otc/baidyanath-basant-kusumakar-ras-withgold-silver-pearl-otc326981.

[80] Fifatrol, aIMIL Pharmaceuticals. Retrieved October 9, 2020 from https://www.aimilpharmaceuticals.com/product/fifatrol/.

[81] Zoemit, zoetic. Retrieved October 9, 2020 from http://zoeticayurvedics.com/product_detail.php?ref=27.

[82] Himalaya Septilin Tablet, himalaya Drug Company. Retrieved October 9, 2020 from https://www.1mg.com/otc/himalaya-septilin-tablet-otc191717.

[83] Amynity Plus Syrup, aIMIL. Retrieved October 9, 2020 from https://www.bgr-34.life/products/amynity-plus-syrup-pack-of2 ? variant $=35542469279911$.

[84] Himalaya Himcocid Sugar Free Suspension Banana, himalaya Drug Company. Retrieved October 9, 2020 from https://www.1mg.com/otc/himalaya-himcocid-sugar-free-suspensionbanana-otc125773.

[85] Himalaya Diarex Syrup, himalaya Drug Company. Retrieved October 9, 2020 from https://www.1mg.com/otc/himalaya-diarex-syrup-otc275646.

[86] Himalaya Koflet-SF, himalaya Drug Company. Retrieved October 9, 2020 from https://www.1mg.com/otc/himalaya-koflet-sf-linctus-otc321546.

[87] Kutajarishta, patanjali. Retrieved October 9, 2020 from https://www.patanjaliayurved.net/product/ayurvedicmedicine/arishta/kutajarishta/85.

[88] , Baidyanath Jirakadyarishta, ayurveda Bay. Retrieved October 9, 2020 from https://www.ayurvedabay.com/baidyanath-jirakadyarishta.html.

[89] Vasavaleh, ayur Healers. Retrieved October 9, 2020 from https://ayurheals.com/product/vasavaleh/. 
[90] Jufex Forte, aimil Pharmaceuticals. Retrieved October 13, 2020 from https://www.aimilpharmaceuticals.com/product/jufex-forte/.

[91] Ayush Kwath, jiva Ayurveda. Retrieved October 13, 2020 https://store.jiva.com/products/ayush-kwath-ayurvedic-immunityenhancer/.

[92] Nagaradi Kashayam, retrieved October 13, 2020 from https://www.ayurmedinfo.com/2015/08/12/nagaradi-kashayam/.

[93] Kantakari Avaleha, retrieved October 13, 2020 from https://www.ayurmedinfo.com/2012/02/09/kantakari-avaleha-usesdosage-side-effects-and-ingredients/.

[94] Talisadi Churna, ayur Times. Retrieved October 13, 2020 from https://www.ayurtimes.com/talisadi-churna-ingredients-benefits-dosageside-effects/.

[95] Amalaki Churna, planet Ayurveda. Retrieved October 13, 2020 from https://www.planetayurveda.com/library/amalaki-churna/.

[96] Jiva Amla + Aloe Vera Juice, jiva Ayurveda. Retrieved October 13, 2020 from https://store.jiva.com/products/jiva-amla-aloe-juice/.

[97] Swarn Vasant Malti Ras, basic Ayurveda. Retrieved October 13, 2020 from https://www.1mg.com/otc/basic-ayurveda-swarn-vasant-malti-raswith-gold-otc450401.

[98] Nagarjun Sitopaladi Tablet, health Mug. Retrieved December 18, 2020 from https://www.healthmug.com/product/nagarjun-sitopaladi-tablet$50 \mathrm{~g} / 1036793550$.

740 [99] Madhumeha Kusumakar Rasa, retrieved December 18, 2020 from https://sdlindia.com/madhumeha-kusumakar-rasa.html.

[100] Vijaysar: Benefits, Uses, Formulation, Ingredients, Dosage And Side Effects, netmeds. Retrieved December 18, 2020 from 
https://www.netmeds.com/health-library/post/vijaysar-benefits-usesformulation-ingredients-dosage-and-side-effects.

[101] Astha-15 Syrup, retrieved December 18, 2020 from https://www.saravanamarundhagam.in/productview/MTAyMw==/d5da2710927c913ad0c5e7c6ed3dede82db28cb7/DAstha-15+Syrup.

[102] Astha-15 Syrup, retrieved December 18, 2020 from https://www.1mg.com/otc/astha-15-syrup-otc377717.

[103] Dalmia Astha 15 Capsules Box Capsule 120 no.s, retrieved December 18, 2020 from https://www.snapdeal.com/product/dalmia-astha-15-capsulesbox/649702041459.

[104] Baidyanath Amlapittantak Yog 100 Tablet, retrieved December 18, 2020 from https://www.medlife.com/baidyanath-amlapittantak-yog-100tablet/otc/BAIDYTB02A.

[105] Himalaya Bresol tablet, retrieved December 19, 2020 from https://www.1mg.com/otc/himalaya-bresol-tablet-otc131450.

[106] Sarpagandha Vati, retrieved December 19, 2020 from https://www.deepayurveda.com/product/sarpagandha-vati/.

[107] Coldab Tablets, retrieved December 21, 2020 from http://www.dhanvantari.co.in/our-products/patent-products/coldabtablets/.

[108] Kufrakshak Syrup, retrieved December 21, 2020 from https://www.1mg.com/otc/kufrakshak-syrup-otc325735.

[109] BACFO Immunocare Tablet, retrieved December 21, 2020 from https://www.1mg.com/otc/bacfo-immunocare-tablet-otc328719. 
[110] Cardio-H Capsules: Uses, Dose, Ingredients, retrieved December 21, 2020

from https://www.ayurmedinfo.com/2015/11/03/cardio-h-capsules-usesdose-ingredients/.

[111] Unexozim Forte Capsules (100 Capsules), retrieved December 21, 2020 from https://www.madanapalas.com/unexozim-forte-capsules-capsulesp-3251.html.

[112] Organic India Immunity Veg Capsule, retrieved January 17, 2021 from https://www.1mg.com/otc/organic-india-immunity-veg-capsuleotc328959.

[113] Orplat Capsule, retrieved December 21, 2020 from https://www.saveonmedicals.com/orplat-capsule-som.

[114] Dhootapapeshwar Sanshamani Vati, retrieved January 15, 2021 from https://www.1mg.com/otc/dhootapapeshwar-sanshamani-vatiotc393192.

[115] Bacnil Capsules - 100 cap Natural antibiotic for G.I. Infections, retireved January 16, 2021 from https://prachinayurvedkutir.com/bacnil-capsules100-cap.html.

[116] Softovac Bowel Regulator Powder, lupin Ltd. Retrieved January 17, 2021 from https://www.1mg.com/otc/softovac-bowel-regulator-powderotc158465.

[117] Organic India Chyawanprash, organic India. Retrieved January 17, 2021 from https://www.1mg.com/otc/organic-india-chyawanprash-otc339446.

[118] Charak Alsarex Tablet, charak Pharma Pvt. Ltd. Retrieved January 17, 2021 from https://www.1mg.com/otc/charak-alsarex-tablet-otc314641.

[119] Himalaya Himcospaz Capsule, himalaya Drug Company. Retrieved January 17, 2021 from https://www.1mg.com/otc/himalaya-himcospazcapsule-otc125774. 
[120] Himalaya Oro-T Oral Rinse, himalaya Drug Company. Retrieved February 27, 2021 from https://himalayawellness.in/products/oro-t-oral-rinse.

\section{Tables}

\begin{tabular}{|c|c|c|c|c|c|c|}
\hline $\begin{array}{l}\text { Patient } \\
\text { Id. }\end{array}$ & Age & Gender & $\begin{array}{l}\text { Height } \\
(\mathrm{cm}) \\
\text { approx }\end{array}$ & $\begin{array}{l}\text { Weight } \\
(\mathrm{Kg}) \\
\text { approx }\end{array}$ & City & $\begin{array}{l}\text { Date of enrolment } \\
\text { (year: 2020) }\end{array}$ \\
\hline P01 & 55 & $\mathrm{M}$ & 160.02 & 70 & Delhi & July 01 \\
\hline $\mathrm{P} 02$ & 40 & $\mathrm{M}$ & 165.02 & 85 & Delhi & July 09 \\
\hline P03 & 62 & $\mathrm{M}$ & 173.00 & 68 & Delhi & July 30 \\
\hline P04 & 75 & $\mathrm{~F}$ & 160.02 & 66 & Delhi & Aug 02 \\
\hline P05 & 65 & $\mathrm{M}$ & 170.18 & 73 & Delhi & Aug 10 \\
\hline P06 & 65 & $\mathrm{~F}$ & 157.48 & 68 & Delhi & Aug 10 \\
\hline $\mathrm{P} 07$ & 38 & $\mathrm{M}$ & 170.00 & 68 & Delhi & Aug 15 \\
\hline P08 & 43 & $\mathrm{M}$ & 160.00 & 95 & Delhi & Aug 25 \\
\hline P09 & 49 & $\mathrm{M}$ & 172.50 & 96 & Delhi & Aug 26 \\
\hline $\mathrm{P} 10$ & 43 & $\mathrm{~F}$ & 165.00 & 77 & Delhi & Aug 27 \\
\hline $\mathrm{P} 11$ & 38 & $\mathrm{M}$ & & & Delhi & Aug 31 \\
\hline $\mathrm{P} 12$ & 45 & $\mathrm{~F}$ & 154.00 & 68 & Delhi & Sep 04 \\
\hline $\mathrm{P} 13$ & 60 & $\mathrm{M}$ & 167.64 & 48 & Delhi & Sep 04 \\
\hline $\mathrm{P} 14$ & 75 & M & 150.00 & 60 & Delhi & Sep 07 \\
\hline $\mathrm{P} 15$ & 66 & $\mathrm{~F}$ & 160.02 & & Delhi & Sep 11 \\
\hline $\mathrm{P} 16$ & 52 & $\mathrm{M}$ & 172.72 & 80 & Delhi & Sep 24 \\
\hline $\mathrm{P} 17$ & 39 & M & 165.10 & 67 & Delhi & Sep 26 \\
\hline $\mathrm{P} 18$ & 49 & M & 177.80 & 86 & Delhi & Sep 30 \\
\hline P19 & 71 & $\mathrm{M}$ & & & Delhi & Sep 30 \\
\hline P20 & 40 & $\mathrm{~F}$ & 157.48 & 71 & Delhi & Oct 12 \\
\hline P21 & 38 & $\mathrm{M}$ & & & Delhi & Oct 16 \\
\hline $\mathrm{P} 22$ & 71 & $\mathrm{M}$ & 162.56 & 56 & Delhi & Oct 22 \\
\hline $\mathrm{P} 23$ & 62 & $\mathrm{~F}$ & 160.02 & 65 & Delhi & Oct 24 \\
\hline $\mathrm{P} 24$ & 35 & $\mathrm{~F}$ & 157.48 & 78 & Delhi & Oct 24 \\
\hline $\mathrm{P} 25$ & 65 & $\mathrm{~F}$ & 162.56 & 70 & Delhi & Oct 26 \\
\hline P26 & 65 & $\mathrm{M}$ & 170.18 & 60 & Delhi & Nov 03 \\
\hline $\mathrm{P} 27$ & 60 & $\mathrm{~F}$ & 157.48 & 70 & Delhi & Nov 03 \\
\hline P28 & 52 & $\mathrm{M}$ & 162.56 & 75 & Delhi & Nov 07 \\
\hline P29 & 44 & $\mathrm{~F}$ & 154.94 & 75 & Delhi & Nov 08 \\
\hline P30 & 55 & $\mathrm{M}$ & 162.56 & 70 & Delhi & Nov 08 \\
\hline
\end{tabular}

Table 1: Patient Details 


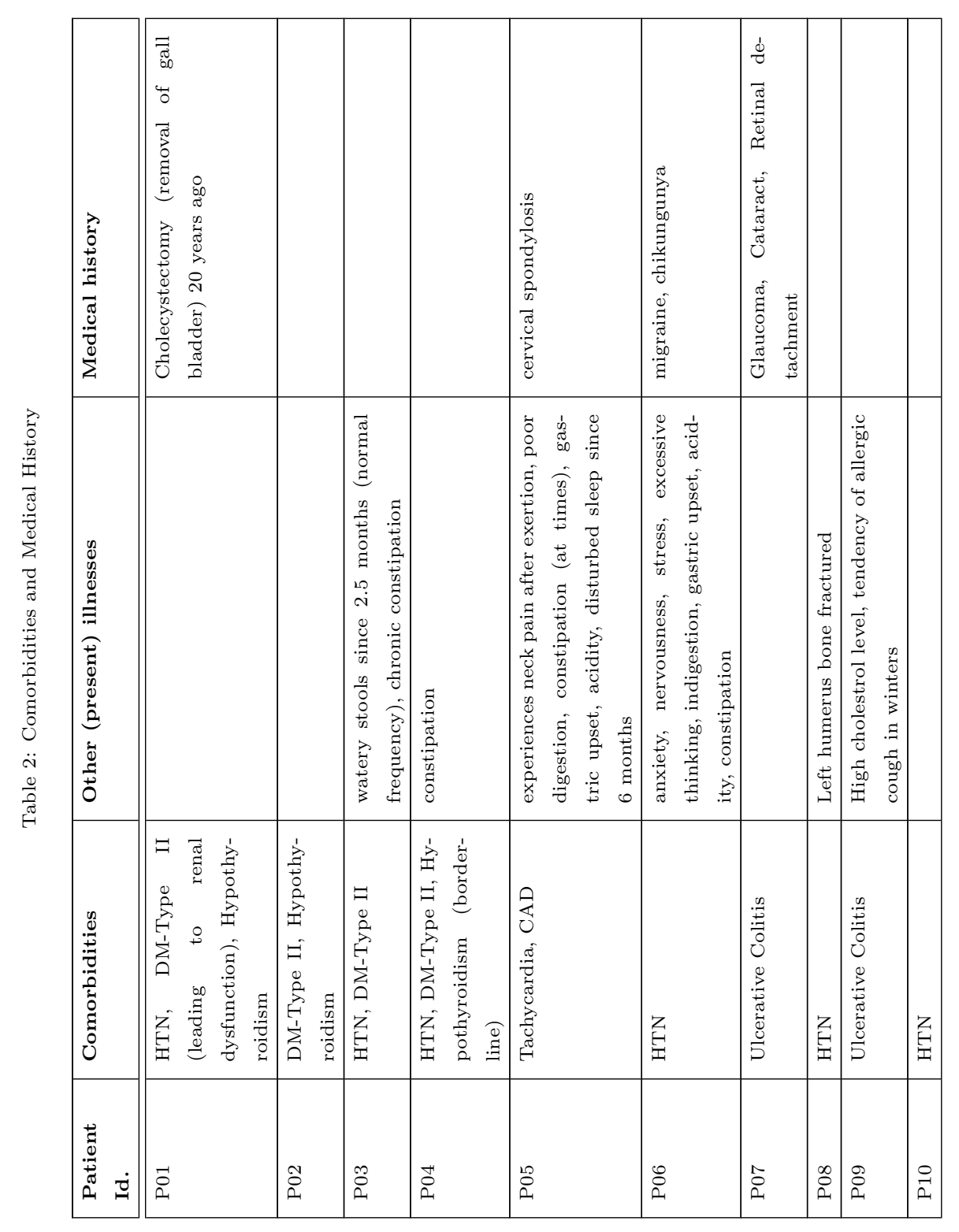




\begin{tabular}{|c|c|c|c|c|c|c|c|c|c|c|c|}
\hline & & 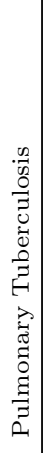 & & 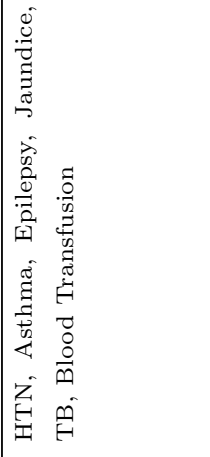 & & 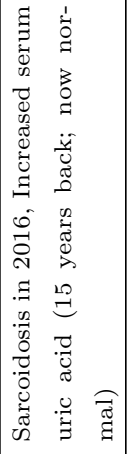 & 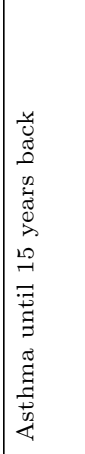 & & & & 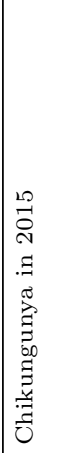 \\
\hline 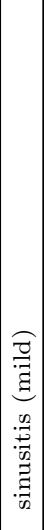 & & 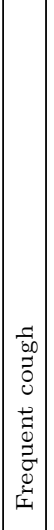 & 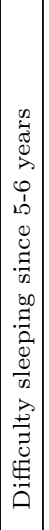 & 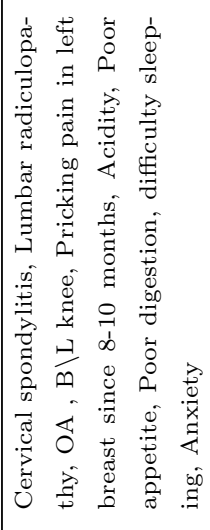 & 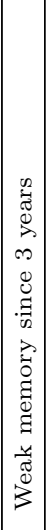 & 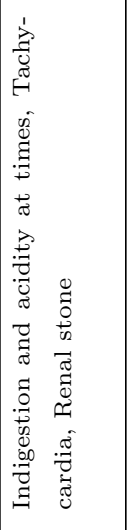 & 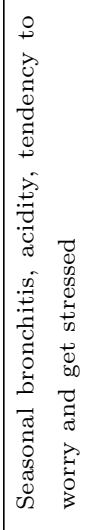 & 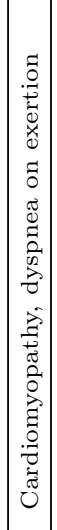 & 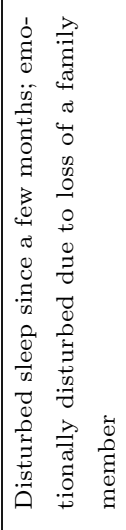 & 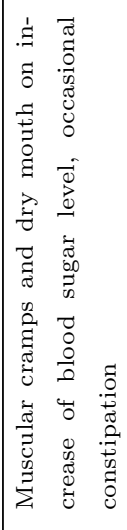 & 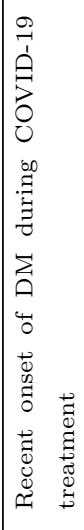 \\
\hline 总 & 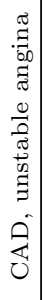 & $\mid \begin{array}{l}0 \\
\tilde{0} \\
\check{z}\end{array}$ & 莺 & 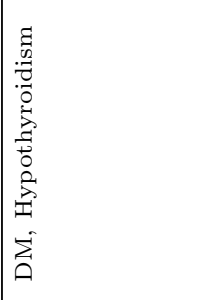 & $\vec{\Delta}$ & 䏍 & 能 & 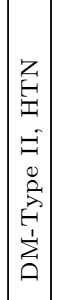 & 窇 & 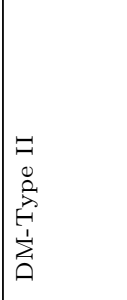 & \begin{tabular}{|l|l} 
\\
$\ddot{\tilde{z}}$ \\
$z$
\end{tabular} \\
\hline $\overrightarrow{\vec{a}_{1}}$ & $\vec{a}$ & $\mid \frac{\infty}{2}$ & $\vec{a}$ & $\vec{a}$ & $\bar{\sigma}$ & $\vec{a}$ & $\overrightarrow{\vec{a}}$ & $\frac{\Omega}{\Omega}$ & 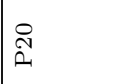 & $\overrightarrow{\tilde{L}}$ & $\tilde{\Sigma}$ \\
\hline
\end{tabular}




\begin{tabular}{|c|c|c|c|c|c|c|c|}
\hline 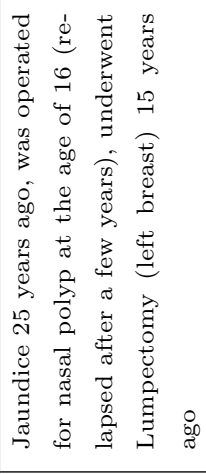 & 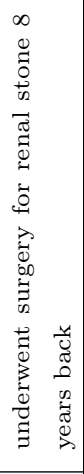 & 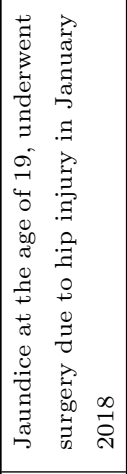 & & & & 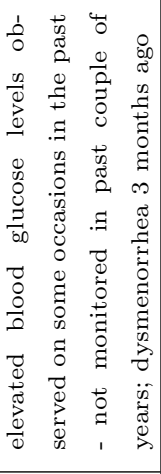 & \\
\hline 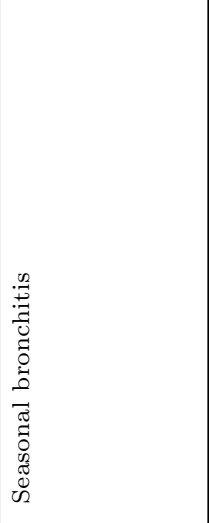 & & 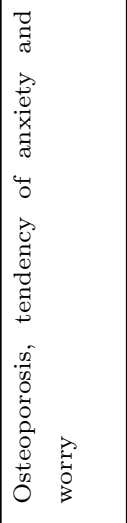 & 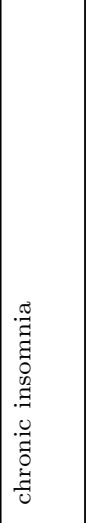 & 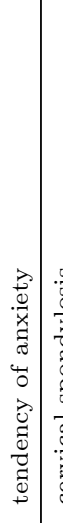 & 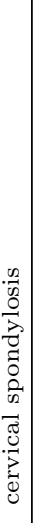 & & \\
\hline 号 & 忘 & 忘 & 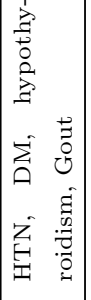 & $\mid$ & & 忘 & 学 \\
\hline ڤ̊ & $\vec{\Sigma}$ & ڤ̃ & 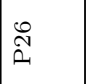 & $\begin{array}{c}\hat{N} \\
\stackrel{\omega}{2}\end{array}$ & ָे & ڤે & $\ddot{\mathscr{m}}$ \\
\hline
\end{tabular}




\begin{tabular}{|c|c|c|c|c|c|}
\hline 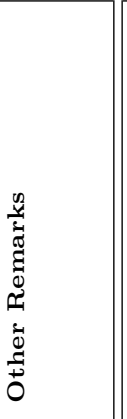 & 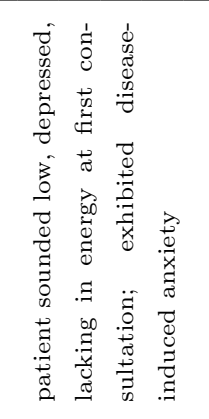 & 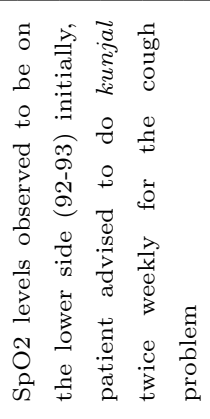 & 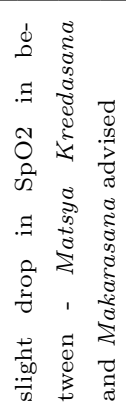 & 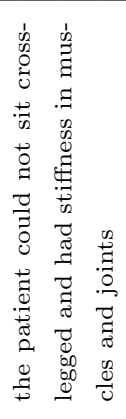 & \\
\hline 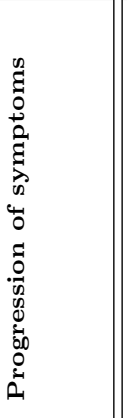 & 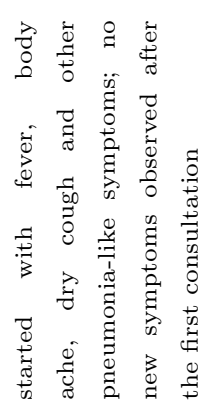 & 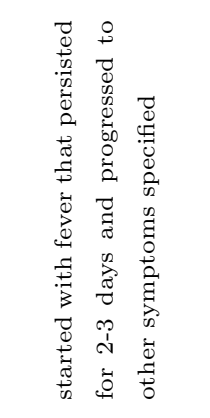 & 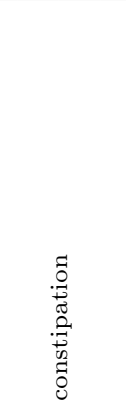 & 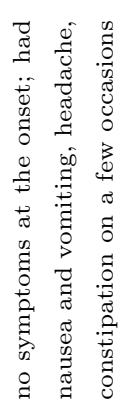 & 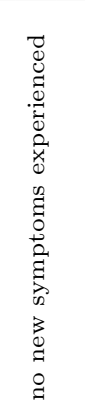 \\
\hline 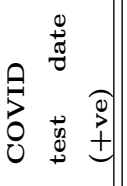 & $\begin{array}{l}\vec{N} \\
\Xi \\
\Xi \\
\Xi\end{array}$ & $\vec{H}$ & 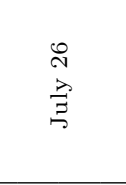 & $\begin{array}{l}\vec{\infty} \\
\vec{\Xi}\end{array}$ & $\begin{array}{l}\infty \\
\infty \\
ٍ\end{array}$ \\
\hline 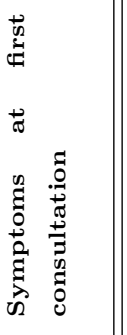 & 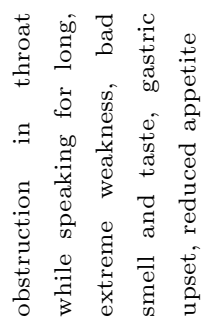 & 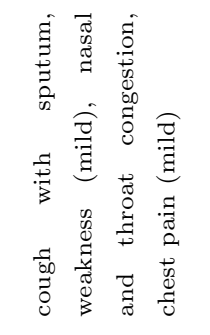 & 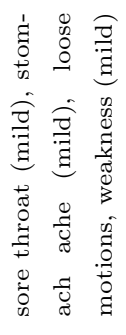 & 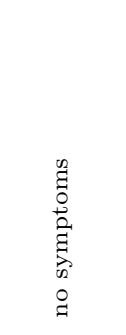 & 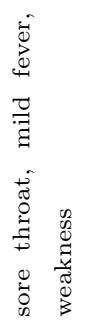 \\
\hline 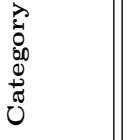 & $\sum_{\substack{n \\
\sum}}^{n_{2}}$ & $\sum_{\substack{n \\
\vdots}}^{\frac{n}{4}}$ & 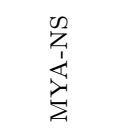 & $\sum_{\lambda}^{\infty}$ & 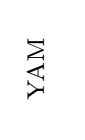 \\
\hline 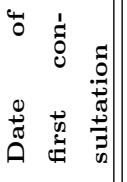 & $\overrightarrow{3}$ & $\begin{array}{l}0 \\
\vec{\Xi} \\
\vec{g}\end{array}$ & 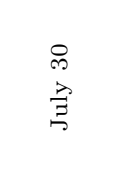 & $\begin{array}{l}\overrightarrow{6} \\
\stackrel{6}{2}\end{array}$ & 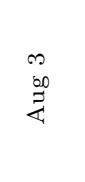 \\
\hline 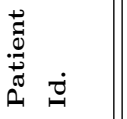 & $\overrightarrow{0}$ & $\begin{array}{c}\tilde{o} \\
\tilde{\alpha}\end{array}$ & $\begin{array}{l}\infty \\
\dot{\alpha}\end{array}$ & 苍 & 总 \\
\hline
\end{tabular}




\begin{tabular}{|c|c|c|c|c|}
\hline & 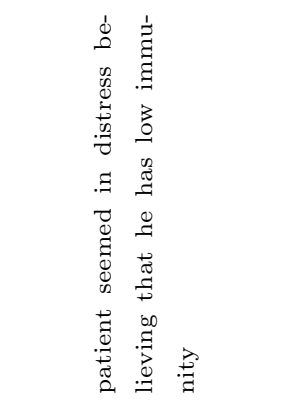 & 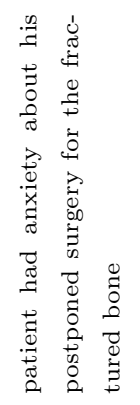 & 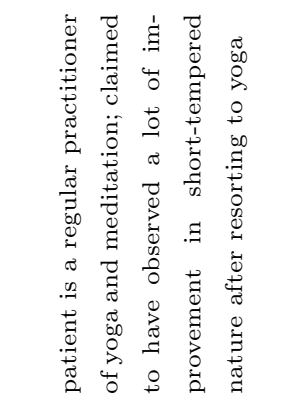 & 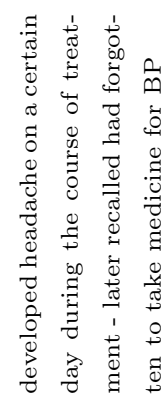 \\
\hline 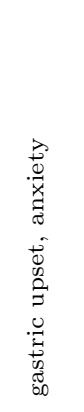 & 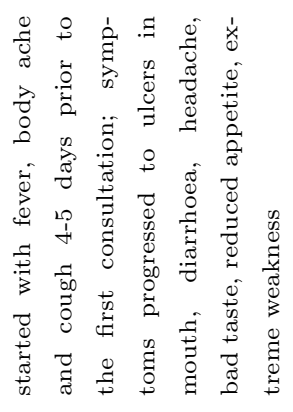 & 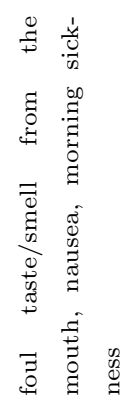 & 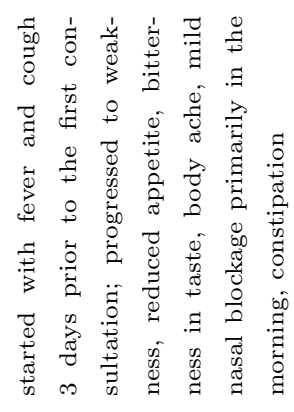 & 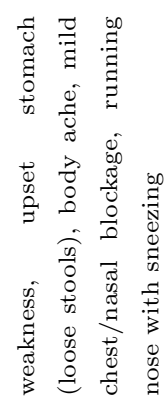 \\
\hline 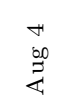 & 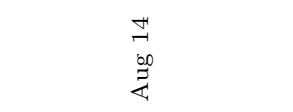 & $\begin{array}{l}\vec{N} \\
60 \\
\sum\end{array}$ & 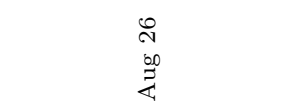 & 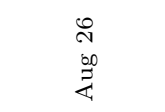 \\
\hline 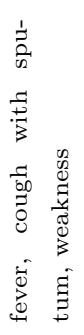 & 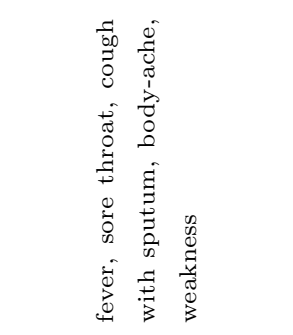 & 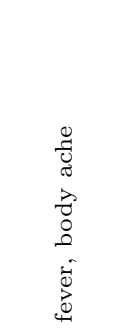 & 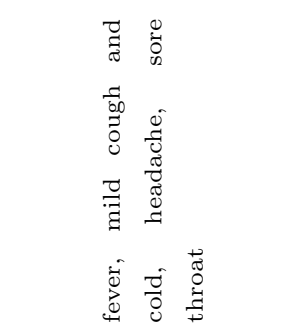 & 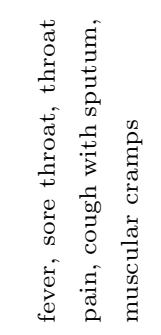 \\
\hline$\sum_{\lambda}$ & $\sum_{\substack{i \\
\sum}}^{\infty}$ & $\sum_{\lambda}^{\infty}$ & $\sum_{\substack{n \\
\sum}}^{\infty}$ & 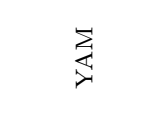 \\
\hline $\begin{array}{l}+4 \\
\stackrel{\infty}{\sharp} \\
\Xi\end{array}$ & $\begin{array}{l}20 \\
\stackrel{0}{0} \\
\stackrel{6}{4}\end{array}$ & 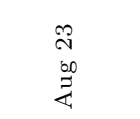 & $\begin{array}{l}\infty \\
\stackrel{1}{0} \\
\infty \\
\Xi\end{array}$ & $\begin{array}{l}\stackrel{\wedge}{A} \\
\text { so } \\
\Xi\end{array}$ \\
\hline \& & $\begin{array}{l}\hat{\circ} \\
a\end{array}$ & $\stackrel{\infty}{a}$ & \&े & $\stackrel{ }{\vec{Q}}$ \\
\hline
\end{tabular}




\begin{tabular}{|c|c|c|c|c|c|}
\hline 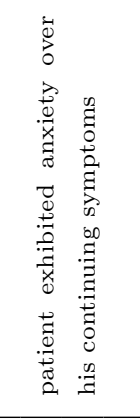 & 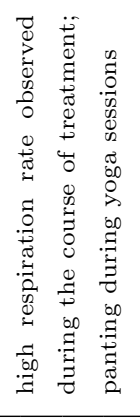 & & 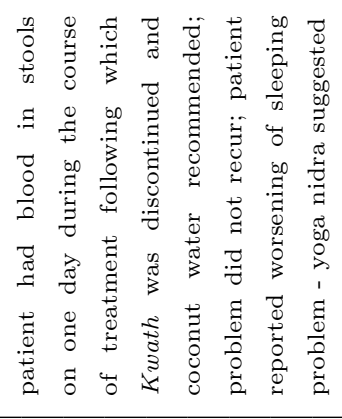 & 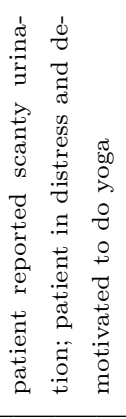 & \\
\hline 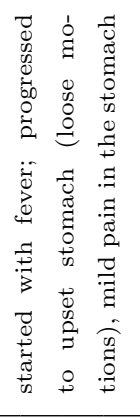 & 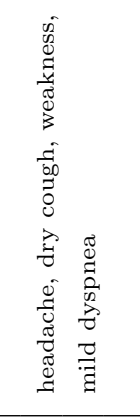 & 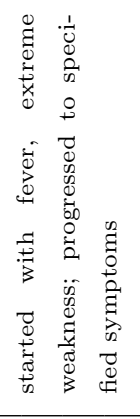 & 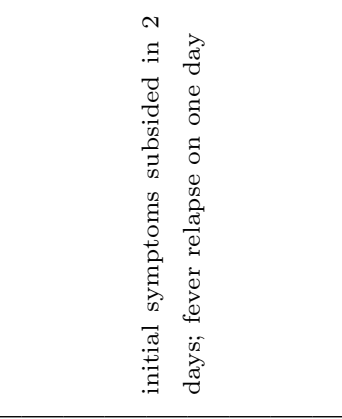 & 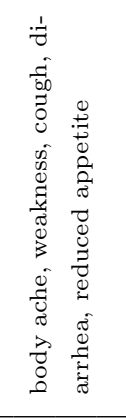 & 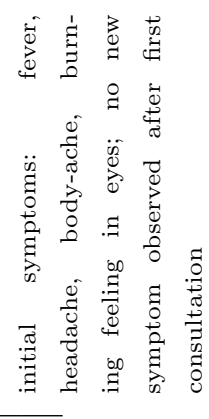 \\
\hline $\begin{array}{l}0 \\
\stackrel{1}{0} \\
b 0 \\
\nexists \\
\end{array}$ & $\begin{array}{l}\text { N } \\
\text { Oे } \\
\text { Un }\end{array}$ & $\begin{array}{l}\infty \\
\ddot{u} \\
\ddot{n}\end{array}$ & 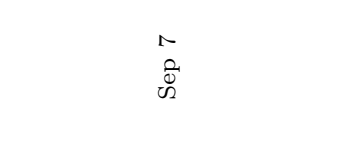 & $\begin{array}{l}\infty \\
\text { Qे } \\
\tilde{u}\end{array}$ & 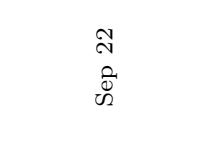 \\
\hline 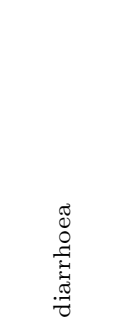 & 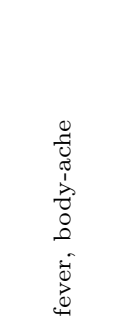 & 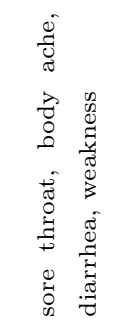 & 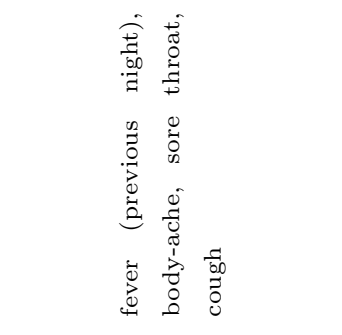 & 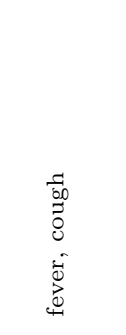 & 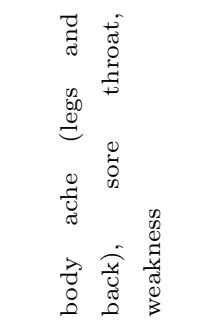 \\
\hline 紊 & 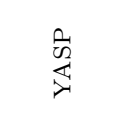 & $\sum_{\substack{n \\
\sum}}^{n}$ & $\sum_{>}^{0}$ & $\sum_{\lambda}^{0}$ & $\begin{array}{l}\sum_{i}^{2} \\
\sum_{i}^{+} \\
\sum_{n}\end{array}$ \\
\hline $\begin{array}{l}\vec{\nabla} \\
\text { مै }\end{array}$ & $\begin{array}{l}\text { N } \\
\text { षे } \\
\text { On }\end{array}$ & $\begin{array}{l}\overrightarrow{1} \\
\stackrel{\vec{v}}{\Omega}\end{array}$ & 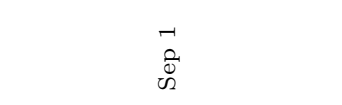 & $\begin{array}{l}\stackrel{\overrightarrow{0}}{ } \\
\hat{\mathrm{v}}\end{array}$ & 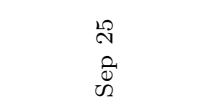 \\
\hline $\overrightarrow{a_{1}}$ & $\stackrel{N}{2}$ & $\stackrel{m}{\vec{a}}$ & $\vec{I}$ & $\stackrel{20}{a}$ & $\stackrel{0}{a}$ \\
\hline
\end{tabular}




\begin{tabular}{|c|c|c|c|c|c|}
\hline & 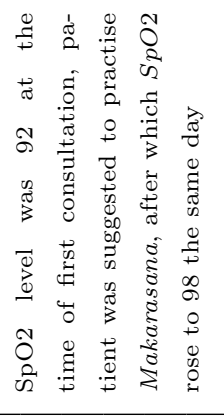 & & 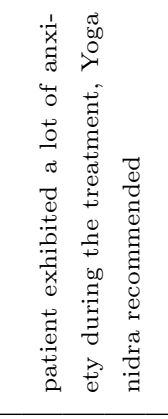 & 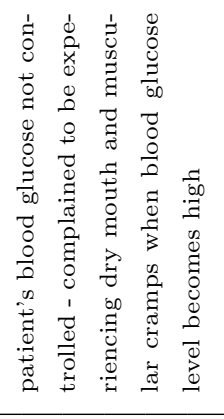 & 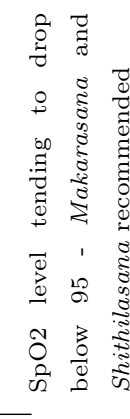 \\
\hline 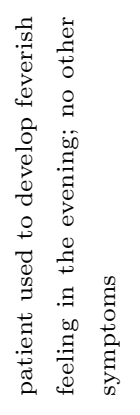 & 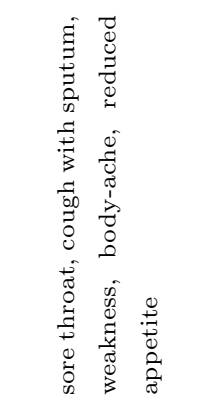 & 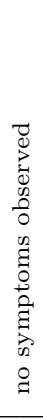 & 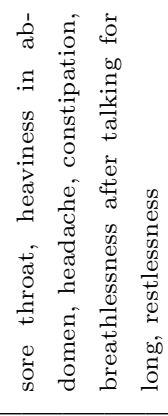 & 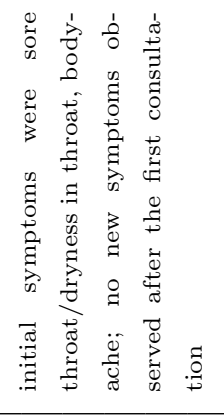 & 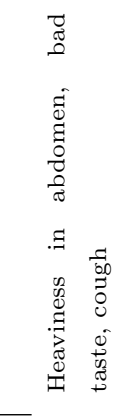 \\
\hline $\begin{array}{l}\vec{N} \\
\stackrel{े}{u} \\
\tilde{\omega}\end{array}$ & $\begin{array}{l}\infty \\
\stackrel{1}{ } \\
\stackrel{8}{0} \\
\circlearrowleft\end{array}$ & $\begin{array}{l}\text { N } \\
\stackrel{U}{U} \\
O\end{array}$ & $\begin{array}{l}= \\
\overrightarrow{0} \\
0\end{array}$ & $\stackrel{10}{\stackrel{10}{\breve{U}}}$ & $\begin{array}{l}\vec{N} \\
\vec{U} \\
0\end{array}$ \\
\hline 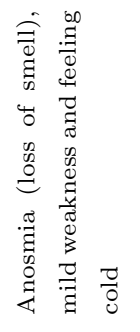 & 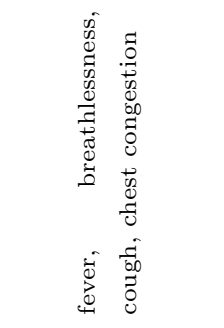 & 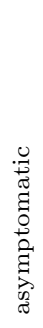 & 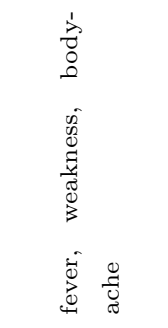 & 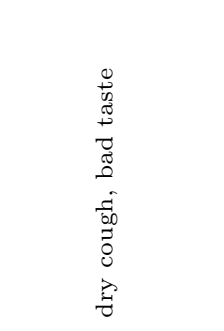 & 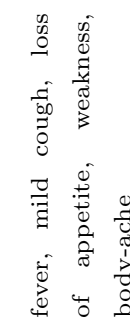 \\
\hline 至 & 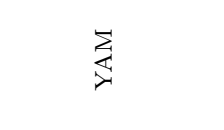 & 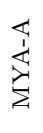 & $\sum_{i}$ & 梁 & $\sum_{\substack{n \\
\sum}}^{\infty}$ \\
\hline $\begin{array}{l}\stackrel{\infty}{N} \\
\stackrel{u}{u} \\
\tilde{\omega}\end{array}$ & $\begin{array}{l}\stackrel{\text { N }}{ } \\
\stackrel{े}{u} \\
\text { ñ }\end{array}$ & $\begin{array}{l}0 \\
\stackrel{艹}{0}\end{array}$ & $\begin{array}{l}\text { ㄱ. } \\
+ \\
\dot{U} \\
0\end{array}$ & $\begin{array}{l}\text { I } \\
\vec{U} \\
0\end{array}$ & $\begin{array}{l}\text { N } \\
+ \\
\tilde{U}\end{array}$ \\
\hline$\hat{\mathrm{O}}$ & $\stackrel{\infty}{2}$ & $\overrightarrow{\vec{\partial}}$ & ฌి & $\overrightarrow{\tilde{a}}$ & ָิ \\
\hline
\end{tabular}




\begin{tabular}{|c|c|c|c|}
\hline & & 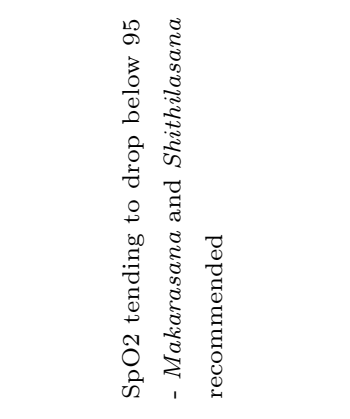 & 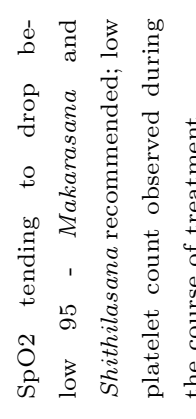 \\
\hline 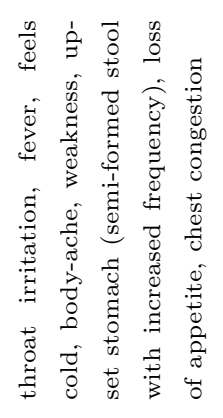 & 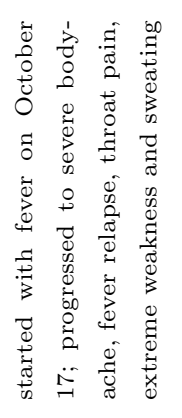 & 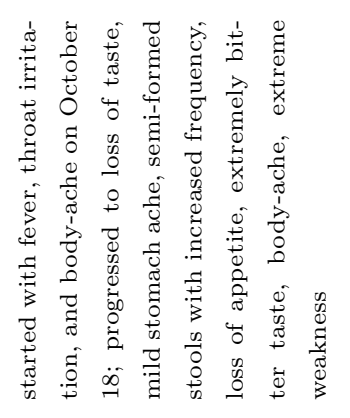 & 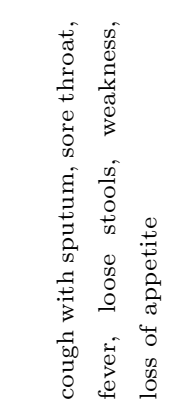 \\
\hline $\begin{array}{l}\stackrel{\infty}{:} \\
\stackrel{0}{0} \\
0\end{array}$ & $\begin{array}{l}\vec{N} \\
\vec{U} \\
0\end{array}$ & $\begin{array}{l}\vec{N} \\
\stackrel{U}{0}\end{array}$ & $\begin{array}{l}\mathcal{O} \\
\dot{z} \\
z \\
z\end{array}$ \\
\hline 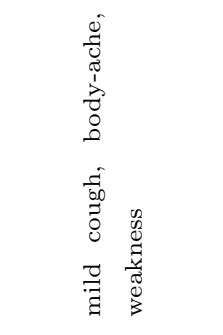 & 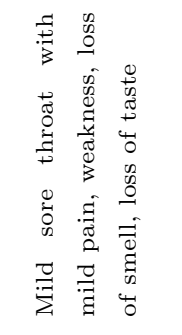 & 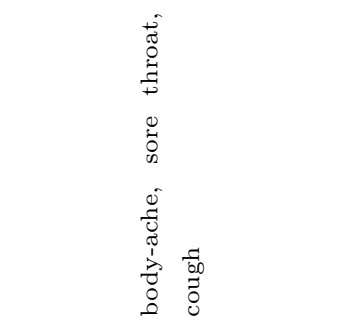 & 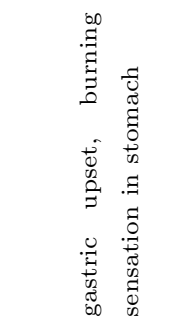 \\
\hline 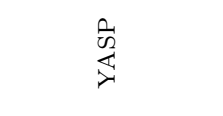 & $\sum_{\substack{i \\
\sum}}^{\infty}$ & 足 & 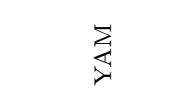 \\
\hline $\begin{array}{l}\vec{N} \\
\vec{U} \\
\dot{0}\end{array}$ & $\begin{array}{l}\vec{N} \\
\dot{U} \\
0\end{array}$ & $\begin{array}{l}\stackrel{i}{1} \\
\stackrel{U}{0} \\
0\end{array}$ & $\begin{array}{l}\stackrel{0}{N} \\
\dot{U} \\
0\end{array}$ \\
\hline$\stackrel{\Re}{\AA}$ & $\underset{\tilde{N}}{\stackrel{N}{ }}$ & $\stackrel{\mathscr{D}}{\stackrel{2}{\alpha}}$ & $\stackrel{\stackrel{\leftrightarrow}{L}}{ }$ \\
\hline
\end{tabular}




\begin{tabular}{|c|c|c|c|}
\hline 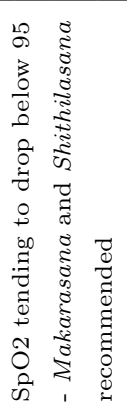 & & & \\
\hline 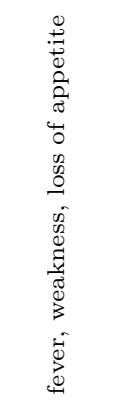 & 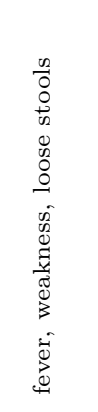 & 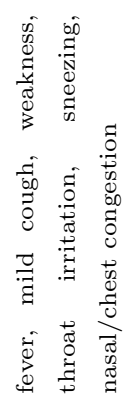 & 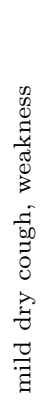 \\
\hline $\begin{array}{l}\infty \\
8 \\
b \\
z \\
z\end{array}$ & $\begin{array}{l}8 \\
8 \\
\vdots \\
z\end{array}$ & $\begin{array}{l}5 \\
\Delta \\
0 \\
z\end{array}$ & $\begin{array}{l}\hat{o} \\
b \\
\vdots \\
z\end{array}$ \\
\hline 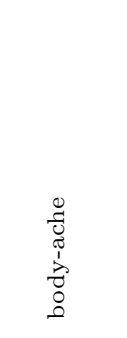 & 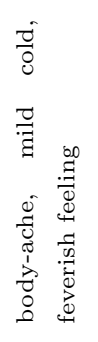 & 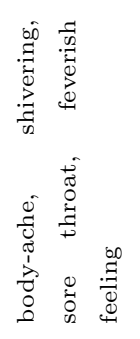 & 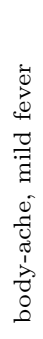 \\
\hline 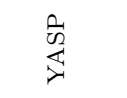 & 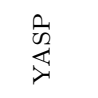 & 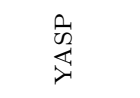 & $\sum_{i}^{0}$ \\
\hline $\begin{array}{l}\stackrel{\infty}{N} \\
\stackrel{u}{u} \\
0\end{array}$ & $\begin{array}{l}\overrightarrow{0} \\
\vec{b} \\
z\end{array}$ & $\begin{array}{l}\mathfrak{1} \\
\vdots \\
b \\
z\end{array}$ & $\begin{array}{l}8 \\
8 \\
b \\
z\end{array}$ \\
\hline$\stackrel{\hat{\Lambda}}{\hat{n}}$ & $\stackrel{\infty}{\stackrel{\infty}{\varpi}}$ & ๙ิ & $\stackrel{\circ}{\infty}$ \\
\hline
\end{tabular}




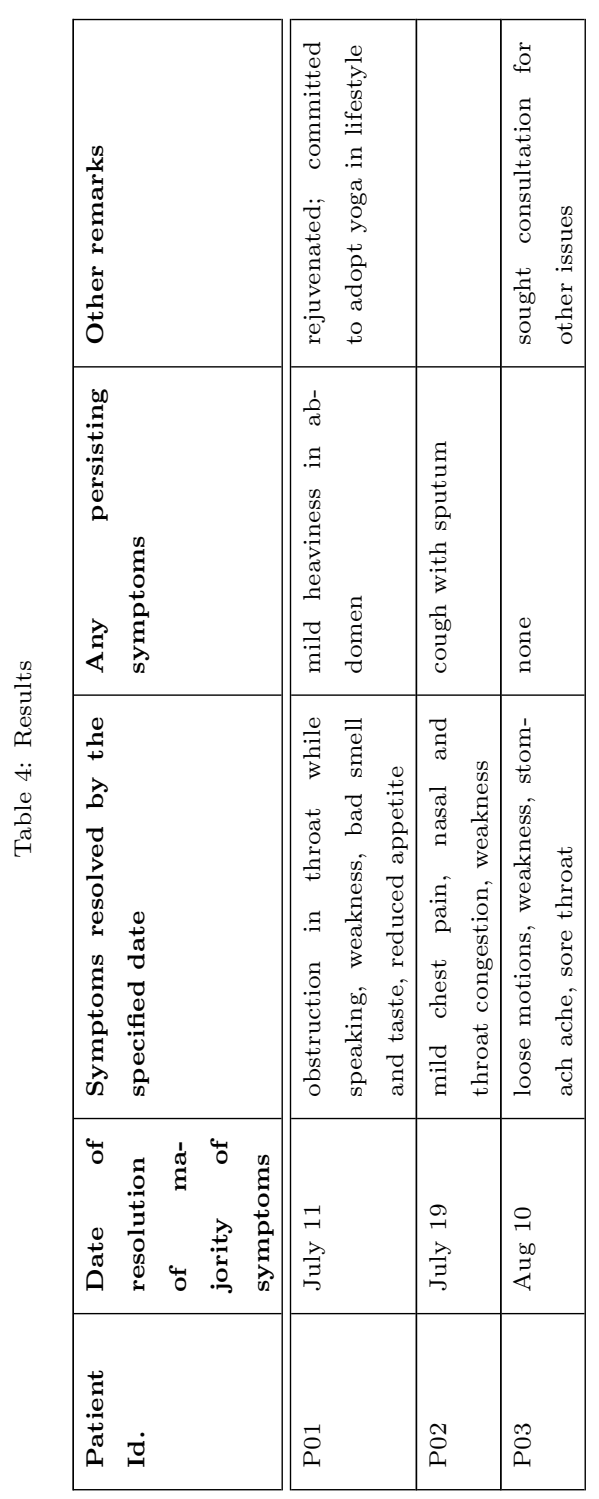




\begin{tabular}{|c|c|c|c|}
\hline 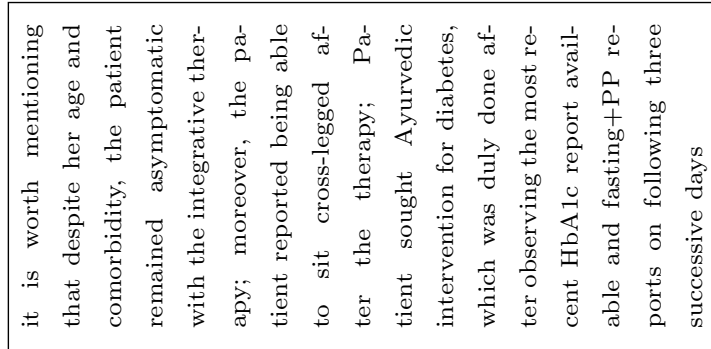 & & & 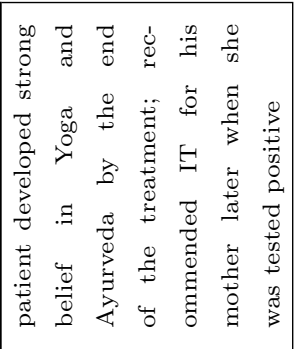 \\
\hline 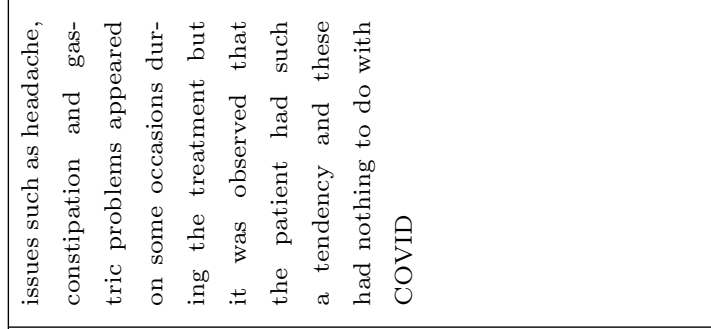 & 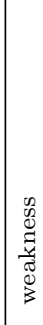 & 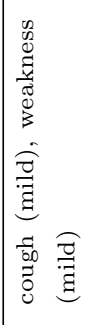 & 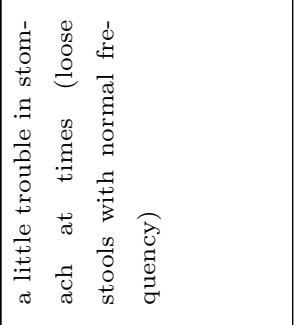 \\
\hline 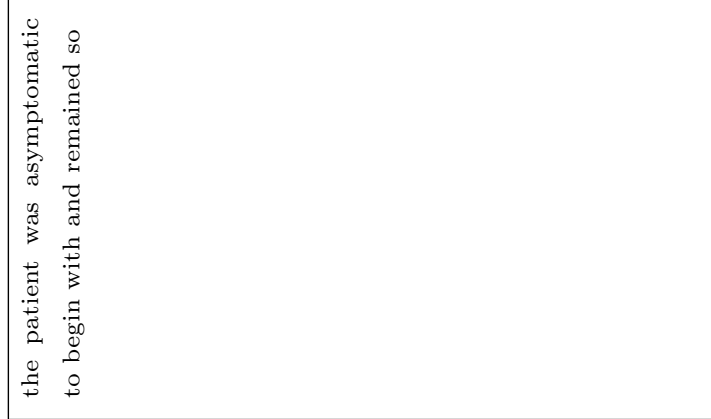 & 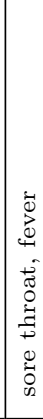 & 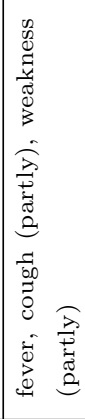 & 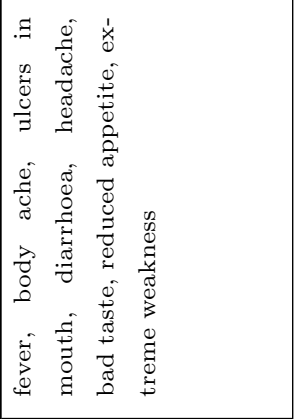 \\
\hline 岁 & 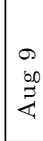 & & $\begin{array}{l}\vec{y} \\
\stackrel{8}{*}\end{array}$ \\
\hline 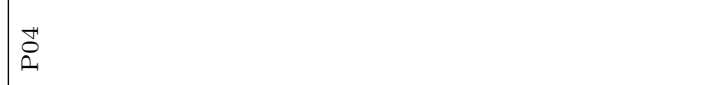 & 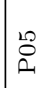 & 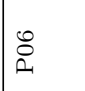 & 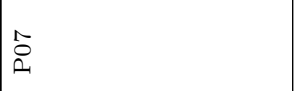 \\
\hline
\end{tabular}




\begin{tabular}{|c|c|c|c|c|c|}
\hline 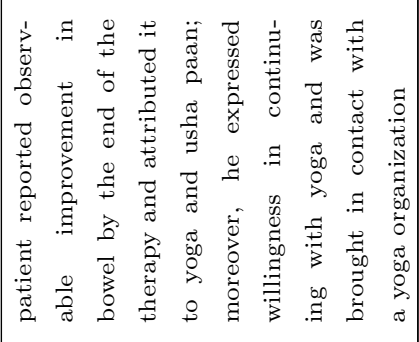 & & & 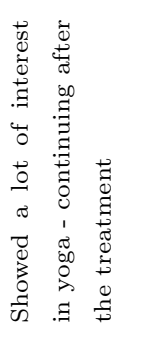 & & \\
\hline 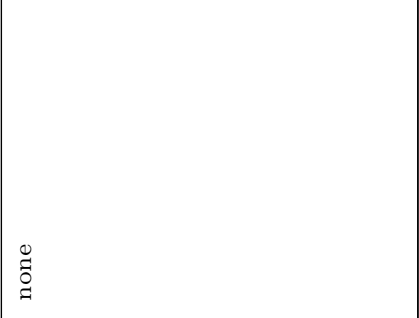 & 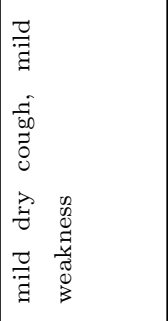 & : & 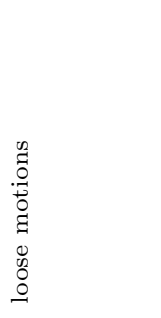 & 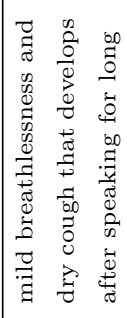 & 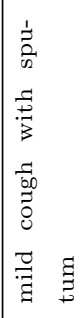 \\
\hline $\bar{\sigma}$ & 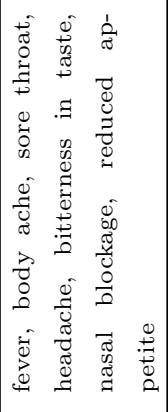 & 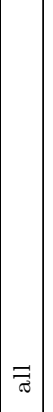 & & 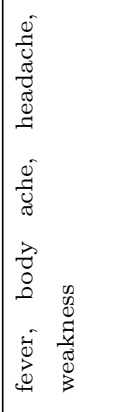 & 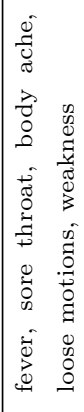 \\
\hline 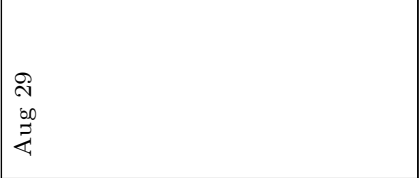 & $\begin{array}{l}0 \\
\stackrel{1}{0} \\
\stackrel{\otimes}{u} \\
\tilde{u}\end{array}$ & 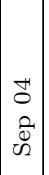 & 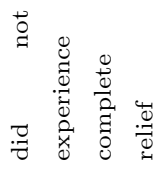 & 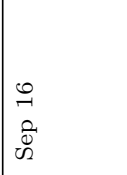 & 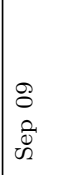 \\
\hline 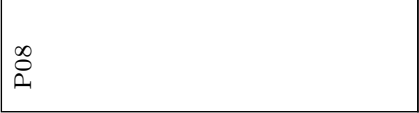 & $\stackrel{8}{2}$ & $\stackrel{\circ}{a}$ & $\vec{a}$ & $\frac{\vec{a}}{\vec{D}}$ & $\stackrel{m}{\vec{L}}$ \\
\hline
\end{tabular}




\begin{tabular}{|c|c|c|c|c|}
\hline 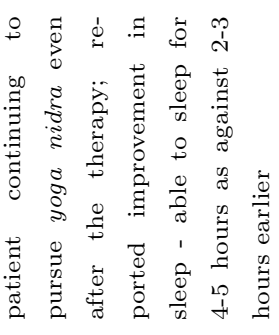 & 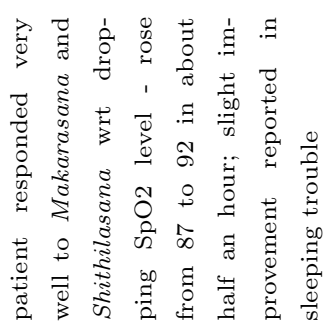 & & 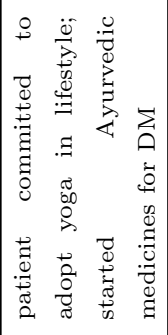 & \\
\hline : & 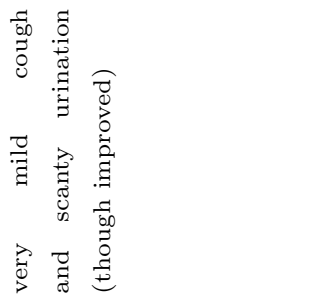 & 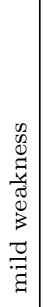 & $\begin{array}{l}\Xi \\
0 \\
\Xi\end{array}$ & $\begin{array}{l}\frac{\pi}{80} \\
\overrightarrow{0} \\
\dot{0} \\
\overrightarrow{0} \\
\vec{\Xi}\end{array}$ \\
\hline సี & & 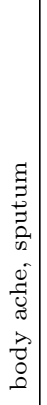 & & 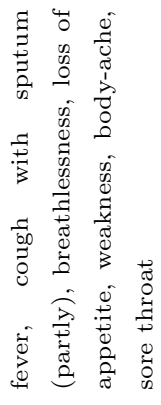 \\
\hline $\begin{array}{l}\overrightarrow{7} \\
\text { Oे. } \\
\text { vin }\end{array}$ & 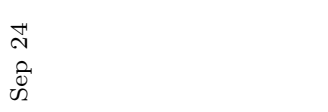 & $\begin{array}{l}\tilde{D} \\
\vec{u} \\
0\end{array}$ & & $\begin{array}{l}8 \\
\ddot{U} \\
0\end{array}$ \\
\hline$\underset{\Delta}{\vec{\Delta}}$ & $\vec{\sim}$ & $\overrightarrow{2}$ & $\frac{1}{2}$ & $\stackrel{\infty}{\tilde{z}}$ \\
\hline
\end{tabular}




\begin{tabular}{|c|c|c|c|}
\hline 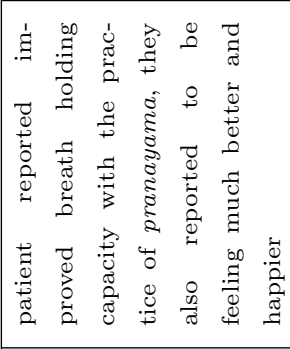 & 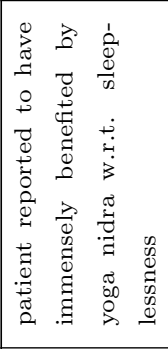 & 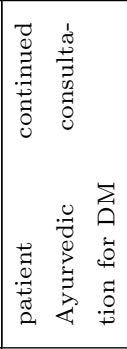 & 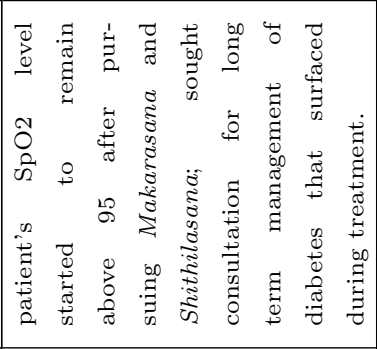 \\
\hline 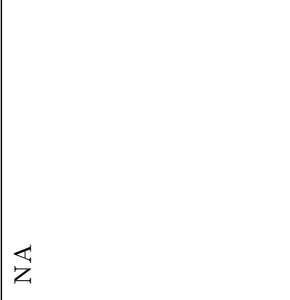 & 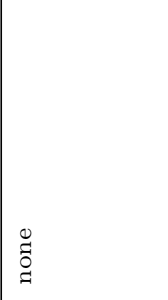 & $\begin{array}{l}\stackrel{\Xi}{0} \\
\stackrel{a}{2}\end{array}$ & $\begin{array}{l}\stackrel{0}{0} \\
\Xi\end{array}$ \\
\hline$\overleftrightarrow{z}$ & 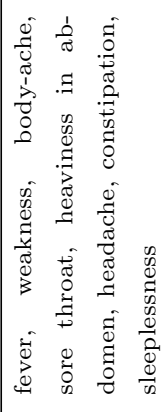 & $\bar{\sigma}$ & 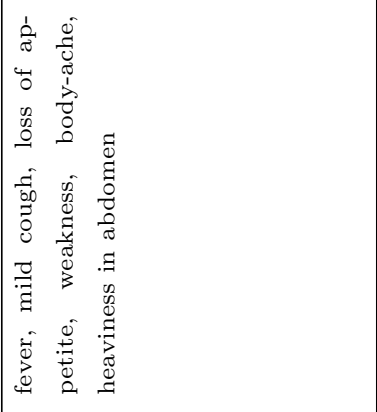 \\
\hline$\overleftrightarrow{\mathrm{z}}$ & $\begin{array}{l}\text { N } \\
\stackrel{U}{0} \\
0\end{array}$ & $\begin{array}{l}\mathcal{N} \\
\stackrel{U}{0}\end{array}$ & \\
\hline $\overrightarrow{\vec{m}}$ & $\stackrel{\stackrel{N}{~}}{ }$ & $\overrightarrow{\hat{\aleph}}$ & 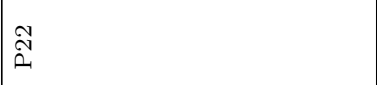 \\
\hline
\end{tabular}




\begin{tabular}{|c|c|c|c|}
\hline & & & 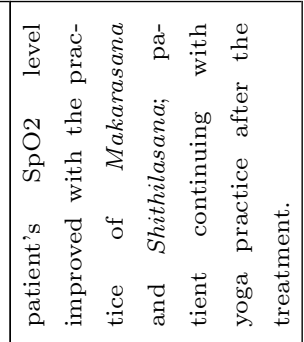 \\
\hline 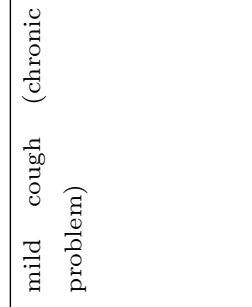 & 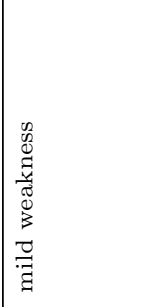 & 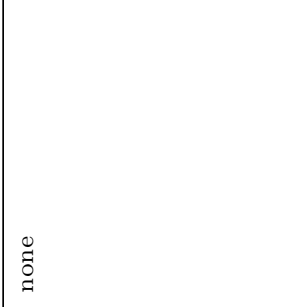 & 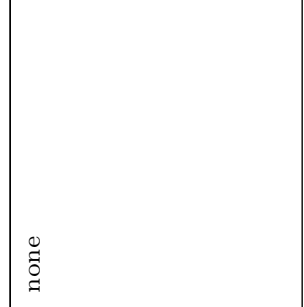 \\
\hline 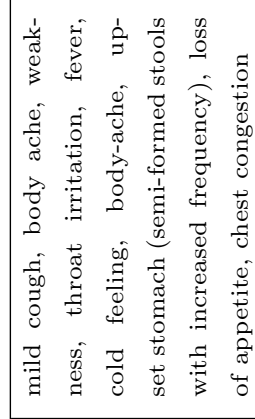 & 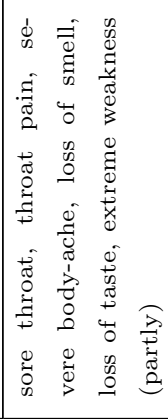 & 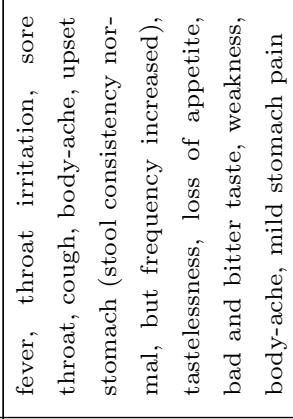 & 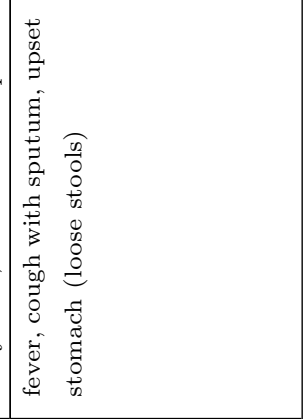 \\
\hline $\begin{array}{l}\overrightarrow{0} \\
\vec{b} \\
z\end{array}$ & & & $\begin{array}{l}\mathcal{N} \\
\mathcal{1} \\
z \\
z\end{array}$ \\
\hline 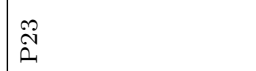 & 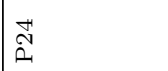 & 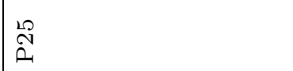 & 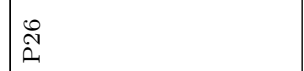 \\
\hline
\end{tabular}




\begin{tabular}{|c|c|c|c|}
\hline 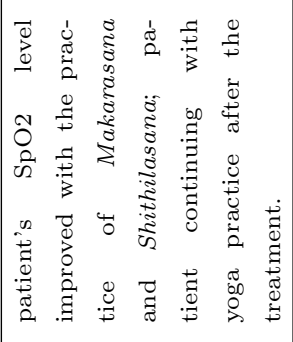 & & & 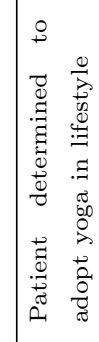 \\
\hline : & : & 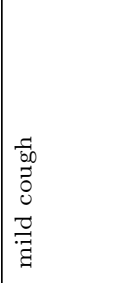 & $\begin{array}{l}\mathscr{\Xi} \\
\stackrel{g}{0}\end{array}$ \\
\hline 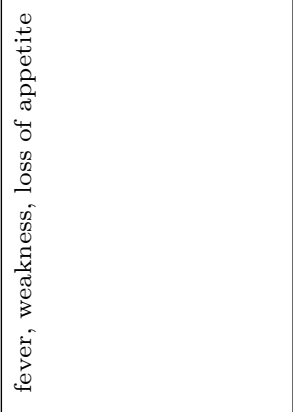 & 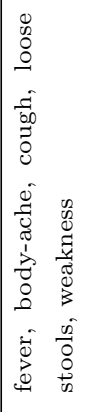 & 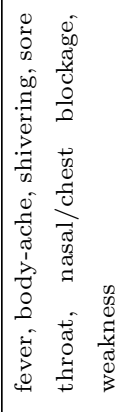 & 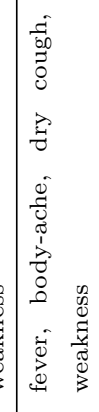 \\
\hline 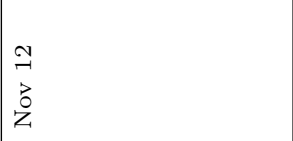 & & & 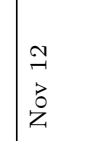 \\
\hline$\tilde{E}$ & 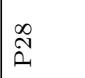 & ڤે & $\stackrel{\infty}{\infty}$ \\
\hline
\end{tabular}




\section{Supplementary Material}

800

Here we furnish details about the progress during the course of treatment for each patient. It may be noted that though some patients started their treatment with modern western medicine, the presented case series pertains to Ayurvedaand-Yoga-based treatment. Thus, the days referred to in the detailed presenta-

\section{Detailed history, treatment plan and progress tracking for P01:}

The patient presented with the following symptoms at the time of first consulta820 (as reported, the patient used to get exhausted even on doing his daily chores); heaviness in the abdomen (gastric upset); frequent bloating feeling in the stomach; bad smell and taste, reduced appetite. Moreover, he sounded very low and lacking in energy. asymptomatic three weeks prior to the first consultation. Later, he developed fever and symptoms of pneumonia, following which he went to the hospital and underwent the RT-PCR test. He reported to have had fever for about 10 days 
until June 22, 2020. The patient was detected positive on June 24, 2020. Subsequently, he was prescribed the following allopathic medication for 10 days - Nizonide 500mg (Nitazoxanide 500 mg), Doxy 100mg (Doxycycline 100mg), PAN 40mg (Pantoprazole 40mg), Vitamin C, Matilda-ER (Methylcobalamin, Pyridoxine and Folic acid), LCZ 5mg (Levocetirizine 5mg). On July 1, 2020, at the time of the first consultation for the Integrative Therapy, the patient was home quarantined. He also reported to have had dry cough and body-ache earlier, both of which no longer persisted at the time of his first consultation. The following allopathic medicines were being taken regularly by the patient for DM and Hypothyroidism: Glimepiride-2mg, Galvas-50mg, Vobit-0.3mg, Torcilin-10, Thyroxin $75 \mathrm{mcg}$. Despite taking allopathic medicines, the patient's blood sugar levels were generally as follows: FBS > $200 \mathrm{mg} / \mathrm{dl}$, PPBS $>250 \mathrm{mg} / \mathrm{dl}$; PPBS was reported to be $230 \mathrm{mg} / \mathrm{dl}$ on June 30 .

Based on the symptoms at the time of first consultation and the patient's medical history, he following prescription for the Integrative Therapy was given: Ayurvedic medicines and procedures prescribed: Giloy Ghanvati (3 doses of 2 tablets each to be taken after meal with lukewarm water), Ashwagandha vati (2 doses of 2 tablets each to be taken after meal), Pathyadi Kwath - pravahi (15 ml mixed with an equal quantity of lukewarm water to be consumed twice a day 30 minutes after meal), and Diabecon (2 doses of 1 tablet each to be taken 30 minutes before meal).

850 The Yoga protocol designed for the patient comprised Sukshma vyayama (upper and lower body parts), breathing exercises (4 exercises with 5 iterations in each), asanas (parshva sukhasana, sukhasana twist, utthana mandukasana, ardha ushtrasana, meru vakrasana, ardha halasana with one leg folded, anantasana, pawan muktasana); Shavasana, Pranayama (sectional breathing and 855 full yogic breathing, anulom-vilom, bhramari, udgeet), and Dhyana. Looking at the anxiety level of the patient, he was also suggested to practise Yoga nidra [56] (guided Yogic sleep and relaxation practice), for which he was provided a link to guided audio instructions for Yoga nidra (in Hindi language) by Swami Satyananda Saraswati [52], the duration of the practice being 42 minutes. 
The patient complied to the prescribed medication and suggested procedures almost fully; he was also very regular with the yoga sessions. Moreover, he adopted many modifications and yoga in is lifestyle. During follow-up, he several times remarked that he could feel many changes in his overall health in such a short span of time.

865

Details of the patient's progress and compliance during the course of the treatment are given below in Table 5 . More details about this case may be found in the case report [44].

\begin{tabular}{|c|c|c|c|}
\hline Day & Date & Vital Measures & General Remarks \\
\hline 0 & July 1 & & $\begin{array}{l}\text { Symptoms reported/ observed: occasional ob- } \\
\text { struction in throat while speaking for long; ex- } \\
\text { treme weakness (gets exhausted even on doing } \\
\text { his own daily chores); sounded very low and } \\
\text { lacking in energy; heaviness in the abdomen } \\
\text { (gastric upset); frequent bloating feeling in the } \\
\text { stomach; bad smell and taste (occurred less fre- } \\
\text { quently than it did earlier), reduced appetite; } \\
\text { patient also exhibited disease-induced anxiety. }\end{array}$ \\
\hline 1 & July 2 & & $\begin{array}{l}\text { Most symptoms from the previous day persist; } \\
\text { patient reported uneasiness in body. }\end{array}$ \\
\hline 2 & July 3 & FBS: 169 & $\begin{array}{l}\text { Improvement observed; approximately } 25 \% \text { re- } \\
\text { lief reported in all the symptoms; lightness in } \\
\text { the body reported. }\end{array}$ \\
\hline 3 & July 4 & FBS: 190, PPBS: 206 & $\begin{array}{l}\text { Doing Yoga twice daily; feeling good, with im- } \\
\text { provement in all the symptoms. }\end{array}$ \\
\hline 4 & July 5 & FBS: 160, PPBS: 206 & $\begin{array}{l}\text { Approximately } 50 \% \text { relief reported in all the } \\
\text { symptoms; lightness in the body reported. }\end{array}$ \\
\hline 5 & July 6 & FBS: 155 , PPBS: 175 & $\begin{array}{l}\text { No weakness; normal appetite; taste and smell } \\
\text { almost normal; approximately } 75 \% \text { relief re- } \\
\text { ported. }\end{array}$ \\
\hline 6 & July 7 & FBS: 140, Random: 110 & $\begin{array}{l}\text { No sore throat; no weakness; normal taste and } \\
\text { smell; almost no other problem except mild } \\
\text { heaviness in abdomen; approximately } 80 \% \text { re- } \\
\text { lief reported. }\end{array}$ \\
\hline
\end{tabular}




\begin{tabular}{|c|c|c|c|}
\hline 7 & July 8 & FBS: 140, Random: 110 & No problem except mild heaviness in abdomen. \\
\hline 8 & July 9 & FBS: 130, PPBS: 230 & $\begin{array}{l}\text { Second RT-PCR report received on July } 8 \text { was } \\
\text { +ve, patient had anxiety again; slight change } \\
\text { done in the prescription: i) Diabecon - } 1 \text { tab. } \\
\text { in the morning and } 2 \text { tab. in the evening (ear- } \\
\text { lier it was } 1 \text { tab. in the evening) ii) advised to } \\
\text { take (ajwain + saunf + dhaniya) powder - } 1 / 2 \\
\text { spoon, with lukewarm water, after meal. }\end{array}$ \\
\hline 9 & July 10 & FBS: 110, PPBS: 150 & $\begin{array}{l}\text { Improvement in all the problems (including } \\
\text { heaviness in abdomen). }\end{array}$ \\
\hline 10 & July 11 & FBS: 110, PPBS: 150 & $\begin{array}{l}\text { No problem except mild heaviness in abdomen, } \\
\text { improvement in anxiety; dose of Diabecon } \\
\text { changed to } 2 \text { tab. BD (patient advised to start } \\
\text { the revised dose from July 12). }\end{array}$ \\
\hline 11 & July 12 & FBS: 130, PPBS: 140 & $\begin{array}{l}\text { No problem except mild heaviness in abdomen; } \\
\text { started Diabecon } 2 \text { tab. BD. }\end{array}$ \\
\hline 12 & July 13 & FBS: 99, PPBS: 112 & No problem except mild heaviness in abdomen. \\
\hline 13 & July 14 & FBS: 108, PPBS: 112 & $\begin{array}{l}\text { Sample given for RT-PCR test; no problem ex- } \\
\text { cept mild heaviness in abdomen. }\end{array}$ \\
\hline 14 & July 15 & FBS: 120, PPBS: 165 & No problem except mild heaviness in abdomen. \\
\hline 15 & July 16 & FBS: 108, PPBS: 140 & $\begin{array}{l}\text { RT-PCR test inconclusive; no problem except } \\
\text { mild heaviness in abdomen. }\end{array}$ \\
\hline 16 & July 17 & FBS: 90, PPBS: 140 & No problem except mild heaviness in abdomen. \\
\hline 17 & July 18 & & $\begin{array}{l}\text { No problem except mild heaviness in abdomen; } \\
\text { no more anxiety. }\end{array}$ \\
\hline 18 & July 19 & & No problem except mild heaviness in abdomen. \\
\hline 19 & July 20 & & $\begin{array}{l}\text { Sample given for RT-PCR test; the patient's al- } \\
\text { lopathic doctor reduced the dosage of his dia- } \\
\text { betes medication, i.e. Glimepiride changed from } \\
2 \mathrm{mg} \text { to } 1 \mathrm{mg} \text {; no problem except mild heaviness } \\
\text { in abdomen. }\end{array}$ \\
\hline
\end{tabular}




\begin{tabular}{|c|c|l|l|}
\hline 20 & July 21 & & $\begin{array}{l}\text { Diabecon changed to Diabecon DS 1 tab. BD; } \\
\text { no problem except mild heaviness in abdomen. }\end{array}$ \\
\hline 21 & July 22 & & $\begin{array}{l}\text { RT-PCR reported no infection (negative); } \\
\text { no problem except mild heaviness in abdomen. }\end{array}$ \\
\hline
\end{tabular}

Table 5: Progress of patient P01 and evolution of the treatment plan

\section{Detailed history, treatment plan and progress tracking for P02:}

870 The patient presented with the following symptoms at the time of first consultation: cough with sputum, mild chest pain, nasal and throat congestion and mild weakness.

History of the present illness was taken, which is as follows. The patient had developed fever some time back that persisted for 2-3 days, following which he underwent the RT-PCR test on July 4. The result of the test (shared with him on July 8) confirmed that he was COVID positive. Subsequently, he was prescribed the following allopathic medication - Vit D, Becasule, Bro-Zedex syrup, Novaclav 625. On July 9, 2020, at the time of the first consultation, the patient had been taking these medicines. Besides, the patient had been taking homemade kadha, drinking warm water and doing gargles.

The following allopathic medicines were being taken regularly by the patient for DM and Hypothyroidism: Gemer P1, Thyronorm 25 mcg. Despite taking allopathic medicines, the patient's blood sugar levels were observed as follows on April 22: FBS $118 \mathrm{mg} / \mathrm{dl}$, PPBS $247 \mathrm{mg} / \mathrm{dl}$; his TSH was found to be 5.6 on ${ }_{885}$ April 22. The patient also reported that his SpO2 level remained around 92-93. Based on the symptoms at the time of first consultation and the patient's medical history, he following prescription for the Integrative Therapy was given: Ayurvedic medicines and procedures prescribed: Lavangadi vati (2 doses of 2 tablets each to be sucked after meal), Giloy Ghanvati (3 doses of 2 tablets each to be taken after meal with lukewarm water), Pathyadi Kwath - pravahi (15 ml mixed with an equal quantity of lukewarm water to be consumed twice a day 30 minutes after meal), and Diabecon ( 2 doses of 1 tablet each to be taken 30 
minutes before meal), nasya (putting 2 drops of oil in each nostril) to be done twice a day (morning and evening) with mustard oil; steam inhalation and gargles with medicated water containing ajowan caraway, rock salt and turmeric. Yoga Protocol: Sukshma Vyayama (upper and lower body parts); Breathing exercises (4 exercises with 5 iterations in each); Asanas: (tadasana, triyaktadasana, trikonasana, hastapadasana, ardhachakrasana, utthana mandukasana, vakrasana, bhujangasana, shalabhasana, ardhahalasana, matsyasana); Shavasana; Pranayama (anulom-vilom, sectional breathing and full yogic breathing, bhramari, udget); Dhyana

The patient was only partially compliant with respect to the ayurvedic medicines, but very regular and passionate about yoga sessions. He expressed his wish to continue the ongoing allopathic medication for DM, instead of taking Diabecon. Besides, he was reluctant to take Lavangadi vati; instead he started Kantha Sudharak vati on his own. However, he was regular in taking all other medicines and following the prescribed procedures; also, he continued to practise yoga with the recorded video provided to him for self-practice.

Details of the patient's progress and compliance during the course of the treatment are given below in Table 6 .

\begin{tabular}{|c|c|c|c|}
\hline Day & Date & Vital Measures & General Remarks \\
\hline 0 & July 9 & SpO2: $92-93$ & $\begin{array}{l}\text { Symptoms observed/reported: cough with spu- } \\
\text { tum, chest pain (mild), nasal and throat con- } \\
\text { gestion, weakness (mild) }\end{array}$ \\
\hline 2 & July 11 & & Patient remained non-compliant till this day. \\
\hline 3 & July 12 & & $\begin{array}{l}\text { Patient started the prescribed medication par- } \\
\text { tially; vitals not shared }\end{array}$ \\
\hline 5 & July 14 & & $\begin{array}{l}\text { Patient reported about } 20 \% \text { improvement in all } \\
\text { the symptoms; yoga sessions commenced }\end{array}$ \\
\hline 8 & July 17 & $\begin{array}{l}\text { SpO2: } 95-96 \\
\text { Temp: } 96.6-97.5\end{array}$ & $\begin{array}{l}\text { Patient reported about } 50 \% \text { improvement in all } \\
\text { the symptoms; feeling light; } \mathrm{SpO} 2 \text { reported to } \\
\text { fluctuate between } 92 \text { and } 96\end{array}$ \\
\hline
\end{tabular}




\begin{tabular}{|c|l|l|l|}
\hline 9 & July 18 & $\begin{array}{l}\text { SpO2: 96-97 } \\
\text { Temp: 97.1-97.6 }\end{array}$ & \\
\hline 10 & July 19 & & $\begin{array}{l}\text { Patient reported about 90\% improvement; } \\
\text { only symptom persisting was cough with spu- } \\
\text { tum; advised to do kunjal twice weekly. }\end{array}$ \\
\hline 11 & July 20 & SpO2: $96 \quad$ Temp: 97.6 & \\
\hline
\end{tabular}

Table 6: Progress of patient P02 and evolution of the treatment plan

\section{Detailed treatment plan and progress tracking for P03:}

The patient presented with the following symptoms at the time of first consultation: sore throat (mild), stomach ache (mild), weakness (mild), loose motions. History of the present illness was taken, which is as follows. The patient also reported having tendency of watery stool (with normal frequency) since about two and a half months. The patient, after being tested positive for COVID on July 26, had the following vital measurements - BP: 187/127; PR: 94; SpO2: 98; Blood glucose FBS: 110-115, Random: 112. Due to high blood pressure, he was temporarily hospitalized. However, the BP came down to 150/93 within half an hour, following which he was given the option of being home quarantined, which the patient opted for. Thus, he was discharged within 2 hours of admission and was prescribed the following allopathic medicines: Vitamin C, Zinc, Vitamin E (Evion $400 \mathrm{mg}$ ), Azithromycin.

${ }_{925}$ The following allopathic medicines were being taken regularly by the patient: Galvas Met 50mg/500mg (for DMT2), Olmetrack 20 (for HTN). He informed that his blood glucose level usually remained under control with his ongoing medication, and therefore he did not require any extra medicines for the same (however, his HbA1c report dated May 27, 2020 showed the value of 7.1\%, which 930 is above normal). As reported by the patient, the blood glucose level (fasting) remained controlled (between 110 and $115 \mathrm{mg} / \mathrm{dl}$ ). As observed in the daily vitals shared by the patient (starting July 26 upto the time of first consultation on July 30), the blood pressure remained controlled (systolic mostly between 
126 and 140 and diastolic mostly between 77 and 86.

Based on the symptoms at the time of first consultation and the patient's medical history, he following prescription for the Integrative Therapy was given: Ayurvedic medicines and procedures prescribed: Sanshamani vati (3 doses of 2 tablets each to be taken after meal), Ashwagandha vati, Pathyadi Kwath pravahi $(15 \mathrm{ml}$ mixed with an equal quantity of lukewarm water to be consumed twice a day empty stomach), nasya (putting 2 drops of oil in each nostril) to be done twice a day (morning and evening) with anu teil.

Yoga Protocol: Sukshma Vyayama (upper and lower body parts); Breathing exercises (4 exercises with 5 iterations in each); Asanas: (tadasana, trikonasana, hastapadasana, ardhachakrasana, parshva sukhasana, sukhasana twist, utthana mandukasana, bhujangasana, shalabhasana, ardhahalasana with one leg folded, pawanmuktasana); Shavasana; Pranayama (sectional breathing and full yogic breathing, bhramari, anulom-vilom, udgeet); Dhyana

The patient was almost fully compliant with respect to Ayurvedic medicines as well as yoga sessions. He regularly took medicines and attended most of the yoga sessions. By the end of the treatment, he was convinced about the therapy by the benefits he had observed. Therefore, he sought advice for other long-term medical issues not related to COVID. The same was duly addressed.

Details of the patient's progress and compliance during the course of the treat955 ment are given below in Table 7 .

\begin{tabular}{|c|c|c|c|c|}
\hline Day & Date & Vital Measures & & General Remarks \\
\hline 0 & July 30 & $\begin{array}{l}\text { SpO2: } 97-98 \\
\text { BP sys: } 125-144 \\
\text { Temp: } 97.3-98.1\end{array}$ & $\begin{array}{l}\text { PR: } 76-88 \\
\text { dias: } 70-90\end{array}$ & $\begin{array}{l}\text { Symptoms reported/ ob- } \\
\text { served: sore throat (mild), } \\
\text { stomach ache (mild), weak- } \\
\text { ness (mild), loose motions. }\end{array}$ \\
\hline 1 & July 31 & $\begin{array}{l}\text { SpO2: } 98 \\
\text { BP: } 136 / 69\end{array}$ & $\begin{array}{l}\text { PR: } 75 \\
\text { Temp: } 97.2\end{array}$ & \\
\hline 2 & Aug 1 & $\begin{array}{l}\text { SpO2: } 95-98 \\
\text { BP sys: } 130-144 \\
\text { Temp: } 97.5-97.8\end{array}$ & $\begin{array}{l}\text { PR: } 80-98 \\
\text { dias: } 81-90 \\
\quad 56\end{array}$ & $\begin{array}{l}\text { Patient complained of weak- } \\
\text { ness, dropping SpO2 and con- } \\
\text { stipation (also said consti- } \\
\text { pation was chronic) - Soft- } \\
\text { vac advised for constipation; } \\
\text { Makarsana and Shithilasana } \\
\text { for SpO2. }\end{array}$ \\
\hline
\end{tabular}




\begin{tabular}{|c|c|c|c|c|}
\hline 3 & Aug 2 & $\begin{array}{l}\text { SpO2: } 97-98 \\
\text { BP sys: } 141-160 \\
\text { Temp: } 97.6-98.2\end{array}$ & $\begin{array}{l}\text { PR: } 80-85 \\
\text { dias: } 86-96\end{array}$ & $\begin{array}{l}\text { Patient reported stable condi- } \\
\text { tion. }\end{array}$ \\
\hline 4 & Aug 3 & $\begin{array}{l}\text { SpO2: } 97-98 \\
\text { BP sys: } 138-151 \\
\text { Temp: } 97.7-98.4\end{array}$ & $\begin{array}{l}\text { PR: } 80-87 \\
\text { dias: } 90-93\end{array}$ & $\begin{array}{l}\text { Patient reported continuing } \\
\text { relief. }\end{array}$ \\
\hline 5 & Aug 4 & $\begin{array}{l}\text { SpO2: } 98-99 \\
\text { BP sys: } 140-145 \\
\text { Temp: } 97.7-98.5\end{array}$ & $\begin{array}{l}\text { PR:78-95 } \\
\text { dias: } 83-91 \\
\text { FBS: } 125\end{array}$ & $\begin{array}{l}\text { Patient reported continuing } \\
\text { relief. }\end{array}$ \\
\hline 6 & Aug 5 & $\begin{array}{l}\text { SpO2: } 97-99 \\
\text { BP sys: } 138-154 \\
\text { Temp: } 97.6-98.1\end{array}$ & $\begin{array}{l}\text { PR: } 79-90 \\
\text { dias: } 84-95\end{array}$ & $\begin{array}{l}\text { Patient's condition was found } \\
\text { stable and he reported contin- } \\
\text { uing relief. }\end{array}$ \\
\hline 7 & Aug 6 & $\begin{array}{l}\text { SpO2: } 98 \\
\text { BP sys: } 141-148 \\
\text { Temp: } 97.9-98.2\end{array}$ & $\begin{array}{l}\text { PR: } 80-90 \\
\text { dias: } 89-91\end{array}$ & $\begin{array}{l}\text { Patient's HbA1c report re- } \\
\text { ceived (dated Aug. 5) with } \\
\text { value } 7.3 \text {. }\end{array}$ \\
\hline 8 & Aug 7 & $\begin{array}{l}\text { SpO2: } 97-98 \\
\text { BP sys: } 133-141 \\
\text { Temp: } 98.0-98.4 \\
\text { FBS: } 125\end{array}$ & $\begin{array}{l}\text { PR: } 83-95 \\
\text { dias: } 81-87 \\
\text { PPBS: } 159\end{array}$ & $\begin{array}{l}\text { Patient reported to be feeling } \\
\text { better. }\end{array}$ \\
\hline 9 & Aug 8 & $\begin{array}{l}\text { SpO2: } 97-98 \\
\text { BP sys: } 135-144 \\
\text { Temp: } 98.2-98.8 \\
\text { FBS: } 111\end{array}$ & $\begin{array}{l}\text { PR: } 85-90 \\
\text { dias: } 84-91 \\
\text { PPBS: } 182\end{array}$ & $\begin{array}{l}\text { Patient progressively experi- } \\
\text { encing relief. }\end{array}$ \\
\hline 10 & Aug 9 & $\begin{array}{l}\text { SpO2: } 98-99 \\
\text { BP sys: } 139-143 \\
\text { Temp: } 97.7-98.1 \\
\text { FBS: } 100\end{array}$ & $\begin{array}{l}\text { PR: } 80-95 \\
\text { dias: } 79-86 \\
\text { PPBS: } 184\end{array}$ & Same as the previous day. \\
\hline 11 & Aug 10 & $\begin{array}{l}\text { SpO2: } 97-98 \\
\text { BP sys: } 125-139 \\
\text { Temp: } 97.7-97.8 \\
\text { FBS: } 112\end{array}$ & $\begin{array}{l}\text { PR: } 77-88 \\
\text { dias: } 77-87 \\
\text { PPBS: } 131\end{array}$ & $\begin{array}{l}\text { Patient reported complete re- } \\
\text { lief, including constipation. }\end{array}$ \\
\hline
\end{tabular}




\begin{tabular}{|c|c|c|c|c|}
\hline 12 & Aug 11 & $\begin{array}{l}\text { SpO2: } 98-99 \\
\text { BP sys: } 123-125 \\
\text { Temp: } 97.5-97.8\end{array}$ & $\begin{array}{l}\text { PR: } 85-95 \\
\text { dias: } 75-55 \\
\text { FBS: } 90\end{array}$ & \\
\hline 13 & Aug 12 & $\begin{array}{l}\text { SpO2: } 98 \\
\text { BP sys: } 137-141 \\
\text { Temp: } 97.4-97.9\end{array}$ & $\begin{array}{l}\text { PR: } 76-85 \\
\text { dias: } 75-90\end{array}$ & \\
\hline 14 & Aug 13 & $\begin{array}{l}\text { SpO2: } 98 \\
\text { BP sys: } 133-134 \\
\text { Temp: } 97.6-97.7 \\
\text { FBS: } 83\end{array}$ & $\begin{array}{l}\text { PR: } 85-90 \\
\text { dias: } 84 \\
\text { PPBS: } 135\end{array}$ & $\begin{array}{l}\text { Patient reported to have been } \\
\text { measuring blood glucose level } \\
\text { regularly - range FBS approx. } \\
110 \mathrm{mg} / \mathrm{dl} ; \text { PPBS approx. } \\
150-160 \mathrm{mg} / \mathrm{dl}) .\end{array}$ \\
\hline 15 & Aug 14 & $\begin{array}{l}\text { SpO2: } 99 \\
\text { BP: } 138 / 85\end{array}$ & $\begin{array}{l}\text { PR: } 85 \\
\text { Temp: } 98.2\end{array}$ & \\
\hline
\end{tabular}

Table 7: Progress of patient P04 and evolution of the treatment plan

\section{Detailed treatment plan and progress tracking for P04:}

The patient was asymptomatic at the time of first consultation.

History of the present illness was taken, which is as follows. She had no symptoms since the time of being tested positive for COVID on August 1 (sample shared on July 31). However, the patient had reported chronic constipation and (border-line) hypothyroidism. Considering the patient's age (75 years), and given the fact that she had been a patient of HTN since 5 years, DM Type II since 15 years and had been on insulin $(24 \mathrm{U})$ since the last $4-5$ years, she was considered to be in the high-risk group and given treatment as a preventive measure. The following allopthic supplements were prescribed: Vitamin C, Zinc, multivitamin.

The following allopathic medicines were being taken regularly by the patient: Janumet 50/500, Nebicard T, Glemi M2, Citanil, Ecosprin 75. The patient's FBS (recorded 3-4 days prior to the first consultation) was reported to be $125 \mathrm{mg} / \mathrm{dl}$ and HbA1c (as on June 2020) was reported to be approximately $8 \%$. Based on the patient's medical history, the following prescription for the Integrative Therapy was given as a preventive measure: 
Ayurvedic medicines prescribed: Sanshamani vati (1 BD), Laghumalini vasant (1 BD), Ayush Kwath (BD), Aloe vera + Amla juice.

975 Yoga Protocol: Sukshma Vyayama (upper and lower body parts); Breathing exercises (4 exercises with 5 iterations in each); asanas: (Triyaktadasana, Trikonasana, Ardhachakrasana, Parshva Sukhasana, Utthana Mandukasana,Ardhaushtrasana, Bhujangasana, Shalabhasana, Ardhahalasana with one leg folded, Pawanmuktasana); Shavasana; Pranayama (sectional breathing and full yogic breathing, Bhramari, Anulom-vilom, Udgeet); Dhyana.

The patient was almost fully compliant during the first half of the course of treatment, and remained asymptomatic. However, during the second half, the patient started having constipation and gastric issues (which had been a chronic problem with her); subsequently, she was advised to discontinue the medicines but continue with the yoga sessions. This not only helped her overcome the gastric trouble, but also helped her with joint movement and muscular stiffness. At the end of the treatment, it was reported that she could now sit in cross-legged position - something she was not able to do prior to the treatment. She remained asymptomatic throughout the treatment. The patient's faith in Ayurveda and Yoga was evident in the fact that at the end of the treatment Ayurvedic consultation for DM was sought, which was duly provided after observing the most recent $\mathrm{HbA1c}$ report available and fasting $+\mathrm{PP}$ reports on following three successive days.

Details of the patient's progress and compliance during the course of the treatment are given below in Table 8

\begin{tabular}{|c|l|ll|l|}
\hline Day & Date & Vital Measures & General Remarks \\
\hline \hline 0 & Aug 1 & SpO2: 93-95 & PR: 73-77 & Patient found to be asymptomatic \\
\hline 1 & Aug 2 & SpO2: 93-97 & PR: 74-79 & Patient remained asymptomatic \\
\hline 2 & Aug 3 & SpO2: 94-99 & PR: 63-79 & $\begin{array}{l}\text { Vitals chart shared by the patient; SpO2 ob- } \\
\text { served to be dropping below 95 on several occa- } \\
\text { sions. }\end{array}$
\end{tabular}




\begin{tabular}{|c|c|c|c|c|}
\hline 3 & Aug 4 & $\begin{array}{l}\text { SpO2: } 93-98 \\
\text { FBS: } 299\end{array}$ & PR: $67-80$ & $\begin{array}{l}\text { Prone position asanas makarasana and shithi- } \\
\text { lasana recommended for improving SpO2 level; } \\
\text { no other symptom observed. }\end{array}$ \\
\hline 4 & Aug 5 & SpO2: $91-97$ & PR: $68-86$ & Patient continues to be asymptomatic. \\
\hline 5 & Aug 6 & $\begin{array}{l}\text { SpO2: } 94-96 \\
\text { FBS: } 96\end{array}$ & PR: 66-73 & Patient continues to be asymptomatic. \\
\hline 6 & Aug 7 & SpO2: $95-98$ & PR: $66-76$ & $\begin{array}{l}\text { Patient vomited twice - diagnosed to be due to } \\
\text { acidity; SpO2 remained } 95 \text { or above this day } \\
\text { onwards. }\end{array}$ \\
\hline 7 & Aug 8 & $\begin{array}{l}\text { SpO2: } 95-99 \\
\text { BP: } 153 / 73\end{array}$ & PR: 64-96 & $\begin{array}{l}\text { Patient continues to be } \\
\text { asymptomatic. }\end{array}$ \\
\hline 8 & Aug 9 & $\begin{array}{l}\text { SpO2: } 96 \\
\text { BP: } 153 / 79\end{array}$ & PR: $68-72$ & $\begin{array}{l}\text { Patient complained of headache and constipa- } \\
\text { tion (possibly due to gastric upset) and skipped } \\
\text { the yoga session; discontinued Sanshamani vati } \\
\text { and Laghumalini vasant hereon; SpO2 level re- } \\
\text { mained } 96 \text { or above hereon. }\end{array}$ \\
\hline 9 & Aug 10 & $\begin{array}{l}\text { SpO2: } 97 \\
\text { BP: } 156 / 78\end{array}$ & $\begin{array}{l}\text { PR: } 70 \\
\text { FBS: } 96\end{array}$ & $\begin{array}{l}\text { Patient discontinued medicines; did only yoga; } \\
\text { increased intake of liquid diet - juice, coconut } \\
\text { water, tulsi water etc. for constipation. }\end{array}$ \\
\hline 10 & Aug 11 & SpO2: $96-97$ & PR: $62-67$ & Only yoga done \\
\hline 11 & Aug 12 & SpO2: 96 & PR: $63-64$ & \\
\hline 12 & Aug 13 & $\begin{array}{l}\text { SpO2: } 96-97 \\
\text { BP: } 162 / 77\end{array}$ & $\begin{array}{l}\text { PR: } 61-70 \\
\text { FBS: } 194\end{array}$ & $\begin{array}{l}\text { Patient sought Ayurvedic intervention for dia- } \\
\text { betes - duly done. }\end{array}$ \\
\hline 13 & Aug 14 & SpO2: 97 & PR: $62-67$ & \\
\hline 14 & Aug 15 & $\begin{array}{l}\text { SpO2: } 97 \\
\text { BP: } 152 / 74 \\
\text { FBS: } 75\end{array}$ & $\begin{array}{l}\text { PR: } 60 \\
\text { PPBS: } 311\end{array}$ & \\
\hline 15 & Aug 16 & $\begin{array}{l}\text { SpO2: } 98 \\
\text { BP: } 135 / 63 \\
\text { FBS: } 131\end{array}$ & PPBS: 234 & \\
\hline
\end{tabular}




\begin{tabular}{|c|c|c|c|c|}
\hline 16 & Aug 17 & $\begin{array}{l}\text { SpO2: } 96 \\
\text { BP: } 135 / 64 \\
\text { FBS: } 110\end{array}$ & $\begin{array}{l}\text { PR: } 60 \\
\text { PPBS: } 267\end{array}$ & \\
\hline 17 & Aug 18 & $\begin{array}{l}\text { SpO2: } 96 \\
\text { BP: } 148 / 81 \\
\text { FBS: } 105\end{array}$ & $\begin{array}{l}\text { PR: } 69 \\
\text { PPBS: } 209\end{array}$ & $\begin{array}{l}\text { The blood glucose levels observed from Aug } 15 \\
\text { to Aug } 18 \text { were used for prescribing Ayurvedic } \\
\text { medicines for DM (as requested by the patient) }\end{array}$ \\
\hline
\end{tabular}

Table 8: Progress of patient P04 and evolution of the treatment plan

\section{Detailed treatment plan and progress tracking for P05:}

The patient presented with the following symptoms at the time of the first consultation: sore throat, fever (mild) and weakness. He had developed the following symptoms: sore throat, mild fever and weakness on August 1. Following this, he underwent RAT on August 3 and was tested positive for COVID. Subsequently, he was prescribed the following allopathic medication - Doxy, Vitamin C (Citravite XT), Multivitamin (Vitneurin CZS), Cyra D, Zinc.

The following allopathic medicines were being regularly taken by the patient for Coronary artery disease (CAD), HTN, Tachycardia and acidity: tab. Dilzem60, tab. Ecosprin 75, Zinetac.

Based on the symptoms at the time of the first consultation and the patient's medical history, the patient was prescribed the following Ayurvedic medication on August 3: Sanshamani Vati (1 BD), Laghumalini Vasant (1 BD). The symptoms persisted till August 9, and ceased after that (except that the patient continued to experience weakness). However, he joined the Integrative Therapy on August 10, when the only symptom prevailing was weakness. During the first consultation, the patient reported his $\mathrm{SpO} 2$ level to be consistently around 97-98. The patient continued to take the Ayurvedic medicines mentioned above. The details of the Yoga protocol designed for the patient are given below.

Yoga Protocol: Sukshma Vyayama (upper and lower body parts); Breathing exercises (4 exercises with 5 iterations in each); asanas: (Tadasana, Triyaktadasana, Trikonasana, Ardhachakrasana, Parshva Sukhasana, Sukhasana twist, Utthana Mandukasana, Bhujangasana, Shalabhasana, Ardhahalasana, Pawan- 


\section{Detailed treatment plan and progress tracking for P06:}

The patient was tested positive for COVID on Aug 4 through RAT, and presented with the following symptoms at the time of the first consultation done on the same day: fever, cough with sputum, weakness. She also reported to be hav-

muktasana); Shavasana; Pranayama (sectional breathing and full yogic breathing, Bhramari, Anulom-vilom, Udgeet); Dhyana.

The patient was almost fully compliant with respect to Ayurvedic medicines as well as Yoga sessions. He regularly took medicines and attended all the yoga sessions. Moreover, he continued to practice yoga with the help of the recorded video provided to him. Within about 6 days of joining the Integrative Therapy, the patient reported complete relief and rejuvenation. He was extremely happy with the yoga sessions and shared his experiences with both the Ayurveda doctor and the Yoga therapist.

ing gastric upset, chronic constipation and tendency of anxiety. Subsequently, she was advised the following allopathic medication: Citravite XT, Cap. Doxy, Vitneurin CZS, Cyra D, Zinc, Azee.

The following allopathic medicines were being taken regularly by the patient for HTN: Azilzu 40 (given half a dose and on alternate days).

1040 Based on the symptoms at the time of the first consultation and the patient's medical history, the following Ayurvedic medicines were prescribed on Aug 4: Laghumalini vasant, Sanshamani vati, kadha. Later, as the illness progressed, Talishadi churna was added to the prescription.

The patient joined the Integrative Therapy on Aug 10. At the time of joining the IT, the patient reported to have fever $\left(<100^{\circ} \mathrm{F}\right)$, cough with sputum, weakness; the SpO2 level was reported to remain above 95 . She continued with ayurvedic medicines specified above. The details of the yoga protocol suggested for the patient are given below:

Yoga Protocol: Sukshma Vyayama (upper and lower body parts); Breathing exercises (4 exercises with 5 iterations in each); Asanas: (tadasana, triyak- 
tadasana, trikonasana, ardhachakrasana,parshva sukhasana, sukhasana twist, utthana mandukasana, bhujangasana, shalabhasana, ardhahalasana, pawanmuktasana); Shavasana; Pranayama (sectional breathing and full yogic breathing, bhramari, anulom-vilom, udgeet); Dhyana. The patient was almost fully compliant to the prescribed treatment. She regularly took the medicines and attended all the yoga sessions. Moreover, she continued to practice yoga with the help of the recorded video provided to her after the completion of the treatment.

Details of the patient's progress and compliance during the course of the treatment are given below in Table 9

\begin{tabular}{|c|c|c|c|}
\hline Day & Date & Vital Measures & General Remarks \\
\hline 0 & Aug 4 & & $\begin{array}{l}\text { Symptoms presented: fever, cough with } \\
\text { sputum, weakness }\end{array}$ \\
\hline 6 & Aug 10 & $\begin{array}{l}\text { SpO2: }>95 \\
\text { temp: }<100.0\end{array}$ & $\begin{array}{l}\text { Patient complained of fever, weakness, } \\
\text { cough with sputum (severe); besides, } \\
\text { gastric upset and acidity were also re- } \\
\text { ported }\end{array}$ \\
\hline 7 & Aug 11 & $\begin{array}{l}\text { SpO2: } 94-95 \quad \text { PR: } 77-80 \\
\text { temp: } 99.0-99.5\end{array}$ & \\
\hline 8 & Aug 12 & $\begin{array}{l}\text { SpO2: } 93-97 \quad \text { PR: } 79-88 \\
\text { temp: } 98.0-99.4 \\
\text { BP sys:120-137, dias: } 72-84\end{array}$ & $\begin{array}{l}\text { Patient had fever and bouts of } \\
\text { cough twice; complained of anxi- } \\
\text { ety; Makarsana and Shithilasana } \\
\text { suggested due to low SpO2 }\end{array}$ \\
\hline 9 & Aug 13 & $\begin{array}{l}\text { SpO2: } 96-97 \quad \text { PR: } 69-84 \\
\text { temp: } 98.0-98.4 \\
\text { BP sys:130-146, dias: } 77-86\end{array}$ & $\begin{array}{l}\text { Patient's condition reported to be much } \\
\text { better than the previous day; no } \\
\text { medicine taken for fever; cough bet- } \\
\text { ter than previous day; yoga nidra recom- } \\
\text { mended }\end{array}$ \\
\hline 10 & Aug 14 & $\begin{array}{l}\text { SpO2: } 96-97 \quad \text { PR: } 70-81 \\
\text { temp: } 98.3-98.4 \\
\text { BP sys:113-131, dias:70-76 }\end{array}$ & $\begin{array}{l}\text { Symptoms relieved to quite an extent; } \\
\text { oxygen level improved; reported to have } \\
\text { done yoga nidra and enjoying yoga; } \\
\text { slept well }\end{array}$ \\
\hline
\end{tabular}




\begin{tabular}{|c|c|c|c|}
\hline 11 & Aug 15 & $\begin{array}{l}\text { SpO2: } 95-98 \quad \text { PR: } 77-84 \\
\text { temp: } 98.0-98.5 \\
\text { BP sys:128-137, dias: } 62-83\end{array}$ & Same as before \\
\hline 12 & Aug 16 & $\begin{array}{l}\text { SpO2: } 96-97 \quad \text { PR: } 77-81 \\
\text { temp: } 98.2-98.5 \\
\text { BP: } 123 / 71\end{array}$ & $\begin{array}{l}\text { Mild cough; weakness - felt tired for a } \\
\text { while after climbing stairs; no more anx- } \\
\text { iety; overall feeling much better, sleep- } \\
\text { ing well }\end{array}$ \\
\hline 13 & Aug 17 & $\begin{array}{l}\text { SpO2: } 96-97 \quad \text { PR: } 67-80 \\
\text { temp: } 98.2-98.5 \\
\text { BP: } 134 / 81\end{array}$ & $\begin{array}{l}\text { started Talishadi churna and San- } \\
\text { shamani vati (SOS) }\end{array}$ \\
\hline 14 & Aug 18 & $\begin{array}{l}\text { SpO2: } 96-98 \quad \text { PR: } 77-86 \\
\text { temp: } 98.2-98.4\end{array}$ & $\begin{array}{l}\text { symptoms same as above; took Tal- } \\
\text { isadi churna only once due to discom- } \\
\text { fort caused by spicy }\end{array}$ \\
\hline 15 & Aug 19 & $\begin{array}{l}\text { SpO2: } 97-98 \quad \text { PR: } 79-84 \\
\text { temp: } 98.4-98.5\end{array}$ & $\begin{array}{l}\text { Patient advised to take Talisadi churna } \\
\text { twice with reduced quantity; sleeping } \\
\text { well; eating properly }\end{array}$ \\
\hline 16 & Aug 20 & $\begin{array}{l}\text { SpO2: } 96-98 \quad \text { PR: } 74-84 \\
\text { temp: } 98.0-98.4\end{array}$ & $\begin{array}{l}\text { Patient advised to do nasya with til } \\
\text { oil; no fever medicine being taken for } \\
\text { past few days; reported } 50 \% \text { relief in } \\
\text { weakness; } 80 \% \text { relief in cough; relief } \\
\text { in constipation without any additional } \\
\text { medicines }\end{array}$ \\
\hline 17 & Aug 21 & $\begin{array}{l}\text { SpO2: } 96-98 \quad \text { PR: } 74-87 \\
\text { temp: } 98.2-98.4\end{array}$ & \\
\hline 18 & Aug 22 & $\begin{array}{l}\text { SpO2: } 96-98 \quad \text { PR: } 71-81 \\
\text { temp: } 98.3-98.5\end{array}$ & \\
\hline 19 & Aug 23 & $\begin{array}{l}\text { SpO2: } 96-98 \quad \text { PR: } 71-80 \\
\text { temp: } 98.2-98.4\end{array}$ & \\
\hline 23 & Aug 27 & & $\begin{array}{l}\text { Nasya not followed; still taking tal- } \\
\text { ishadi churna and multivitamin; no } \\
\text { cough; mild weakness; overall good; re- } \\
\text { covered according to the patient. Still } \\
\text { doing yoga regularly; yoga nidra at } \\
\text { times }\end{array}$ \\
\hline
\end{tabular}


Table 9: Progress of patient P06 and evolution of the treatment plan

\section{Detailed treatment plan and progress tracking for P07:}

The patient presented with the following symptoms at the time of the first con-

below. Besides the above medicines, the patient had been taking kadha twice a day on his own

Yoga Protocol: Sukshma Vyayama (upper and lower body parts without neck rotation); Breathing exercises (4 exercises with 5 iterations in each); asanas:

dukasana, supported Supta Vajrasana, Vakrasana, Pawanmuktasana; Shavasana; Pranayama (sectional breathing and full yogic breathing, Bhramari, Anulomvilom, Udgeet); Dhyana.

The patient was largely compliant. He stopped taking the Ayurvedic medicines 
days later to resume the medication, when he started taking half the prescribed dosage. Another couple of days later he resumed the full dosage. Also, he missed some of the guided yoga sessions. By Sep 1, the patient reported complete relief and rejuvenation.

1095

Details of the patient's progress and compliance during the course of the treatment are given below in Table 10 . The patient underwent RTPCR on September 7, which was negative.

\begin{tabular}{|c|c|c|c|}
\hline Day & Date & Vital Measures & General Remarks \\
\hline 0 & Aug 15 & & $\begin{array}{l}\text { Patient had developed fever, body-ache and } \\
\text { cough on Aug. 11, was on allopathic medication. } \\
\text { Present symptoms: fever, sore throat, cough with } \\
\text { sputum. }\end{array}$ \\
\hline 1 & Aug 16 & & Patient not approachable; vitals not shared \\
\hline 2 & Aug 17 & & $\begin{array}{l}\text { Sudarshan ghanvati added to prescription; symp- } \\
\text { toms prevailed; patient appeared to have anxi- } \\
\text { ety over health issues; vitals not shared; not yet } \\
\text { ready to do yoga }\end{array}$ \\
\hline 3 & Aug 18 & $\begin{array}{l}\text { SpO2: } 97, \mathrm{PR}: 86 \\
\text { temp: } 100\end{array}$ & $\begin{array}{l}\text { Symptoms prevailed; new symptom-loose stools; } \\
\text { patient agreed to begin yoga sessions on Aug } 19\end{array}$ \\
\hline 4 & Aug 19 & $\begin{array}{l}\text { SpO2: } 98 \\
\text { PR: } 80 \\
\text { temp: } 97\end{array}$ & $\begin{array}{l}\text { Patient complained of mouth ulcers and upset } \\
\text { stomach (loose motions); Clotrin mouth paint, } \\
\text { Oro-T, Vitneurin CZS (1 OD) suggested. Patient } \\
\text { attributed stomach problem to the medicines and } \\
\text { discontinued most medicines; increased intake of } \\
\text { liquid (lassi, chhachh, lemon water); did not } \\
\text { show up for yoga session }\end{array}$ \\
\hline 5 & Aug 20 & $\begin{array}{l}\text { SpO2: } 97 \\
\text { PR: } 78 \\
\text { temp: } 97\end{array}$ & $\begin{array}{l}\text { Patient complained of cough, upset stomach, } \\
\text { headache, bad taste, reduced appetite and ex- } \\
\text { treme weakness; reported improvement in fever } \\
\text { and having eaten solid food after many days; } \\
\text { sleep cycle highly disturbed - yoga nidra was rec- } \\
\text { ommended for the same }\end{array}$ \\
\hline
\end{tabular}




\begin{tabular}{|c|c|c|c|}
\hline 6 & Aug 21 & $\begin{array}{l}\text { SpO2: } 95 \\
\text { PR: } 69\end{array}$ & $\begin{array}{l}\text { Patient reported to have had severe headache in } \\
\text { the morning - took medicine himself and slept } \\
\text { again; reported feeling of restlessness in general } \\
\text { and tiredness after yoga; all symptoms persist. } \\
\text { Counselled and advised to resume medicines with } \\
\text { half dosage, and have light meals with short gaps }\end{array}$ \\
\hline 7 & Aug 22 & $\begin{array}{l}\text { SpO2: } 97-99 \\
\text { PR: } 75-82 \\
\text { BP: } 118 / 84\end{array}$ & $\begin{array}{l}\text { Patient complained of headache and nausea in } \\
\text { the morning, unable to eat, bad taste and upset } \\
\text { stomach; syrup Himcocid ( } 2 \text { teaspoons thrice a } \\
\text { day) added to the prescription, Zoemit and Di- } \\
\text { arex advised (only } 1 \text { tablet each); skipped yoga }\end{array}$ \\
\hline 8 & Aug 23 & $\begin{array}{l}\text { SpO2: } 95 \\
\text { PR: } 79\end{array}$ & $\begin{array}{l}\text { Full dose of medicines resumed; reported } 30 \% \\
\text { improvement in symptoms, appetite improving; } \\
\text { skipped yoga }\end{array}$ \\
\hline 9 & Aug 24 & & Vitals not shared \\
\hline 10 & Aug 25 & $\begin{array}{l}\text { SpO2: } 95-96 \\
\text { PR: } 66-83\end{array}$ & $\begin{array}{l}\text { Patient reported improvement in symptoms with } \\
\text { appetite getting normal- he sounded much bet- } \\
\text { ter; stomach still a bit upset; little cough, weak- } \\
\text { ness, bad taste persist. Advised to do yoga more } \\
\text { regularly, practise yoga nidra and prone position } \\
\text { asanas }\end{array}$ \\
\hline 11 & Aug 26 & $\begin{array}{l}\text { SpO2: } 97-98 \\
\text { PR: } 72-82\end{array}$ & $\begin{array}{l}\text { Patient reported } 50 \% \text { improvement - particularly } \\
\text { cough improved; weakness persisted }\end{array}$ \\
\hline 12 & Aug 27 & & $\begin{array}{l}\text { No cough, stomach improved, but loss of appetite } \\
\text { persists and still feels weak; overall worse than } \\
\text { the previous day; vitals not shared }\end{array}$ \\
\hline 13 & Aug 28 & $\begin{array}{l}\text { SpO2: } 97 \\
\text { PR: } 94 \\
\text { BP: } 115 / 78\end{array}$ & $\begin{array}{l}\text { Mild cough again, stomach still little upset, } \\
\text { weakness better, appetite improved. Overall } 70 \% \\
\text { improvement }\end{array}$ \\
\hline 14 & Aug 29 & $\begin{array}{l}\text { SpO2: } 97-98 \\
\text { PR: } 94-100\end{array}$ & $\begin{array}{l}\text { Bitterness in mouth, throat persisted; stomach } \\
\text { still a little upset; cough quite improved; appetite } \\
\text { normal; liquid intake still increased }\end{array}$ \\
\hline 15 & Aug 30 & $\begin{array}{l}\text { SpO2: } 98 \\
\text { PR: } 83\end{array}$ & $\begin{array}{l}\text { Patient feels like } 80 \% \text { recovered; still having loose } \\
\text { motions (with normal frequency); mild bitterness } \\
\text { in taste but eating properly, mild weakness, no } \\
\text { cough }\end{array}$ \\
\hline
\end{tabular}




\begin{tabular}{|c|c|c|c|}
\hline 16 & Aug 31 & $\begin{array}{l}\text { SpO2: } 98-99 \\
\text { PR: } 76-85 \\
\text { BP sys: } 120-122 \\
\text { dias: } 78-83\end{array}$ & \\
\hline 17 & Sep 01 & $\begin{array}{l}\text { SpO2: } 98 \\
\text { PR: } 90\end{array}$ & $\begin{array}{l}\text { Medicines discontinued (dose completed); } 100 \% \\
\text { recovery reported }\end{array}$ \\
\hline 25 & Sep 09 & & RTPCR negative (sample given on Sep 07) \\
\hline
\end{tabular}

Table 10: Progress of patient P07 and evolution of the treatment plan

\section{Detailed treatment plan and progress tracking for P08:}

1100

The patient presented with the following symptoms at the time of the first consultation: fever, body ache. He was subsequently prescribed the following Ayurvedic medicines: Sudarshan Ghanvati (1 TDS), Immunity capsules (1 TDS), tab. Fifatrol (1 BD). The next day he complained of nausea and loss of taste (or bad taste as described by the patient), when he was prescribed Zoemit tab. He underwent RT-PCR the same day on August 24, and was found COVID positive.

The following medicine was being regularly taken by the patient for HTN: Amcard-AT.

Based on the patient's medical history and the symptoms presented, the following prescription for the Integrative Therapy was given:

Ayurvedic medicines and procedures prescribed: Laghumalini Vasant (1 BD), Sanshamani Vati (1 BD), Amynity (2 teaspoon BD), Chyavanprash, steam inhalation and gargles.

Yoga Protocol: Breathing exercises (2 exercises with 5 iterations in each); Sukshma Vyayama (upper and lower body parts); asanas with 2 rounds each: Parshva Sukhasana, Utthana Mandukasana, Ardha Halasana, Pawanmuktasana, Shavasana, Pranayama (sectional breathing and full yogic breathing, Bhramari, 
Anulom-vilom, Udgeet); Dhyana.

${ }_{1120}$ The patient was almost fully compliant. He kept taking half the prescribed dosage of medicines by mistake for the first few days, until this came to notice and he was accordingly suggested. He attended all the guided Yoga sessions and continued practising himself with the help of the recorded video provided to him. In a follow-up call after the treatment was over, he reported immense 1125 improvement in digestion (bowel movement) and stiffness in joints and muscles - the patient attributed this to Usha paan and Yoga. He was subsequently connected to a Yoga organization so that he could further pursue his yoga practice and continue to reap the benefits he had started experiencing.

Details of the patient's progress and compliance during the course of the treatment are given below in Table11. The patient underwent RTPCR on September 9 , which was negative.

\begin{tabular}{|c|c|c|c|}
\hline Day & Date & Vital Measures & General Remarks \\
\hline 0 & Aug 23 & & $\begin{array}{l}\text { Symptoms reported: fever, } \\
\text { body-ache }\end{array}$ \\
\hline 1 & Aug 24 & & $\begin{array}{l}\text { Symptoms reported: body- } \\
\text { ache, nausea, loss of taste } \\
\text { (later reported by the patient } \\
\text { as foul taste) }\end{array}$ \\
\hline 2 & Aug 25 & & $\begin{array}{l}\text { Symptoms reported: No } \\
\text { fever, loss of taste (later, re- } \\
\text { ported as bad taste), morning } \\
\text { nausea (feeling of vomiting } \\
\text { early in the morning) }\end{array}$ \\
\hline 3 & Aug 26 & $\begin{array}{l}\text { PR: } 66-72 \text { temp: } 98.5-99.4 \\
\text { BP sys: } 116-118 \text {, dias: } 82-89\end{array}$ & $\begin{array}{l}\text { Patient complained of morn- } \\
\text { ing nausea; SpO2 not re- } \\
\text { ported }\end{array}$ \\
\hline 4 & Aug 27 & $\begin{array}{l}\text { PR: } 72 \text { temp: } 99.4 \\
\text { BP } 126 / 88\end{array}$ & $\begin{array}{l}\text { Patient complained of morn- } \\
\text { ing nausea, mild heaviness } \\
\text { in throat; about } 75 \% \text { relief } \\
\text { reported; yoga nidra recom- } \\
\text { mended }\end{array}$ \\
\hline
\end{tabular}




\begin{tabular}{|c|c|c|c|}
\hline 5 & Aug 28 & $\begin{array}{l}\text { PR: } 71-76 \text { temp: } 98.6-99.3 \\
\text { BP sys: } 118-125 \text {, dias: } 80-86\end{array}$ & $\begin{array}{l}\text { Morning nausea and mild } \\
\text { heaviness in throat persist; } \\
\text { usha paan recommended }\end{array}$ \\
\hline 6 & Aug 29 & $\begin{array}{l}\text { SpO2: } 95-98 \quad \text { PR: } 70-78 \\
\text { temp: } 98.5-99.6 \\
\text { BP sys: } 125-129 \text {, dias: } 77-86\end{array}$ & $\begin{array}{l}\text { Improvement in morning nau- } \\
\text { sea; no more heaviness in } \\
\text { throat; started usha paan; re- } \\
\text { ported almost complete relief } \\
\text { with normal diet, appetite }\end{array}$ \\
\hline 7 & Aug 30 & $\begin{array}{l}\text { SpO2: } 96-98 \quad \text { PR: } 71-74 \\
\text { temp: } 97.9-98.9 \\
\text { BP sys: } 122-129 \text {, dias: } 84-87\end{array}$ & $\begin{array}{l}\text { Morning nausea not felt after } \\
\text { gargles; } 100 \% \text { relief and reju- } \\
\text { venated }\end{array}$ \\
\hline 8 & Aug 31 & $\begin{array}{l}\text { SpO2: } 95-98 \quad \text { PR: } 69-75 \\
\text { temp: } 97.9-99.2 \\
\text { BP sys: } 117-133 \text {, dias: } 83-87\end{array}$ & $\begin{array}{l}\text { Patient continues to be fine } \\
\text { with no symptoms. }\end{array}$ \\
\hline 9 & Sep 01 & $\begin{array}{l}\text { SpO2: } 96-98 \quad \text { PR: } 73-86 \\
\text { temp: } 98.2-100.6 \\
\text { BP sys: } 122-129 \text {, dias: } 80-86\end{array}$ & $\begin{array}{l}\text { Patient continues to feel fine } \\
\text { with no symptoms. }\end{array}$ \\
\hline 10 & Sep 02 & $\begin{array}{l}\text { SpO2: } 96-98 \quad \text { PR: } 77-85 \\
\text { temp: } 98.9-99 \\
\text { BP sys: } 120-128 \text {, dias: } 77-88\end{array}$ & $\begin{array}{l}\text { It was realised that the pa- } \\
\text { tient had been taking half } \\
\text { the prescribed dosage of both } \\
\text { Sanshamani Vati and Laghu- } \\
\text { malini Vasant ( } 1 \text { OD instead } \\
\text { of } 1 \text { BD); advised to start tak- } \\
\text { ing the full dose. }\end{array}$ \\
\hline 12 & Sep 04 & $\begin{array}{l}\text { SpO2: } 96-98 \quad \text { PR: } 77-79 \\
\text { temp: } 98.2-98.7 \\
\text { BP sys: } 116-123 \text {, dias: } 77-79\end{array}$ & $\begin{array}{l}\text { Patient started taking the full } \\
\text { dose of medicines as sug- } \\
\text { gested; continuing with steam } \\
\text { inhalation, gargles and usha } \\
\text { paan. No more feeling of } \\
\text { morning nausea. }\end{array}$ \\
\hline 13 & Sep 05 & $\begin{array}{l}\text { SpO2: } 96-98 \quad \text { PR: } 78-85 \\
\text { temp: } 98.4-99.3 \\
\text { BP sys: } 115-127 \text {, dias: } 74-86\end{array}$ & $\begin{array}{l}\text { Patient doing perfectly fine. } \\
\text { Practising yoga twice a day } \\
\text { since Sep } 04 \text { with the recorded } \\
\text { video provided. }\end{array}$ \\
\hline 14 & Sep 06 & $\begin{array}{l}\text { SpO2: } 96-98 \quad \text { PR: } 80-88 \\
\text { temp: } 98.6-99.2 \\
\text { BP sys: } 120-125 \text {, dias: } 80 \\
70\end{array}$ & $\begin{array}{l}\text { Patient doing perfectly fine. } \\
\text { Practising yoga twice a day } \\
\text { since Sep } 04 \text { with the recorded } \\
\text { video provided. }\end{array}$ \\
\hline
\end{tabular}




\begin{tabular}{|c|c|l|l|} 
& & & \\
\hline \multirow{2}{*}{16} & Sep 08 & $\begin{array}{l}\text { SpO2: 96-98 PR: 77-81 } \\
\text { temp: 97.5-98.8 } \\
\text { BP sys: 119-125, dias: 73-83 }\end{array}$ & $\begin{array}{l}\text { Patient happily doing yoga } \\
\text { twice everyday. Reported re- } \\
\text { markable improvement in di- } \\
\text { gestion. }\end{array}$ \\
\hline \multirow{2}{*}{18} & Sep 10 & $\begin{array}{l}\text { RTPCR negative (sample } \\
\text { given on September 9). }\end{array}$ \\
\hline
\end{tabular}

Table 11: Progress of patient P08 and evolution of the treatment plan

\section{Detailed treatment plan and progress tracking for P09:}

The patient presented with the following symptoms at the time of the first consultation: fever, mild cough and cold.

History of the present illness was taken, which is as follows. He had developed fever and cough on August 23, following which he consulted an Allopathic doctor and was prescribed the following medication - Azithromycin and PCM for 405 days. Subsequently he underwent RAT on Aug 26 and was tested positive. The patient was regularly taking the following medicines for his comorbidities: Rosuvastatin $10 \mathrm{mg}$ (for cholestrol); Meslazin 1BD (for Ulcerative Colitis); does a course of steroids (omnacotin) every year (starting $40 \mathrm{mg}$ for 7 days, then $35 \mathrm{mg}$ and so on upto $5 \mathrm{mg}$.; reported have used enema this year instead of steroids.

Based on the patient's medical history and the symptoms presented, the following prescription for the Integrative Therapy was given:

Ayurvedic medicines and procedures prescribed: Sudarshan Ghanvati (1 TDS for 5 days), Sanshamani vati (1 BD), Laghumalini vasant (1 BD), Chyavanprash (organic), Ayush Kwath, steam inhalation and gargles - all for 15 days; some addition was done to the prescription to address a new symptom later presented. The details have been furnished in Table 12 below.

Yoga Protocol: Sukshma Vyayama (upper and lower body parts); Breathing 
exercises ( 4 exercises with 5 iterations in each); asanas: Tadasana, TriyakTadasana, Trikonasana, Ardhachakrasana, Parshva Sukhasana, Utthana Mandukasana, Ardha Ustrasana, Vakrasana, Bhujangasana, Shalabhasana, Ardhahalasana, Pawanmuktasana, Shavasana; Pranayama (sectional breathing and full yogic breathing, Bhramari, Anulom-vilom, Udgeet); Dhyana.

The patient was almost fully compliant. He was regular in taking the medicines as well as attending the yoga sessions. Besides, the patient reported to be a spiritual seeker and a follower of a well-known tradition of yoga.

Details of the patient's progress and compliance during the course of the treatment are given below in Table 12 .

\begin{tabular}{|c|c|c|c|}
\hline Day & Date & Vital Measures & General Remarks \\
\hline 0 & Aug 26 & Temp: 97.7 & $\begin{array}{l}\text { Symptoms reported: fever, mild cold, cough } \\
\text { since past } 3 \text { days; no fever currently }\end{array}$ \\
\hline 1 & Aug 27 & $\begin{array}{l}\text { SpO2: } 97-98 \\
\text { temp: } 98.1-98.3\end{array}$ & $\begin{array}{l}\text { Patient did not develop fever, but felt fever- } \\
\text { ish and weak; cough and cold persist }\end{array}$ \\
\hline 2 & Aug 28 & $\begin{array}{l}\text { SpO2: } 98 \\
\text { PR: } 99-110 \\
\text { temp: } 98 .-98.2\end{array}$ & $\begin{array}{l}\text { Feverish feeling and weakness persist, cough } \\
\text { and cold better ( } 70 \% \text { improvement), appetite } \\
\text { reduced; felt better after taking PCM }\end{array}$ \\
\hline 3 & Aug 29 & $\begin{array}{l}\text { SpO2: } 97-98 \\
\text { PR: } 96 \\
\text { temp: } 98.1\end{array}$ & Weakness and reduced appetite reported \\
\hline 4 & Aug 30 & $\begin{array}{l}\text { SpO2: } 97-98 \\
\text { PR: } 99 \\
\text { temp: } 98.3-99.4\end{array}$ & $\begin{array}{l}\text { Mild fever recorded, mild cough, occasional } \\
\text { nasal blockage (usual problem), extreme } \\
\text { weakness, body-ache, appetite slightly re- } \\
\text { duced, bitterness in taste; also reported } \\
\text { morning sickness; overall condition worse } \\
\text { than the previous day; advised to do gargles } \\
\text { and steam inhalation twice a day }\end{array}$ \\
\hline 5 & Aug 31 & $\begin{array}{l}\text { SpO2: } 99 \quad \text { PR: } 90 \\
\text { temp: } 97.9\end{array}$ & $\begin{array}{l}\text { Occasional mild nasal and chest blockage; } \\
\text { weakness, body-ache, reduced appetite, bad } \\
\text { taste, morning sickness- symptoms persist }\end{array}$ \\
\hline 6 & Sep 1 & $\begin{array}{l}\text { SpO2: } 97-98 \\
\text { PR: } 82-87 \\
\text { temp: } 97.4-98.3\end{array}$ & $\begin{array}{l}\text { All symptoms persist; overall condition same } \\
\text { as before } \\
72\end{array}$ \\
\hline
\end{tabular}




\begin{tabular}{|c|c|c|c|}
\hline 7 & Sep 2 & $\begin{array}{l}\text { SpO2: } 98 \\
\text { PR: } 83-92 \\
\text { temp: } 97.3-98.4\end{array}$ & $\begin{array}{l}\text { All symptoms persist but improved; } 50 \% \text { - } \\
60 \% \text { improvement in overall condition }\end{array}$ \\
\hline 8 & Sep 3 & $\begin{array}{l}\text { SpO2: } 97-98 \\
\text { PR: } 82-88 \\
\text { temp: } 97.3-97.9\end{array}$ & $\begin{array}{l}\text { Mild cough, mild bitter taste, mild pain in } \\
\text { throat; weakness and reduced appetite per- } \\
\text { sist; overall condition improved }\end{array}$ \\
\hline 9 & Sep 4 & $\begin{array}{l}\text { SpO2: } 98 \\
\text { PR: } 82-86 \\
\text { temp: } 97.3-97.8\end{array}$ & Condition same as before \\
\hline 10 & Sep 5 & $\begin{array}{l}\text { SpO2: } 98 \\
\text { PR: } 92 \\
\text { temp: } 97.7\end{array}$ & $\begin{array}{l}\text { Mild cough, weakness, bad taste and morn- } \\
\text { ing sickness persist, appetite still not nor- } \\
\text { mal; throat pain improved by } 60 \%-70 \% \text {; re- } \\
\text { ported constipation since } 2-3 \text { days; Talishadi } \\
\text { churna and papaya suggested to the patient }\end{array}$ \\
\hline 11 & Sep 6 & $\begin{array}{l}\text { SpO2: } 98 \\
\text { PR: } 82-92 \\
\text { temp: } 96.8-97.9\end{array}$ & condition almost same as before \\
\hline 12 & Sep 7 & $\begin{array}{l}\text { SpO2: } 98 \\
\text { PR: } 88-90 \\
\text { temp: } 97.1-98.0\end{array}$ & $\begin{array}{l}\text { Very mild cough and morning sickness, relief } \\
\text { from constipation with papaya; other symp- } \\
\text { toms same as before; started taking Talishdi } \\
\text { churna for cough; overall } 70 \% \text { recovery re- } \\
\text { ported }\end{array}$ \\
\hline 13 & Sep 8 & $\begin{array}{l}\text { SpO2: } 99 \quad \text { PR: } 102 \\
\text { temp: } 97.8\end{array}$ & $\begin{array}{l}\text { all symptoms relieved except very mild cough } \\
\text { and weakness }\end{array}$ \\
\hline 14 & Sep 9 & $\begin{array}{l}\text { SpO2: } 98 \quad \text { PR: } 98 \\
\text { temp: } 98.2\end{array}$ & same as before \\
\hline 16 & Sep 11 & $\begin{array}{l}\text { SpO2: } 99 \text { PR: } 102 \\
\text { temp: } 97.9\end{array}$ & same as before \\
\hline $\begin{array}{l}17 \\
\text { to } \\
21\end{array}$ & $\begin{array}{l}\text { Sep } 12 \\
\text { to } \\
\text { Sep } 16\end{array}$ & & $\begin{array}{l}\text { Follow-up done till Sep } 16 \text { - very mild } \\
\text { cough and weakness persisted; patient felt fit } \\
\text { enough to join back work on Sep } 14 \text {; vitals re- } \\
\text { mained normal; reported constipation once } \\
\text { during this period; discontinued Talishadi } \\
\text { churna and started taking kali mirch+neem } \\
\text { powder+haldi powder+honey for dry cough; } \\
\text { reported } 90 \% \text { recovery on Sep } 16 \\
73\end{array}$ \\
\hline
\end{tabular}




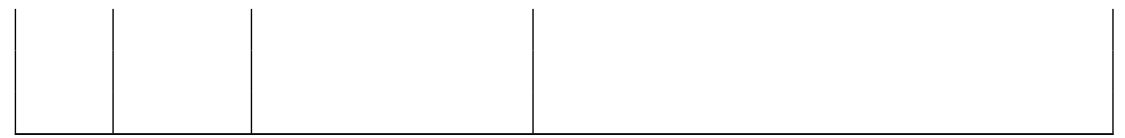

Table 12: Progress of patient P09 and evolution of the treatment plan

\section{Detailed treatment plan and progress tracking for P10:}

The patient presented with the following symptoms at the time of the first consultation: fever, sore throat, throat pain, cough with expectoration.

History of the present illness was taken, which is as follows. She had developed fever 2-3 days back, following which she underwent RT-PCR on August 26 and was tested positive. Subsequently, she was prescribed the following Allopathic medication: Azee 500 and Metrogyl 400 for 3 days, and Dolo (SOS).

The patient had been taking the following medicine regularly for HTN: Inzit 8 mg.

Based on the patient's medical history and the symptoms presented, the following prescription for the Integrative Therapy was given:

Ayurvedic medicines and procedures prescribed: Sudarshan Ghanvati (1 TDS) 5 days, Sanshamani vati (1 BD), Laghumalini vasant (1 BD), Chyavanprash (organic), Ayush Kwath, steam inhalation and gargles; some addition was done to the prescription to address a new symptom later presented. The details have been furnished in Table 13 below.

Yoga Protocol: Sukshma Vyayama (upper and lower body parts); Breathing exercises (4 exercises with 5 iterations in each); asanas: Tadasana, TriyakTadasana, Trikonasana, Ardhachakrasana, Parshva Sukhasana, Utthana Mandukasana, Ardha Ustrasana, Vakrasana, Bhujangasana, Shalabhasana, Ardhahalasana, Pawanmuktasana, Shavasana; Pranayama (sectional breathing and full yogic breathing, Bhramari, Anulom-vilom, Udgeet); Dhyana.

The patient was almost fully compliant. She was regular in taking the medicines as well as attending the yoga sessions. 
Details of the patient's progress and compliance during the course of the treatment are given below in Table 13 .

\begin{tabular}{|c|c|c|c|}
\hline Day & Date & Vital Measures & General Remarks \\
\hline 0 & Aug 27 & $\begin{array}{l}\text { SpO2: } 99 \\
\text { Temp: } 98.0-99.0\end{array}$ & $\begin{array}{l}\text { Initial symptoms: fever, sore throat, throat } \\
\text { pain, cough with sputum' loose motions re- } \\
\text { ported later during the day }\end{array}$ \\
\hline 1 & Aug 28 & $\begin{array}{l}\text { SpO2: } 99 \quad \text { PR: } 87 \\
\text { temp: } 98.2-99.0\end{array}$ & $\begin{array}{l}\text { Symptoms reported: fever (mild), sore } \\
\text { throat, throat pain, cough with sputum (feel- } \\
\text { ing like cough is stuck), body-ache, weakness, } \\
\text { loose motions, restlessness }\end{array}$ \\
\hline 2 & Aug 29 & $\begin{array}{l}\text { SpO2: } 98-99 \\
\text { PR: } 87-91 \\
\text { temp: } 98.4-99.0\end{array}$ & $\begin{array}{l}\text { Fever (mild), throat pain, cough and stomach } \\
\text { improved; patient reported to be feeling very } \\
\text { weak and restless; also developed headache }\end{array}$ \\
\hline 3 & Aug 30 & $\begin{array}{l}\text { SpO2: } 99 \quad \text { PR: } 85 \\
\text { temp: } 97.7\end{array}$ & $\begin{array}{l}\text { Throat pain reduced, occasional nasal block- } \\
\text { age (removed after steam inhalation), pain } \\
\text { in legs, weakness; other symptoms relieved; } \\
\text { overall } 70 \%-80 \% \text { relief; patient recalled that } \\
\text { the previous day she had forgotten to take } \\
\text { the regular BP medicine and attributed the } \\
\text { headache to this - did not experience ever af- } \\
\text { ter; suggested to note BP daily, but could } \\
\text { not arrange for the device }\end{array}$ \\
\hline 4 & Aug 31 & $\begin{array}{l}\text { SpO2: } 98 \\
\text { PR: } 90 \\
\text { temp: } 98.7\end{array}$ & $\begin{array}{l}\text { Throat pain persists, mild pain in legs and } \\
\text { mild weakness, occasional nasal/chest con- } \\
\text { gestion - relieved with steam inhalation; } \\
\text { overall better than the previous day }\end{array}$ \\
\hline 5 & Sep 1 & $\begin{array}{l}\text { SpO2: } 95-99 \\
\text { PR: } 72-87 \\
\text { temp: } 97.7-98.4\end{array}$ & $\begin{array}{l}\text { Mild throat pain; other symptoms same as } \\
\text { before }\end{array}$ \\
\hline 6 & Sep 2 & $\begin{array}{l}\text { SpO2: } 99 \\
\text { PR: } 77-89 \\
\text { temp: } 98.2-98.5\end{array}$ & $\begin{array}{l}\text { Throat pain much improved; mild pain in } \\
\text { legs and weakness; nasal/chest congestion } \\
\text { persists; overall } 80 \% \text { improvement reported }\end{array}$ \\
\hline 7 & Sep 3 & $\begin{array}{l}\text { SpO2: } 98-99 \\
\text { PR: } 70-79 \\
\text { temp: } 97.9-98.6\end{array}$ & $\begin{array}{l}\text { Feeling much better than the previous day; } \\
\text { mild throat pain and mild weakness persist }\end{array}$ \\
\hline 8 & Sep 4 & $\begin{array}{l}\text { SpO2: } 99 \\
\text { PR: } 76-86 \\
\text { temp: } 97.6-98.4\end{array}$ & $\begin{array}{l}90 \% \text { relief reported } \\
75\end{array}$ \\
\hline
\end{tabular}




\begin{tabular}{|c|c|c|c|}
\hline 9 & Sep 5 & $\begin{array}{l}\text { SpO2: } 96-97 \\
\text { PR: } 78-83 \\
\text { temp: } 97.7-98.2\end{array}$ & $\begin{array}{l}\text { Throat pain worsened because of speaking } \\
\text { for long in a meeting; also complained of } \\
\text { running nose and sneezing; LCZ suggested } \\
\text { by doctor (patient did not take), Talishadi } \\
\text { churna suggested for throat }\end{array}$ \\
\hline 10 & Sep 6 & $\begin{array}{l}\text { SpO2: } 99 \\
\text { PR: } 70-77 \\
\text { temp: } 97.7-98.1\end{array}$ & \\
\hline 11 & Sep 7 & $\begin{array}{l}\text { SpO2: } 98 \\
\text { PR: } 85 \\
\text { temp: } 98.2\end{array}$ & $\begin{array}{l}\text { Complete relief reported; no symptoms per- } \\
\text { sist }\end{array}$ \\
\hline 12 & Sep 8 & $\begin{array}{l}\text { SpO2: } 98 \\
\text { PR: } 96 \\
\text { temp: } 98.2\end{array}$ & $\begin{array}{l}\text { Talishadi churna started; patient continues } \\
\text { to be in good condition }\end{array}$ \\
\hline 15 & Sep 11 & $\begin{array}{l}\text { SpO2: } 99 \quad \text { PR: } 76 \\
\text { temp: } 97.8\end{array}$ & \\
\hline
\end{tabular}

Table 13: Progress of patient P10 and evolution of the treatment plan

\section{Detailed treatment plan and progress tracking for P11:} sultation: diarrhea (loose stools with increased frequency).

History of the present illness was taken, which is as follows. He had developed fever a few days back, following which he underwent RT-PCR on August 26 and was tested positive. Subsequently, he was prescribed the following Allopathic medication: Immuxen (Vitamin C, Zinc - 1 OD), VSL \#3 (probiotic $1 \mathrm{BD})$. Besides, the patient also reported to be taking the following Ayurvedic medicines at the time of the first consultation: Coronil tab. (2 BD), Swasari vati $(2 \mathrm{BD})$

The patient had been taking the following medicine regularly for his comor- 
Details of the patient's progress and compliance during the course of the treatment are given below in Table14. The patient underwent RTPCR on September 16, which was negative.

\begin{tabular}{|c|c|l|l|}
\hline Day & Date & Vital Measures & General Remarks \\
\hline \hline 0 & Sep 01 & $\begin{array}{l}\text { SpO2: } 97 \quad \text { PR: } 75 \\
\text { temp: } 96.8\end{array}$ & Initial symptoms: diarrhea \\
\hline 1 & Sep 02 & $\begin{array}{l}\text { SpO2: } 98 \quad \text { PR: } 72-87 \\
\text { temp: } 96.1-96.9\end{array}$ & $\begin{array}{l}\text { Frequency normal but stools still } \\
\text { loose; no other symptoms reported. }\end{array}$ \\
\hline 2 & Sep 03 & $\begin{array}{l}\text { SpO2: } 97-98 \\
\text { PR: } 71-74 \quad \text { BP: } 135 / 80 \\
\text { temp: } 95.6-96.3\end{array}$ & Same as the previous day.
\end{tabular}




\begin{tabular}{|c|c|c|c|}
\hline 3 & Sep 04 & $\begin{array}{l}\text { SpO2: } 97-98 \\
\text { PR: } 66-77 \quad \text { BP: } 138 / 82 \\
\text { temp: } 95.6-96.7\end{array}$ & $\begin{array}{l}\text { Diarrhea persists - frequency again in- } \\
\text { creased; patient also reported mild } \\
\text { stomach ache. However, he was ob- } \\
\text { served to be consuming heavy and } \\
\text { difficult-to-digest food items and was } \\
\text { advised against it. Received discharge } \\
\text { from govt. home isolation. }\end{array}$ \\
\hline 4 & Sep 05 & $\begin{array}{l}\text { SpO2: } 97-98 \\
\text { PR: } 64-88 \quad \text { BP: } 138 / 80 \\
\text { temp: } 95.1-97.7\end{array}$ & $\begin{array}{l}\text { Patient reported to have started } \\
\text { course of an Allopathic medicine, Zen- } \\
\text { flox oz, since the previous night. }\end{array}$ \\
\hline 5 & Sep 06 & $\begin{array}{l}\text { SpO2: } 97-98 \quad \text { PR: } 65-77 \\
\text { temp: } 97.3-97.5\end{array}$ & \\
\hline 6 & Sep 07 & $\begin{array}{l}\text { SpO2: } 97-98 \\
\text { PR: } 70-75 \\
\text { temp: } 97.3-97.5\end{array}$ & $\begin{array}{l}\text { In the morning, patient reported re- } \\
\text { lief with almost normal stools; he re- } \\
\text { ported to be eating light food such } \\
\text { as salted daliya khichdi, coconut wa- } \\
\text { ter, fruits etc. However, later at night } \\
\text { he reported trouble again with semi- } \\
\text { formed stools thrice since evening. He } \\
\text { was advised to start taking the pre- } \\
\text { scribed Ayurvedic medicine thrice (in- } \\
\text { stead of twice a day); however, the } \\
\text { patient discontinued all medicines ex- } \\
\text { cept the multivitamin and the one he } \\
\text { had been taking regularly for HTN. }\end{array}$ \\
\hline 7 & Sep 8 & $\begin{array}{l}\text { SpO2: } 97 \\
\text { PR: } 77-85 \\
\text { temp: } 97.3-98.3\end{array}$ & $\begin{array}{l}\text { Stomach still upset - had loose stools } \\
\text { twice; also reported mild stomach } \\
\text { ache. Again suggested to start tak- } \\
\text { ing the prescribed medicine thrice, } \\
\text { have light meals, and control anxiety } \\
\text { through regular practice of Yoga and } \\
\text { Pranayama. Patient reported to be } \\
\text { having papaya - advised not to. }\end{array}$ \\
\hline 8 & Sep 9 & $\begin{array}{l}\text { SpO2: } 97 \quad \text { PR: } 66-70 \\
\text { temp: } 97.5-97.8\end{array}$ & $\begin{array}{l}\text { Condition same as the previous day; } \\
\text { started taking medicine prescribed } \\
\text { under Integrative Therapy thrice (as } \\
\text { suggested); all other medicines still } \\
\text { discontinued. }\end{array}$ \\
\hline
\end{tabular}




\begin{tabular}{|c|c|c|c|}
\hline 9 & Sep 10 & $\begin{array}{l}\text { SpO2: } 98 \quad \text { PR: } 78 \\
\text { temp: } 97.4\end{array}$ & $\begin{array}{l}\text { Condition same as before; RTPCR } \\
\text { still positive; Yoga Nidra recom- } \\
\text { mended }\end{array}$ \\
\hline 10 & Sep 11 & $\begin{array}{l}\text { SpO2: } 97 \quad \text { PR: } 74 \\
\text { temp: } 97.5\end{array}$ & $\begin{array}{l}\text { Giloy started on doctor's recommen- } \\
\text { dation; Zinc and Vitamin C also } \\
\text { started }\end{array}$ \\
\hline 11 & Sep 12 & $\begin{array}{l}\text { SpO2: } 97-98 \quad \text { PR: } 71-73 \\
\text { temp: } 96.7-97.4\end{array}$ & $\begin{array}{l}\text { Reported improvement in diarrhea } \\
\text { (semi-formed stool only once); also re- } \\
\text { ported better sleep after Yoga Nidra }\end{array}$ \\
\hline 12 & Sep 13 & & Same as the previous day. \\
\hline 13 & Sep 14 & & $\begin{array}{l}\text { Condition worsened again - reported } \\
\text { to have had motion thrice with mild } \\
\text { pain in stomach. }\end{array}$ \\
\hline 15 & Sep 16 & & $\begin{array}{l}\text { RTPCR negative but stomach } \\
\text { still upset; prescription revised } \\
\text { by the doctor - Kutajarishta ( } 3 \\
\text { tsp)+Jirakadyarishta ( } 3 \text { tsp) mixed } \\
\text { with } 6 \text { tsp lukewarm water to be } \\
\text { taken BD after meals. }\end{array}$ \\
\hline
\end{tabular}

Table 14: Progress of patient P11 and evolution of the treatment plan

\section{Detailed treatment plan and progress tracking for P12:}

The patient presented with the following symptoms at the time of the first consultation, which was done at an Ayurveda hospital: fever and body-ache. After developing symptoms on Sep 01, she underwent RAT on Sep 02, was found positive and got admitted to the Ayurveda hospital the same day. Subsequently, the patient was advised the following Ayurvedic medicines: Nagaradi Kwath $40 \mathrm{ml}$ BD, Amalaki churna 3gm BD, Sanshamani gutika 2 BD, Kantakari Avaleha 2 tsp TDS for cough; some medicines were added to the prescription during the course of treatment for new symptoms presented by the patient. The details have been furnished in Table 15. Besides, the patient was advised to take PCM 1240 (SOS). 
The patient had been taking the following medicine regularly for her comorbidities: Ecosprin.

The patient joined the Integrative Therapy on Sep 4, when she presented the following symptoms: fever, body-ache and headache. While she continued to take the above Ayurvedic medicines, based on the patient's medical history and the symptoms presented, the following Yoga protocol was prescribed for her: Yoga Protocol: Breathing exercises (4 exercises with 5 iterations in each); Sukshma Vyayama (upper and lower body parts); asanas: Parshva Sukhasana, Sukhasana twist, Utthana Mandukasana, Shavasana; Pranayama (sectional breathing and full yogic breathing, Bhramari, Anulom-vilom, Udgeet); Dhyana.

The patient was almost fully compliant. She was regular in taking the medicines as well as attending the yoga sessions. In fact, she is a regular yoga practitioner. ment are given below in Table 15 .

\begin{tabular}{|c|c|c|c|}
\hline Day & Date & Vital Measures & General Remarks \\
\hline 0 & Sep 02 & & Symptoms reported: fever, body-ache \\
\hline 2 & Sep 04 & & $\begin{array}{l}\text { Symptoms reported: fever, body- } \\
\text { ache, headache; vitals not shared }\end{array}$ \\
\hline 3 & Sep 05 & & same as the previous day \\
\hline 4 & Sep 06 & $\begin{array}{l}\text { SpO2: } 97-99 \quad \text { PR: } 97-112 \\
\text { BP: } 120 / 81 \\
\text { temp: } 99.3-99.8\end{array}$ & all symptoms reported persist \\
\hline 5 & Sep 07 & $\begin{array}{l}\text { SpO2: } 97-98 \quad \text { PR: } 78-112 \\
\text { temp: } 98.6-100\end{array}$ & $\begin{array}{l}\text { Fever persists; no body-ache or } \\
\text { headache; mild weakness reported. } \\
\text { Medicine added to the prescription: } \\
\text { Swarna vasant malti ras tab ( } 125 \mathrm{mg} \\
1 \mathrm{BD})\end{array}$ \\
\hline 6 & Sep 08 & $\begin{array}{l}\text { SpO2: } 96-99 \quad \text { PR: } 86-97 \\
\text { temp: } 98.4-99.7\end{array}$ & Mild fever and weakness reported. \\
\hline
\end{tabular}




\begin{tabular}{|c|c|c|c|}
\hline 7 & Sep 09 & $\begin{array}{l}\text { SpO2: } 96-98 \quad \text { PR: } 80-97 \\
\text { temp: } 97.7-98.6 \\
\text { RR (per min.): 19-24 }\end{array}$ & $\begin{array}{l}\text { No symptoms except mild weakness; } \\
\text { started indulging in physical activities } \\
\text { such as washing own clothes; respira- } \\
\text { tion rate observed to be slightly high } \\
\text { - practising Yoga Nidra and deep re- } \\
\text { laxation for it. }\end{array}$ \\
\hline 8 & Sep 10 & $\begin{array}{l}\text { SpO2: } 97-98 \quad \text { PR: } 82-90 \\
\text { temp: } 97.9-98.8 \\
\text { RR (per min.): } 20-26\end{array}$ & $\begin{array}{l}\text { No more weakness reported; new } \\
\text { symptoms emerged - dry cough and } \\
\text { breathlessness; HRCT chest done - } \\
\text { features suggestive of viral pneumonic } \\
\text { consolidation likely to be COVID-19, } \\
\text { CT severity score: } 2 \text {. }\end{array}$ \\
\hline 9 & Sep 11 & $\begin{array}{l}\text { SpO2: } 98-99 \quad \text { PR: } 73-84 \\
\text { temp: } 98.0-98.6 \\
\text { RR (per min.): } 20-23\end{array}$ & $\begin{array}{l}\text { Dry cough persists; SpO2 level normal } \\
\text { despite breathlessness, so lung capac- } \\
\text { ity observed to be preserved. }\end{array}$ \\
\hline 10 & Sep 12 & $\begin{array}{l}\text { SpO2: } 98-99 \quad \text { PR: } 68-87 \\
\text { temp: } 95.9 \\
\text { RR (per min.): } 21-25\end{array}$ & Improvement in dry cough. \\
\hline 11 & Sep 13 & $\begin{array}{l}\text { SpO2: } 98-99 \quad \text { PR: } 79-90 \\
\text { RR (per min.): } 20-24\end{array}$ & $\begin{array}{l}\text { Improvement in dry cough as well as } \\
\text { breathlessness, but talking for long } \\
\text { causes trouble. }\end{array}$ \\
\hline 12 & Sep 14 & $\begin{array}{l}\text { SpO2: } 97-99 \quad \text { PR: } 78-88 \\
\text { RR (per min.): } 22-24\end{array}$ & \\
\hline 13 & Sep 15 & & Discharged from hospital. \\
\hline 14 & Sep 16 & $\begin{array}{l}\text { SpO2: } 97-99 \quad \text { PR: } 80-90 \\
\text { temp: } 97.5-98.6\end{array}$ & $\begin{array}{l}\text { Talking for long still causes problem; } \\
\text { no new symptoms; } 90 \% \text { recovery re- } \\
\text { ported. }\end{array}$ \\
\hline
\end{tabular}

Table 15: Progress of patient P12 and evolution of the treatment plan

\section{Detailed treatment plan and progress tracking for P13:}

The patient presented with the following symptoms at the time of the first consultation: sore throat, body-ache, diarrhea and weakness. The patient also had 
mild cough with sputum.

History of the present illness was taken, which is as follows. The patient informed that he had a history of smoking until 2 years back, and had developed pulmonary tuberculosis a few months back; he also reported that mild cough with sputum had been recurring since then. After developing the initial symptoms, the patient, who had completed 6-months long treatment of pulmonary tuberculosis about one and a half months back, had visited the hospital on August 21, suspecting relapse of TB. Subsequently, he was prescribed the following medication for 5 days: Cap. Amoxycillin 500 mg (1 BD), Tab. Paracetamol 500 mg (SOS), Levocetrizine 5 mg (1 OD), Syp. Bromhexine (2 tsf BD). However, 2 days after the completion of the 5-day treatment, the patient developed fever again. As other symptoms started emerging, he got tested for COVID-19 on September 3, and was found positive. He had no fever at the time of the first consultation. He reported to have been taking Ayush Kwath on his own. He was also suggested the following Allopathic medication for fever: DOLO (SOS). Based on the patient's medical history and the symptoms presented, the following prescription for the Integrative Therapy was given: Ayurvedic medicines and procedures prescribed: Cap. Bowel Care (1TDS), Fifatrol (1TDS), Septalin (1BD) - all for 3 days; Sanshamani vati (1 BD), Laghu${ }_{1280}$ malini vasant (1 BD), Ayush Kwath (3gm BD), steam inhalation and gargles - for 15 days. Later, as specified in Table 16, he was additionally prescribed Vasavaleh, Syp. Jufex Forte during the course of the treatment.

Yoga Protocol: Sukshma Vyayama (upper and lower body parts); Breathing exercises (4 exercises with 5 iterations in each); asanas: Tadasana, TriyakTadasana, Trikonasana, Ardhachakrasana, Parshva Sukhasana, Utthana Mandukasana, Ardha Ustrasana, Vakrasana, Bhujangasana, Shalabhasana, Ardhahalasana, Pawanmuktasana, Shavasana; Pranayama (sectional breathing and full yogic breathing, Bhramari, Anulom-vilom, Udgeet); Dhyana.

The patient was almost fully compliant. He was regular in taking the medicines 1290 and attended almost all the yoga sessions. 
Details of the patient's progress and compliance during the course of the treatment are given below in Table 16 .

\begin{tabular}{|c|c|c|c|}
\hline Day & Date & Vital Measures & General Remarks \\
\hline 0 & Sep 04 & SpO2: $98 \quad$ PR: $81-89$ & $\begin{array}{l}\text { Symptoms reported: sore throat, } \\
\text { diarrhea, body-ache, weakness; } \\
\text { mild cough with sputum had been } \\
\text { persistent for a while }\end{array}$ \\
\hline 1 & Sep 05 & SpO2: 96-98 PR: 90-98 & Symptoms persist. \\
\hline 2 & Sep 06 & SpO2: 97-99 & $\begin{array}{l}\text { Relief from all symptoms except } \\
\text { weakness and mild cough with spu- } \\
\text { tum. }\end{array}$ \\
\hline 3 & Sep 07 & SpO2: 96-99 PR: 80-84 & Same as the previous day. \\
\hline 4 & Sep 08 & SpO2: $96-100 \quad$ PR: $75-87$ & Mild cough with sputum persists. \\
\hline 5 & Sep 09 & SpO2: 98-99 PR: 85-92 & $\begin{array}{l}\text { Patient reported } 90 \% \text { recovery, } \\
\text { though cough persists. }\end{array}$ \\
\hline 6 & Sep 10 & SpO2: 98-99 PR: $80-100$ & Same as above. \\
\hline 7 & Sep 11 & SpO2: $98 \quad$ PR: $90-100$ & Same as above. \\
\hline 8 & Sep 12 & SpO2: $96 \quad$ PR: $90-95$ & Same as above. \\
\hline 9 & Sep 13 & SpO2: $99-100$ & $\begin{array}{l}\text { Additional medicines prescribed: } \\
\text { Vasavaleh ( } 1 \text { tsf } \mathrm{BD}) \text {, Syp. Jufex } \\
\text { Forte ( } 2 \text { tsf TDS with lukewarm wa- } \\
\text { ter). }\end{array}$ \\
\hline 10 & Sep 14 & SpO2: 99-100 PR: 85 & Same as above. \\
\hline 11 & Sep 15 & SpO2: $98-100 \quad$ PR: $80-83$ & Same as above. \\
\hline 12 & Sep 16 & 83 & $\begin{array}{l}\text { The patient said that mild cough } \\
\text { had anyways been resurfacing often } \\
\text { owing to TB, and may have nothing } \\
\text { to do with COVID. He claimed to } \\
\text { be rejuvenated, have resumed nor- } \\
\text { mal appetite, and absence of any } \\
\text { weakness or tiredness; } 100 \% \text { recov- } \\
\text { ery reported. He also said he was } \\
\text { determined to continue with Yoga. }\end{array}$ \\
\hline
\end{tabular}




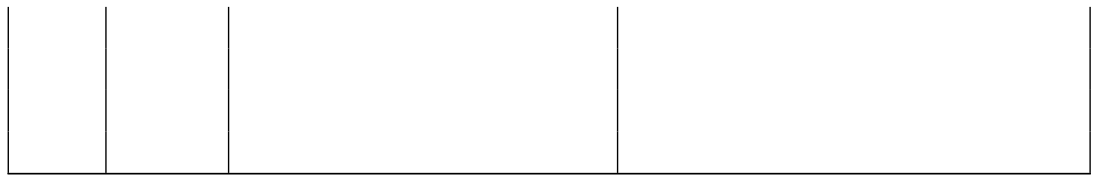

Table 16: Progress of patient P13 and evolution of the treatment plan

\section{Detailed treatment plan and progress tracking for P14:}

The patient presented with the following symptoms at the time of the first consultation: sore throat, body-ache, and cough.

History of the present illness was taken, which is as follows. The patient reported that he had fever the previous night and had taken DOLO 650 for the same. The patient was prescribed the following Ayurvedic medication for 2 days: Sudarshan Ghanvati (1 TDS), Sitopaladi Vati (1 TDS), Septilin tab. (1 BD), syp. Oritus (2 tsf TDS with lukewarm water). After taking the above medicines, the patient's symptoms no longer persisted. However, a few days later, when some of his family members started developing symptoms similar to COVID, the patient underwent RAT on September 7 and was tested positive. Subsequently, he was prescribed the following Allopathic supplements: Vitneurin CZS, Citravite XT.

The patient had been taking the following medicine regularly for his comorbidities: Remipress H 2.5 (1 OD), Reconia Silver, Ecosprin 150. He also reported sleeping difficulty since $4-5$ years.

Though none of the symptoms presented by the patient persisted by the time he was tested positive, based on the his medical history and age, the following prescription for the Integrative Therapy was given as a preventive measure:

15 Ayurvedic medicines and procedures prescribed: Sanshamani Vati (1 BD), Laghumalini Vasant (1 BD), Ayush Kwath (3gm BD), Chyawanprash, steam inhalation and gargles - all for 15 days.

Yoga Protocol: Breathing exercises (4 exercises with 5 iterations in each); Sukshma Vyayama (upper and lower body parts); asanas: TriyakTadasana, ${ }^{320}$ Trikonasana, Hastapadasana, Ardhachakrasana, Parshva Sukhasana, Sukhasana 
Twist, Utthana Mandukasana, Bhujangasana, Shalabhasana, Ardhahalasana, Pawanmuktasana, Shavasana; Pranayama (sectional breathing and full yogic breathing, Bhramari, Anulom-vilom, Udgeet); Dhyana.

The patient was almost fully compliant. He was regular in taking the medicines and attended almost all the yoga sessions. In a follow-up call after the treatment, he reported relief in his chronic sleeping problem with yoga nidra; he said he was able to sleep for 4-5 hrs. continuously, which was a remarkable relief to his previous condition when he used to have uninterrupted sleep for hardly 2-3 hrs.

Details of the patient's progress and compliance during the course of the treatment are given below in Table 17.

\begin{tabular}{|c|c|c|c|}
\hline Day & Date & Vital Measures & General Remarks \\
\hline 0 & Sep 01 & & $\begin{array}{l}\text { Symptoms: fever (previous night), } \\
\text { body-ache, sore throat, cough. }\end{array}$ \\
\hline 6 & Sep 07 & & $\begin{array}{l}\text { No symptoms at the time of enrol- } \\
\text { ment; vitals not shared }\end{array}$ \\
\hline 7 & Sep 08 & Temp: 99 & No symptoms reported. \\
\hline 8 & Sep 09 & SpO2: $98 \quad$ PR: 96 & No symptoms reported. \\
\hline 9 & Sep 10 & $\begin{array}{l}\text { SpO2: } 98-99 \quad \text { PR: } 93-95 \\
\text { temp: } 100\end{array}$ & $\begin{array}{l}\text { Reported fever; sleeping problem } \\
\text { aggravated since } 2 \text { days; also re- } \\
\text { ported blood in stools. Kwath dis- } \\
\text { continued by the doctor, coconut } \\
\text { water suggested; Yoga Nidra recom- } \\
\text { mended for the sleeping trouble. }\end{array}$ \\
\hline 10 & Sep 11 & $\begin{array}{l}\text { SpO2: } 97-98 \quad \text { PR: } 88-98 \\
\text { temp: } 93.9-96.1\end{array}$ & $\begin{array}{l}\text { No more fever or blood in stools; } \\
\text { sleeping difficulty continues. }\end{array}$ \\
\hline 11 & Sep 12 & $\begin{array}{l}\text { SpO2: } 96-99 \quad \text { PR: } 82-101 \\
\text { temp: } 94.7-95.9\end{array}$ & Same as the previous day. \\
\hline 12 & Sep 13 & $\begin{array}{l}\text { SpO2: } 99 \quad \text { PR: } 84-103 \\
\text { temp: } 96.0-96.5\end{array}$ & Same as above. \\
\hline
\end{tabular}




\begin{tabular}{|c|c|c|c|}
\hline 13 & Sep 14 & $\begin{array}{l}\text { SpO2: } 96-99 \quad \text { PR: } 80-98 \\
\text { temp: } 95.4\end{array}$ & Same as above. \\
\hline 14 & Sep 15 & $\begin{array}{l}\text { SpO2: } 97-99 \quad \text { PR: } 87-97 \\
\text { temp: } 95.2-95.4\end{array}$ & Same as above. \\
\hline 15 & Sep 16 & $\begin{array}{l}\text { SpO2: } 96 \quad \text { PR: } 100 \\
\text { temp: } 95\end{array}$ & $100 \%$ relief reported. \\
\hline 16 & Sep 17 & SpO2: $98 \quad$ PR: 92 & \\
\hline 17 & Sep 18 & $\begin{array}{l}\text { SpO2: } 97 \quad \text { PR: } 100 \\
\text { temp: } 95.9\end{array}$ & \\
\hline 18 & Sep 19 & SpO2: $98 \quad$ PR: 82 & \\
\hline
\end{tabular}

\section{Detailed treatment plan and progress tracking for P15:}

The patient presented with the following symptoms at the time of the first consultation: fever and cough.

History of the present illness was taken, which is as follows. Her symptoms had started appearing on September 5 with body temperature fluctuating between $101^{\circ} \mathrm{F}$ to $102^{\circ} \mathrm{F}$; the fever persisted for 2 days and thereafter surfaced on and off. She underwent RTPCR on September 8 and tested positive. The patient's family informed that she was allergic to Sulpha drugs, Fluoroquinalones and Tinidazole. Subsequently, she was prescribed PCM 650 (SOS). She joined the Integrative Therapy 2 days after her first consultation, i.e. on September 11, when she presented with the following symptoms: fever, cough, mild diarrhea, reduced appetite and weakness.

The patient had been taking the following medicines regularly for her comorbidities: Insulin, Thyroxine 50. Besides, she reported sleeping difficulty and a disturbed sleeping pattern since 5-6 years - tends to sleep around 2-3 a.m. and wakes up late at around 11 a.m. Also, occasional breathing problem was 
Details of the patient's progress and compliance during the course of the treatment are given below in Table 18 .

\begin{tabular}{|c|l|l|l|}
\hline Day & Date & Vital Measures & General Remarks \\
\hline \hline 0 & Sep 09 & & Symptoms: fever, cough. \\
\hline & & & $\begin{array}{l}\text { Symptoms: fever, cough, diarrhea (mild), re- } \\
\text { duced appetite and weakness; excessive sweat- } \\
\text { ing and scanty urination also reported; vitals } \\
\text { not shared; medicine Laxmivilas Ras replaced } \\
\text { with Septilin (1 BD) and Sitopaladi (1 TDS). }\end{array}$ \\
& Sep 11 & & \\
\hline
\end{tabular}




\begin{tabular}{|c|c|c|c|}
\hline 3 & Sep 12 & $\begin{array}{l}\text { SpO2: } 95-96 \\
\text { PR: } 82-91\end{array}$ & Symptoms persist (except fever). \\
\hline 4 & Sep 13 & $\begin{array}{l}\text { SpO2: } 95 \\
\text { PR: } 82\end{array}$ & $\begin{array}{l}\text { Overall about } 50 \% \text { relief reported by the pa- } \\
\text { tient; took coconut water to address the prob- } \\
\text { lem of scanty urination; advised to share blood } \\
\text { glucose level. }\end{array}$ \\
\hline 5 & Sep 14 & FBS: 135 & $\begin{array}{l}\text { Called the doctor to complain about upset } \\
\text { stomach and body-ache-Septilin replaced with } \\
\text { Bacnil ( } 1 \text { TDS) }\end{array}$ \\
\hline 6 & Sep 15 & $\begin{array}{l}\text { SpO2: } 94-95 \\
\text { PR: } 86-90 \\
\text { temp: } 98.0 \\
\text { FBS: } 100\end{array}$ & $\begin{array}{l}\text { Developed body-ache and felt feverish; relief } \\
\text { w.r.t. diarrhea (upset stomach) and reduced } \\
\text { appetite; sweating still more than usual. Bac- } \\
\text { nil taken only once. Patient not sharing blood } \\
\text { glucose level despite reminders. }\end{array}$ \\
\hline 7 & Sep 16 & RBS: 233 & $\begin{array}{l}\text { Weakness and mild cough persist, excessive } \\
\text { sweating and scanty urination continue; stom- } \\
\text { ach upset worsened again. Patient shared that } \\
\text { she has been practising Anulom Vilom and } \\
\text { Kapaal Bhati pranayama in the morning - } \\
\text { this was discussed with the Yoga therapist and } \\
\text { the patient was advised to discontinue Kapaal } \\
\text { Bhati. Resumed Bacnil at night due to wors- } \\
\text { ening of condition of stomach. }\end{array}$ \\
\hline 8 & Sep 17 & $\begin{array}{l}\text { SpO2: } 94 \\
\text { PR: } 82 \\
\text { FBS: } 113 \\
\text { BP: } 110 / 68\end{array}$ & \\
\hline 9 & Sep 18 & $\begin{array}{l}\text { SpO2: } 93 \\
\text { PR: } 97 \\
\text { temp: } 95.4\end{array}$ & $\begin{array}{l}\text { Relief in all symptoms except weakness and } \\
\text { mild cough. Oritus replaced with Koflet } \\
\text { syp. Patient discontinued Sanshamani Vati } \\
\text { and Laghumalini Vasant after taking once. }\end{array}$ \\
\hline 10 & Sep 19 & & $\begin{array}{l}\text { Symptoms same as above. Patient took a new } \\
\text { allopathic medicine (Canagliflozin 100mg) for } \\
\text { DM recommended by her physician; observed } \\
\text { improvement in scany urination problem. }\end{array}$ \\
\hline 11 & Sep 20 & & $\begin{array}{l}\text { Overall much better; weakness, mild cough and } \\
\text { mild stomach problem persist. }\end{array}$ \\
\hline
\end{tabular}




\begin{tabular}{|c|l|l|l|}
\hline 14 & Sep 23 & Temp: 100 & $\begin{array}{l}\text { Developed fever - took PCM; improvement in } \\
\text { all other symptoms. }\end{array}$ \\
\hline 15 & Sep 24 & & $\begin{array}{l}\text { Weakness still there; cough very very mild } \\
\text { (much improved); urination still not normal; } \\
\text { all other symptoms eliminated; overall 90\% re- } \\
\text { covery reported. }\end{array}$ \\
\hline
\end{tabular}

Table 18: Progress of patient P15 and evolution of the treatment plan

\section{Detailed treatment plan and progress tracking for P16:}

1375

The patient presented with the following symptoms at the time of the first consultation: body ache (legs,back) and sore throat, weakness.

History of the present illness was taken, which is as follows. The patient had developed initial symptoms (fever, headache, body-ache and burning in eyes) on 22 and tested positive. Subsequently, he was prescribed the following Allopathic medication: Erythromycin; he was also given the following supplements: Vitamin C, Evion 400 (Vitamin E), PCM 500, Zinc Sulphate, Thiamine Pyridoxine. Besides, at the time of the first consultation, the patient informed that he had been taking home made kadha (thrice a day) made with tulsi, adarak (ginger), dalchini (Cinnamon), sonth (dried ginger powder) and haldi (turmeric); besides, he had been doing steam inhalation (occasionally) and had done nasya twice till the first consultation.

The patient had been taking the following medicine regularly for his comorbidity: Glimepiride 1mg, Metformin 500mg, Neurobion forte (1 OD). He also reported weakness in memory since past 3 years.

Based on the patient's medical history and the symptoms presented, the following prescription for the Integrative Therapy was given:

Ayurvedic medicines and procedures prescribed: Sanshamani Vati (2 TDS), 
OD (replaced with Madhumeh Kusumakar Ras on patient's request), Nasya with Anu teil, steam inhalation, usha paan and gargles - all for 15 days.

Yoga Protocol: Sukshma Vyayama (upper and lower body parts); Breathing exercises (4 exercises with 5 iterations in each); asanas: Tadasana, Triyak-

Utthana Mandukasana, ArdhaUstrasana, Vakrasana, Bhujangasana, Shalabhasana, Ardhahalasana, Pawanmuktasana, Shavasana; Pranayama (sectional breathing and full yogic breathing, Bhramari, Anulom-vilom, Udgeet); Dhyana.

The patient was almost fully compliant. He was regular in taking the medicines and attended all the yoga sessions (except that it took him a while to arrange the medicine Madhumeh Kusumakar Ras, which he started taking on October 2).

Details of the patient's progress and compliance during the course of the treatment are given below in Table 19 .

\begin{tabular}{|c|c|c|c|}
\hline Day & Date & Vital Measures & General Remarks \\
\hline 0 & Sep 25 & $\begin{array}{l}\text { SpO2: } 97 \quad \text { PR: } 87-88 \\
\text { temp: } 96.4-97.5\end{array}$ & $\begin{array}{l}\text { Symptoms: body ache (mainly legs } \\
\text { and back), sore throat, and weakness. }\end{array}$ \\
\hline 1 & Sep 26 & $\begin{array}{l}\text { SpO2: } 96-98 \quad \text { PR: } 76-89 \\
\text { temp: } 96.3-96.8 \\
\text { FBS: } 142 \mathrm{mg} / \mathrm{dl}\end{array}$ & $\begin{array}{l}\text { Symptoms: body-ache, sore throat, } \\
\text { sputum, weakness. }\end{array}$ \\
\hline 2 & Sep 27 & PPBS: $184 \mathrm{mg} / \mathrm{dl}$ & $\begin{array}{l}\text { Same as before; patient expressed } \\
\text { concern about high blood glucose } \\
\text { level. }\end{array}$ \\
\hline 3 & Sep 28 & $\begin{array}{l}\text { SpO2: } 98 \quad \text { PR: } 81 \\
\text { temp: } 96.3 \\
\text { FBS: } 148 \mathrm{mg} / \mathrm{dl} ; \quad \text { PPBS: } \\
213 \mathrm{mg} / \mathrm{dl}\end{array}$ & $\begin{array}{l}\text { Improvement in body-ache and spu- } \\
\text { tum reported. Patient shared that the } \\
\text { kadha consumed the previous day had } \\
\text { gud, which could be the cause of sud- } \\
\text { den increase in blood glucose level; } \\
\text { requested replacement for the pre- } \\
\text { scribed medicine Basant Kusumakar } \\
\text { Ras. }\end{array}$ \\
\hline 4 & Sep 29 & $\begin{array}{l}\text { SpO2: } 98 \quad \text { PR: } 74-86 \\
\text { temp: } 96.5-96.8 \\
\text { FBS: } 198 \mathrm{mg} / \mathrm{dl} \\
\text { PPBS: } 162 \mathrm{mg} / \mathrm{dl}\end{array}$ & \\
\hline
\end{tabular}




\begin{tabular}{|c|c|c|c|}
\hline 5 & Sep 30 & $\begin{array}{l}\text { SpO2: } 97-98 \quad \text { PR: } 83-91 \\
\text { temp: } 96.2-97.6 \\
\text { FBS: } 146 \mathrm{mg} / \mathrm{dl} \\
\text { PPBS: } 152 \mathrm{mg} / \mathrm{dl}\end{array}$ & $\begin{array}{l}\text { Improvement in all symptoms: sore } \\
\text { throat - mild and occasional; sputum } \\
\text { improved (almost nil) }\end{array}$ \\
\hline 6 & Oct 01 & FBS: $145 \mathrm{mg} / \mathrm{dl}$ & $\begin{array}{l}\text { Symptoms same as above; overall } \\
80 \%-85 \% \text { relief reported. }\end{array}$ \\
\hline 7 & Oct 02 & $\begin{array}{l}\text { SpO2: } 98-99 \quad \text { PR: } 78-86 \\
\text { temp: } 96.3-96.5\end{array}$ & $\begin{array}{l}\text { Overall } 90 \% \text { relief reported; started } \\
\text { taking Madhumeh Kusumakar Ras }\end{array}$ \\
\hline 8 & Oct 03 & $\begin{array}{l}\text { SpO2: } 97-98 \quad \text { PR: } 76-83 \\
\text { temp: } 96.7-97.1\end{array}$ & $100 \%$ recovery reported. \\
\hline
\end{tabular}

Table 19: Progress of patient P16 and evolution of the treatment plan

The patient had been taking the following medicines regularly for his comorbidity: Istamet 50mg/1000mg (Matformin Hydrochloride), Amryl 2mg; he had 
also been taking neem+karela+jamun ras. He also had to take insulin after being tested positive with COVID. He also used to take insulin (need-based) to control his blood glucose level when required.

Based on the patient's medical history and the symptoms presented, the following prescription for the Integrative Therapy was given:

Ayurvedic medicines and procedures prescribed: Giloy Ghanvati, Basant Kusumkar Ras, Amalki Churna+Haldi Churna+Vijaysar Churna, nasya with anu teil -

1435 all for 15 days. He was also advised to do Usha paan, drink lukewarm water throughout the day and do steam inhalation.

Yoga Protocol: Sukshma Vyayama (upper and lower body parts); Breathing exercises (4 exercises with 5 iterations in each); asanas: Tadasana, TriyakTadasana, Trikonasana, Ardhachakrasana, Parshva Sukhasana, Utthana Mandukasana, ArdhaUstrasana, Vakrasana, Bhujangasana, Shalabhasana, Ardhahalasana, Pawanmuktasana, Shavasana; Pranayama (sectional breathing and full yogic breathing, Bhramari, Anulom-vilom, Udgeet); Dhyana.

The patient was almost fully compliant. He was regular in taking the medicines and attended all the yoga sessions. He expressed sincere desire to adopt Yoga in his lifestyle. He also consulted the doctor for long term management of diabetes.

Details of the patient's progress and compliance during the course of the treatment are given below in Table 20.

\begin{tabular}{|c|c|c|c|}
\hline Day & Date & Vital Measures & General Remarks \\
\hline 0 & Sep 28 & $\begin{array}{l}\text { SpO2: } 98-100 \quad \text { PR: } 91-96 \\
\text { temp: } 98.0-98.5 \\
\text { FBS: } 151 \mathrm{mg} / \mathrm{dl} \\
\text { RBS: } 200 \mathrm{mg} / \mathrm{dl}\end{array}$ & $\begin{array}{l}\text { Symptoms: anosmia, feeling cold, } \\
\text { feverish feeling in the evening, mild } \\
\text { weakness; also reported to have had } \\
\text { loose stools } 2-3 \text { times in the morning. }\end{array}$ \\
\hline 1 & Sep 29 & $\begin{array}{l}\text { SpO2: } 99-100 \quad \text { PR: } 92-93 \\
\text { temp: } 98.0-98.5 \\
\text { FBS: } 151 \mathrm{mg} / \mathrm{dl} \\
\text { PPBS: } 143 \mathrm{mg} / \mathrm{dl}\end{array}$ & $\begin{array}{l}\text { Symptoms persist; about } 10 \% \mathrm{im}- \\
\text { provement in anosmia. }\end{array}$ \\
\hline 2 & Sep 30 & $\begin{array}{l}\text { SpO2: } 98-100 \text { PR: } 92-104 \\
\text { temp: } 98.4 \\
\text { FBS: } 148 \mathrm{mg} / \mathrm{dl}\end{array}$ & $\begin{array}{l}\text { No more feverish feeling or feeling } \\
\text { cold; } 60 \% \text { improvement in anosmia. }\end{array}$ \\
\hline
\end{tabular}

PPBS: $143 \mathrm{mg} / \mathrm{dl}$ 


\begin{tabular}{|c|c|c|c|}
\hline 3 & Oct 01 & $\begin{array}{l}\text { SpO2: } 99-100 \quad \text { PR: } 97-104 \\
\text { temp: } 98.0-98.4 \\
\text { FBS: } 123 \mathrm{mg} / \mathrm{dl} ; \quad \text { PPBS: } \\
115 \mathrm{mg} / \mathrm{dl}\end{array}$ & Same as before. \\
\hline 4 & Oct 02 & $\begin{array}{l}\text { SpO2: } 99-100 \quad \text { PR: } 89-93 \\
\text { temp: } 97.8-98.0 \\
\text { FBS: } 142 \mathrm{mg} / \mathrm{dl} \\
\text { PPBS: } 135 \mathrm{mg} / \mathrm{dl}\end{array}$ & $\begin{array}{l}\text { No more symptoms - } 100 \% \text { recovery } \\
\text { reported. }\end{array}$ \\
\hline 5 & Oct 03 & $\begin{array}{l}\text { SpO2: } 100 \quad \text { PR: } 93-101 \\
\text { temp: } 98.0-98.1 \\
\text { FBS: } 125 \mathrm{mg} / \mathrm{dl}\end{array}$ & $\begin{array}{l}\text { Blood glucose level observed to be im- } \\
\text { proving - halved insulin dose } 2 \text { days } \\
\text { back, not taking insulin since the pre- } \\
\text { vious day }\end{array}$ \\
\hline 11 & Oct 09 & & $\begin{array}{l}\text { Follow-up call done - patient found } \\
\text { in good health; continuing medicines } \\
\text { and yoga practice. }\end{array}$ \\
\hline
\end{tabular}

Table 20: Progress of patient P17 and evolution of the treatment plan

\section{Detailed treatment plan and progress tracking for P18:}

The patient presented with the following symptoms at the time of the first consultation: fever, cough with sputum (chest congestion), breathlessness. Also, his SpO2 level during the day was 92. He was recommended Makarasana and Shithilasana; after practising both, the patient reported SpO2 level to be 98 in the evening.

History of the present illness was taken, which is as follows. The patient started feeling unwell and feverish on September 24, following which he developed fever, and throat and chest congestion on September 26. Thereafter, he underwent RTPCR on September 28 and was tested positive. Subsequently, he was prescribed the following allopathic medicines: tab. Augmentin (1 BD for 5 days); tab. Deriphyllin 150 (1 TDS), Dolo 650 (1 TDS) - both for 3 days. He was also prescribed the following supplements: Vitneurin CZS (1 OD), tab. Cit- 
ravite XT (1 OD) - both for 15 days. Besides, the patient also started doing self-nebulization owing to his seasonal bronchitis since childhood. The patient had been taking the following medicines regularly for his comorbidities: Telmikind. Also, he has been keeping Asthma under control by self nebulization at the onset of premonitory symptoms of congestion - has not had any bronchial spasm since 15 years.

Based on the patient's medical history and the symptoms presented, the following prescription for the Integrative Therapy was given:

Ayurvedic medicines and procedures prescribed: cap. Astha 15 (3 days); syp. Astha 15, Sanshamani Vati, Laghumalini Vasant, Chyawnprash, Ayush Kwath, steam inhalation and gargles - all for 15 days.

Yoga Protocol: Sukshma Vyayama (upper and lower body parts - could not do wrist rotation); Breathing exercises (3 exercises with 5 iterations in each); asanas: Tadasana, TriyakTadasana, Trikonasana, Ardhachakrasana, Parshva Sukhasana, Utthana Mandukasana (performed in Sukhasana), ArdhaUstrasana, Vakrasana (unable to do), Shavasana; Pranayama (Bhramari, Anulom-vilom, Udgeet); Dhyana. The patient was almost fully compliant with regards to the medicines, but was not willing to take the yoga sessions. He regularly took all the medicines except Kwath; he discontinued yoga sessions after a few days (even the first few days were attended off and on in a reluctant way). In the few sessions that he attended the therapist observed that he found it difficult to follow the instructions. Therefore, the recorded video (supposed to be shared with him) was not shared with him.

Details of the patient's progress and compliance during the course of the treatment are given below in Table 21 .

\begin{tabular}{|c|c|l|l|}
\hline Day & Date & Vital Measures & General Remarks \\
\hline \hline 0 & Sep 29 & & $\begin{array}{l}\text { Symptoms: fever, cough with sputum, } \\
\text { chest congestion and breathlessness. }\end{array}$ \\
\hline 1 & Sep 30 & $\begin{array}{l}\text { SpO2: } 98 \quad \text { PR: } 75-85 \\
\text { temp: } 98.4-100.3\end{array} \quad \begin{array}{l}\text { Symptoms: fever, body-ache, weak- } \\
\text { ness, sore throat, cough with sputum } \\
\text { and reduced appetite. }\end{array}$ \\
& & & 94
\end{tabular}




\begin{tabular}{|c|c|c|c|}
\hline 2 & Oct 01 & $\begin{array}{l}\text { SpO2: } 98 \quad \text { PR: } 62-88 \\
\text { temp: } 97.0-98.0 \\
\text { BP: } 106 / 69\end{array}$ & $\begin{array}{l}\text { No fever or body-ache, improvement } \\
\text { in weakness, cough and appetite; pa- } \\
\text { tient reported to be feeling overall } \\
\text { better; however, he reported to have } \\
\text { lost } 3 \mathrm{Kgs} \text { in the past } 15 \text { days. }\end{array}$ \\
\hline 3 & Oct 02 & $\begin{array}{l}\text { SpO2: } 98-99 \quad \text { PR: } 65-86 \\
\text { temp: } 96.9-98.1 \\
\text { BP sys: } 115-125 \text { dias: } 78\end{array}$ & $\begin{array}{l}\text { Patient reported to have felt tired af- } \\
\text { ter the yoga session; BP observed to } \\
\text { be on lower side; advised to discon- } \\
\text { tinue BP medicine for } 2 \text { days; patient } \\
\text { advised to avoid cold things to eat; } \\
\text { appetite getting normal. }\end{array}$ \\
\hline 4 & Oct 03 & $\begin{array}{l}\text { SpO2: } 98 \text { PR: } 73-78 \\
\text { temp: } 97.6-97.9 \\
\text { BP sys: } 107-126 \\
\text { BP dias: } 74-93\end{array}$ & $\begin{array}{l}\text { Continuing improvement in weak- } \\
\text { ness, thick expectoration (steam in- } \\
\text { halation advised), appetite normal; } \\
\text { overall } 60 \%-70 \% \text { recovery reported; } \\
\text { slight drowsiness after yoga session re- } \\
\text { ported. }\end{array}$ \\
\hline 5 & Oct 04 & $\begin{array}{l}\text { SpO2: } 98 \quad \text { PR: } 74-76 \\
\text { temp: } 97.5-98.1 \\
\text { BP sys: } 114-127 \\
\text { BP dias: } 76-88\end{array}$ & Symptoms same as before. \\
\hline 6 & Oct 05 & $\begin{array}{l}\text { SpO2: } 98 \text { PR: } 75-86 \\
\text { temp: } 97.1-98.2 \\
\text { BP sys: } 114-129 \\
\text { BP dias: } 76-86\end{array}$ & $\begin{array}{l}\text { Continuing improvement in weakness; } \\
\text { thick expectoration (taking steam } \\
\text { with ajwain water); BP medicine still } \\
\text { discontinued - patient feeling fine. }\end{array}$ \\
\hline 7 & Oct 06 & $\begin{array}{l}\text { SpO2: } 97-98 \quad \text { PR: } 76-86 \\
\text { temp: } 97.4-98.3 \\
\text { BP sys: } 110-119 \\
\text { BP dias: } 69-86\end{array}$ & $\begin{array}{l}\text { patient in good health; continuing } \\
\text { medicines. }\end{array}$ \\
\hline \multirow[t]{2}{*}{10} & \multirow[t]{2}{*}{ Oct 09} & $\begin{array}{l}\text { SpO2: } 97-98 \quad \text { PR: } 76-86 \\
\text { temp: } 97.4-98.3 \\
\text { BP sys: } 110-119\end{array}$ & $\begin{array}{l}\text { patient in good health and reported } \\
\text { to have almost recovered (except mild } \\
\text { expectoration); continuing medicines; }\end{array}$ \\
\hline & & BP dias: $69-86$ & BP medicine still discontinued. \\
\hline
\end{tabular}




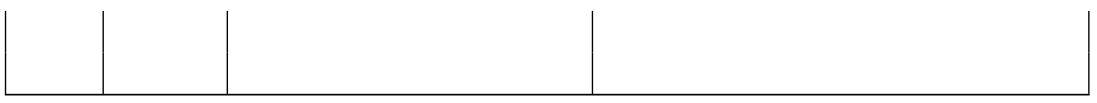

Table 21: Progress of patient P18 and evolution of the treatment plan

\section{Detailed treatment plan and progress tracking for P19:}

The patient was asymptomatic at the time of the first consultation.

History of the present illness was taken, which is as follows. The patient underwent RTPCR on October 2 due to contact history with a positive case, and was himself found positive. Subsequently, he was prescribed the following Allopathic medicines: Azithromycin, Vitamin C, Zinc -all for 5 days. He also reported to be taking the following Homeopathic medicine: Immunomodulator (thrice per month for 3 months; already taken for 2 months). Besides, he was taking home made kadha prepared with kali mirch (black pepper), laung (cloves), tej patta (bay leaves), ilaichi (cardamom), dalchini (cinnamon), haldi (turmeric) + lemon The patient had been taking the following medicines regularly for his comorbidities: Telma-40mg and a blood thinner. Besides other comorbidities, the patient also reported dyspnea on exertion as a persistent problem.

Based on the patient's medical history, the following prescription for the Integrative Therapy was given as a preventive measure:

Ayurvedic medicines and procedures prescribed: Sanshamani Vati, Ashwagandha Vati, nasya with Anu teil. He was also advised to do usha paan, steam inhalation, gargles and drink lukewarm water throughout the day.

Yoga Protocol: Breathing exercises (3 exercises with 5 iterations in each); Sukshma Vyayama (upper and lower body parts); asanas: Parshva Sukhasana, Sukhasana Twist, Utthana Mandukasana, Ardhahalasana with folded leg, Shavasana; Pranayama (Anulom-vilom, Udgeet); Dhyana.

The patient was only partly compliant with respect to the medicines, but was very enthusiastic about the yoga sessions. Out of the medicines and procedures prescribed, he regularly took Ashwagandha Vati and did nasya. He was regular in attending yoga sessions, and very diligently continued self-practice after 
the guided sessions ended. During follow-up calls, he several times reported that his lungs were functioning much better and his breath holding capacity had improved with the practice of pranayama. He sounded very energetic and rejuvenated by the end of the treatment; he was particularly excited about pranayama and had started regularly practising it.

The patient remained asymptomatic throughout. He did not share his vitals regularly; instead, he informed the usual range of observations as follows: $\mathrm{SpO} 2$ : 97-98; PR: 60-70; BP: 120/70. Also, he informed that the temperature remained normal. At the end of the isolation period, and the completion of the preventive treatment administered, the patient was prescribed the following for long term use based on his medical condition: Sanshamani Vati (2 BD after meal), Kalmegh Vati (1 BD after meal).

\section{Detailed treatment plan and progress tracking for P20:}

The patient presented with the following symptoms at the time of the first consultation: fever, body-ache and weakness.

History of the present illness was taken, which is as follows. The patient had started feeling unwell on October 04, with temperature around $99^{\circ} \mathrm{F}-100^{\circ} \mathrm{F}$, for which she took PCM. Thereafter, she remained afebrile for the next 3 days. However, the fever relapsed on October 8, following which she consulted the doctor on October 10 and was given the following Ayurvedic prescription for 2 days: Sudarshan Ghanvati (1 TDS), Amlapittantak Yog (1 TDS). She was also suggested to take PCM (SOS). She underwent the RTPCR on October 11 and was tested positive. Subsequently, she was prescribed the following Allopathic medicines: Azee 500 (1 OD for 3 days), PCM (SOS), Vitneurin CZS (1 OD for 15 days), Citranite XT (1 OD for 15 days).

${ }_{1545}$ The patient had been taking the following medicines regularly for her comorbidities: Amlodipine. She also reported emotional disturbance and disturbed sleep since March 2020 ever since loss of parent-in-law; tends to wake up every 
couple of hours.

Based on the patient's medical history and the symptoms presented, the following prescription for the Integrative Therapy was given:

Ayurvedic medicines and procedures prescribed: Sudarshan Ghanvati (1 TDS) and Amlapittantak Yog (1 TDS) for 3 days; Sanshamani Vati (1 BD), Laghumalini Vasant (1 BD), Ayush Kwath (BD), steam inhalation and gargles - all for 15 days.

1555 Yoga Protocol: Sukshma Vyayama (upper and lower body parts); Breathing exercises (4 exercises with 5 iterations in each); asanas: Tadasana, TriyakTadasana, Trikonasana, Ardhachakrasana, Parshva Sukhasana, Utthana Mandukasana, ArdhaUstrasana, Vakrasana, Bhujangasana, Shalabhasana, Ardhahalasana, Pawanmuktasana, Shavasana; Pranayama (sectional breathing and full yogic breathing, Bhramari, Anulom-vilom, Udgeet); Dhyana.

The patient was almost fully compliant. She was regular in taking all the medicines and attended most of the yoga sessions. Also, she mentioned several times during the course of the treatment that she was being immensely benefited by yoga nidra with respect to her sleeping trouble.

Details of the patient's progress and compliance during the course of the treatment are given below in Table 22 .

\begin{tabular}{|c|c|c|c|}
\hline Day & Date & $\begin{array}{l}\text { Vital Mea- } \\
\text { sures }\end{array}$ & General Remarks \\
\hline 0 & Oct 10 & & Symptoms: fever, body-ache, weakness. \\
\hline 2 & Oct 12 & & $\begin{array}{l}\text { Symptoms: body-ache, weakness, mild sore } \\
\text { throat, heaviness in abdomen. }\end{array}$ \\
\hline 3 & Oct 13 & $\begin{array}{l}\text { SpO2: } 96-97 \\
\text { PR: } 66-76 \\
\text { Temp: } 98.5-98.6 \\
\text { BP sys: } 118-128 \\
\text { BP dias: } 71-82\end{array}$ & $\begin{array}{l}\text { Improvement in body-ache; other symptoms } \\
\text { persist. }\end{array}$ \\
\hline 4 & Oct 14 & $\begin{array}{l}\text { SpO2: } 98-99 \\
\text { PR: } 63-76 \\
\text { Temp: } 98.5-98.8 \\
\text { BP sys: } 118 / 78\end{array}$ & $\begin{array}{l}\text { Body-ache, sore throat persist; improvement in } \\
8^{\text {weakness; overall feeling better than the previ- }} \\
\text { ous day. }\end{array}$ \\
\hline
\end{tabular}




\begin{tabular}{|c|c|c|c|}
\hline 5 & Oct 15 & $\begin{array}{l}\text { SpO2: } 97-98 \\
\text { PR: } 79-86 \\
\text { Temp: } 98.6-98.7 \\
\text { BP sys: } 134 / 91\end{array}$ & $\begin{array}{l}\text { Body-ache and weakness persist; sore throat im- } \\
\text { proved (very mild now); patient reported numb- } \\
\text { ness in hands and a lot of pain in the feet the } \\
\text { previous night. She said it was as if the pain } \\
\text { is rotating and affecting different body parts. } \\
\text { Also, reported to be getting tired after daily } \\
\text { chores and feeling breathlessness after talking } \\
\text { for long. }\end{array}$ \\
\hline 6 & Oct 16 & $\begin{array}{l}\text { SpO2: } 96 \\
\text { PR: } 73-78 \\
\text { Temp: } 97.9-98.6 \\
\text { BP: } 128 / 79\end{array}$ & $\begin{array}{l}\text { Body-ache, sore throat and weakness per- } \\
\text { sist; developed headache and constipation (new } \\
\text { symptoms). Patient informed about extreme } \\
\text { restlessness last night - had called the doctor at } \\
1 \text { : } 00 \text { a.m. due to severe headache and anx- } \\
\text { iety; patient took Disprin on her own. Doc- } \\
\text { tor assessed this to be gastric trouble due to } \\
\text { improper dinner and suggested green tea. Pa- } \\
\text { tient also reported sleeplessness, and breathless- } \\
\text { ness after talking for long. Yoga nidra rec- } \\
\text { ommended for sleep; papaya recommended for } \\
\text { stomach; Makarasana and Shithilasana sug- } \\
\text { gested for breathlessness; steam inhalation also } \\
\text { suggested. }\end{array}$ \\
\hline 7 & Oct 17 & $\begin{array}{l}\text { SpO2: } 96-97 \\
\text { PR: } 73-82 \\
\text { Temp: } 98.8 \\
\text { BP: } 110 / 71\end{array}$ & $\begin{array}{l}\text { Weakness, breathlessness and constipation per- } \\
\text { sist despite eating papaya and doing steam in- } \\
\text { halation; a lot of relief reported in sleeping trou- } \\
\text { ble due to yoga nidra }\end{array}$ \\
\hline 8 & Oct 18 & $\begin{array}{l}\text { SpO2: } 98 \\
\text { PR: } 75 \\
\text { temp: } 98.3 \\
\text { BP: } 118 / 75\end{array}$ & $\begin{array}{l}\text { Weakness persists - felt weak in the morning, } \\
\text { but improved by the evening. No more con- } \\
\text { stipation. Yoga nidra done again - found very } \\
\text { helpful. }\end{array}$ \\
\hline 9 & Oct 19 & $\begin{array}{l}\text { SpO2: } 97 \\
\text { PR: } 90 \\
\text { Temp: } 98.8 \\
\text { BP: } 120 / 74\end{array}$ & $\begin{array}{l}\text { Weakness persists, but a lot of overall improve- } \\
\text { ment reported. }\end{array}$ \\
\hline 10 & Oct 20 & $\begin{array}{l}\text { SpO2: } 96 \\
\text { PR: } 65 \\
\text { Temp: } 97.8 \\
\text { BP: } 128 / 84\end{array}$ & $\begin{array}{l}75 \%-80 \% \text { recovery reported; however weakness } \\
\text { persists - patient reported pain in right hand } \\
\text { after writing. }\end{array}$ \\
\hline
\end{tabular}




\begin{tabular}{|l|l|l|l|} 
& & & \\
11 & Oct 21 & $\begin{array}{l}\text { SpO2: } 98 \\
\text { PR: } 78 \\
\text { Temp: } 98.2 \\
\text { BP: } 123 / 70\end{array}$ & \\
\hline 12 & Oct 22 & $\begin{array}{l}\text { PR: } 74 \\
\text { Temp: } 98.6 \\
\text { BP: } 125 / 77\end{array}$ & $\begin{array}{l}\text { Patient reported almost complete re- } \\
\text { covery, though still experiencing very } \\
\text { mild weakness; felt fit enough to re- } \\
\text { sume household work. }\end{array}$ \\
\hline
\end{tabular}

Table 22: Progress of patient P20 and evolution of the treatment plan

\section{Detailed treatment plan and progress tracking for P21:}

1570

The patient presented with the following symptoms at the time of the first consultation: dry cough, and bad taste (which he attributed to the medicines). He also reported that he used to experience dry mouth and muscle cramps with increase in blood glucose level. started developing the initial symptoms (sore throat/dryness in throat, bodyache) on October 14, following which he underwent RAT on October 15, and was tested positive. Subsequently, he was prescribed the following Allopathic medicines for 5 days: PCM 500, Doxy 400, LCZ, Vit C, Azithromycin 500, kadha prepared with dalchini (cinnamon), adrak (ginger), methi and curry patta for the past one month.

The patient had been taking the following medicines regularly for his comorbidities: Giolife 12G, Inventia (metformin). The patient also reported that nearly one and a half months back, his HbA1c was 10\%, and that his FBS used to be around $200 \mathrm{mg} / \mathrm{dl}$; however, after he started taking kadha, FBS had been around $140 \mathrm{mg} / \mathrm{dl}$.

Based on the patient's medical history and the symptoms presented, the follow- 
ing prescription for the Integrative Therapy was given:

Ayurvedic medicines and procedures prescribed: Pathyadi kadha, Sanshamani

Vati, Diabecon, nasya with til oil, usha paan, steam inhalation and gargles - all for 15 days; some modification was made to the prescription during the course of treatment based on the condition of the patient.

Yoga Protocol: Sukshma Vyayama (upper and lower body parts); Breathing exercises (4 exercises with 5 iterations in each); asanas: Tadasana, TriyakTadasana, Trikonasana, Hastapadasana, Ardhachakrasana, Parshva Sukhasana, Utthana Mandukasana, ArdhaUstrasana, Vakrasana, Bhujangasana, Shalabhasana, Ardhahalasana, Pawanmuktasana, Shavasana; Pranayama (sectional breathing and full yogic breathing, Bhramari, Anulom-vilom, Udgeet); Dhyana.

The patient was almost fully compliant. He was regular in taking all the medicines and attended all the yoga sessions.

Details of the patient's progress and compliance during the course of the treatment are given below in Table 23 .

\begin{tabular}{|c|c|c|c|}
\hline Day & Date & Vital Measures & General Remarks \\
\hline 0 & Oct 17 & $\begin{array}{l}\text { SpO2: } 97-98 \quad \text { PR: } 83-90 \\
\text { Temp: } 95.5\end{array}$ & Symptoms: dry cough, bad taste. \\
\hline 1 & Oct 18 & $\begin{array}{l}\text { SpO2: } 97 \quad \text { PR: } 89 \\
\text { Temp: } 96 .\end{array}$ & Symptoms persist. \\
\hline 2 & Oct 19 & $\begin{array}{ll}\text { SpO2: } 97 & \text { PR: } 90 \\
\text { FBS: } 190 & \text { PPBS:300 }\end{array}$ & $\begin{array}{l}\text { Symptoms same as before; blood glu- } \\
\text { cose level observed to be high. }\end{array}$ \\
\hline 3 & Oct 20 & $\begin{array}{l}\text { SpO2: } 98 \quad \text { PR: } 89 \\
\text { Temp: } 95.4\end{array}$ & \\
\hline 4 & Oct 21 & $\begin{array}{l}\text { SpO2: } 98 \quad \text { PR: } 86 \\
\text { Temp: } 94.5\end{array}$ & \\
\hline 5 & Oct 22 & $\begin{array}{l}\text { SpO2: } 97 \quad \text { PR: } 95 \\
\text { Temp: } 94.5 \quad \text { RBS: } 250\end{array}$ & $100 \%$ recovery reported. \\
\hline 6 & Oct 23 & $\begin{array}{ll}\text { SpO2: } 97 & \text { PR: } 88 \\
\text { Temp: } 95.5 & \\
\text { FBS: } 200 & \text { PPBS: } 280\end{array}$ & $\begin{array}{l}\text { Blood glucose level observed to be } \\
\text { high. }\end{array}$ \\
\hline
\end{tabular}




\begin{tabular}{|c|c|c|c|c|}
\hline 7 & Oct 24 & $\begin{array}{l}\text { SpO2: } 97 \\
\text { Temp: } 95.2 \\
\text { FBS: } 203\end{array}$ & $\begin{array}{l}\text { PR: } 98 \\
\text { PPBS: } 280\end{array}$ & $\begin{array}{l}\text { The dosage of Diabecon doubled } \\
\text { hereon to address the high levels of } \\
\text { blood glucose. }\end{array}$ \\
\hline 8 & Oct 25 & $\begin{array}{l}\text { SpO2: } 97 \\
\text { Temp: } 96.0 \\
\text { FBS: } 168\end{array}$ & $\begin{array}{l}\text { PR: } 91 \\
\text { PPBS: } 348\end{array}$ & \\
\hline 9 & Oct 26 & $\begin{array}{l}\text { SpO2: } 98 \\
\text { Temp: } 95.4 \\
\text { FBS: } 204\end{array}$ & $\begin{array}{l}\text { PR: } 96 \\
\text { PPBS: } 305\end{array}$ & \\
\hline 10 & Oct 27 & FBS: 170 & PPBS: 255 & \\
\hline 13 & Oct 30 & FBS: 152 & RBS: 258 & $\begin{array}{l}\text { Blood glucose level appears to be re- } \\
\text { ducing. }\end{array}$ \\
\hline 14 & Nov 01 & FBS: 125 & PPBS: 240 & $\begin{array}{l}\text { Blood glucose level appears to be re- } \\
\text { ducing.. }\end{array}$ \\
\hline
\end{tabular}

Table 23: Progress of patient P21 and evolution of the treatment plan

\section{Detailed treatment plan and progress tracking for P22:}

The patient presented with the following symptoms at the time of the first consultation: fever, body-ache, weakness, mild cough, reduced appetite (reported to be taking hardly any solid food since many days). Also, his SpO2 level was

History of the present illness was taken, which is as follows. The patient started feeling unwell and feverish on October 17 with temperature around $99^{\circ} \mathrm{F}$ to $100^{\circ} \mathrm{F}$. Following this, he was prescribed Allopathic medication for 5 days, no records of which are available. When the symptoms persisted,the patient underwent RTPCR on October 21 and was tested positive. Thereafter, he was prescribed the following Allopathic medicines: tab. Ivermectol 12mg (1 OD for 3 days), tab. Zathrin 500 (1 OD for 7 days, though he took only for 5 days), Meftal Forte (SOS) - which was changed to DOLO 650 (SOS) on Oct 26; he 
was also given the following supplements: Limcee 500 (1 BD), Vitneurin CZS

1620 (1 OD).

The patient was reported not to have any comorbidities, though his blood glucose levels remained at borderline (as informed by the patient). However, high blood glucose levels were observed during COVID. The patient was advised to be cautious about eating habits. Also, he was suggested to get tested after recovery from COVID so as to rule out the possibility of temporary onset of DM with COVID. After recovery, the patient underwent another blood test which revealed high blood glucose levels. The patient sought Ayurvedic treatment for the same; he was duly provided consultation.

Based on the patient's medical history and the symptoms presented, the follow1630 ing prescription for the Integrative Therapy was given: Ayurvedic medicines and procedures prescribed: Sanshamani Vati (1 BD), Laghumalini Vasant (1 BD), Chyawanprash (1 tsf. daily with milk), Ayush Kwath (BD), steam inhalation and gargles - all for 15 days. Some more medicines, viz. Sudarshan Ghanvati, Laxmivilas Ras, Ashwagandha Vati, Himcocid, Amlapittantak yog, tab. Sitopaladi were progressively added to the prescription based on the condition of the patient; the details of the same have been furnished in Table 24 Yoga Protocol: Sukshma Vyayama (upper and lower body parts); Breathing exercises (4 exercises with 5 iterations in each); asanas: Tadasana, Triyak-

${ }_{1640}$ Tadasana, Trikonasana, Hastapadasana, Ardhachakrasana, Parshva Sukhasana, Utthana Mandukasana, ArdhaUstrasana, Vakrasana, Bhujangasana, Shalabhasana, Ardhahalasana, Pawanmuktasana, Shavasana; Pranayama (sectional breathing and full yogic breathing, Bhramari, Anulom-vilom, Udgeet); Dhyana.

The patient was almost fully compliant. He was regular in taking all the 1645 medicines and attended most of the yoga sessions.

Details of the patient's progress and compliance during the course of the treatment are given below in Table 24 . 


\begin{tabular}{|c|c|c|c|}
\hline Day & Date & Vital Measures & General Remarks \\
\hline 0 & Oct 22 & $\begin{array}{l}\text { SpO2: } 94-97 \\
\text { Temp: } 99.0-100.0\end{array}$ & $\begin{array}{l}\text { Symptoms: fever, body-ache, weak- } \\
\text { ness, mild cough, reduced appetite } \\
\text { (hardly taking any solid food); SpO2 } \\
\text { below } 95 \text { - Makarasana and Shithi- } \\
\text { lasana recommended. }\end{array}$ \\
\hline 1 & Oct 23 & $\begin{array}{l}\text { SpO2: } 93-96 \\
\text { Temp: } 98.3-102.0\end{array}$ & $\begin{array}{l}\text { Symptoms persist; still not eating } \\
\text { anything solid. }\end{array}$ \\
\hline 2 & Oct 24 & $\begin{array}{l}\text { SpO2: } 92-96 \\
\text { Temp:98.3-101.0 }\end{array}$ & $\begin{array}{l}\text { Symptoms same as before; fever ac- } \\
\text { companied with cold feeling; SpO } 2 \\
\text { observed to be } 92 \text { in the morning, } \\
\text { later rose to } 94 \text { and then } 96 \text {. }\end{array}$ \\
\hline 3 & Oct 25 & $\begin{array}{l}\text { SpO2: } 95-96 \quad \text { PR: } 77-85 \\
\text { Temp: } 98.0-100.0\end{array}$ & $\begin{array}{l}\text { Fever persists with cold feeling; no } \\
\text { more body-ache; appetite still very } \\
\text { poor. }\end{array}$ \\
\hline 4 & Oct 26 & $\begin{array}{l}\text { SpO2: } 94-96 \quad \text { PR: } 72-88 \\
\text { Temp: } 98.0-100.0 \\
\text { FBS: } 149 \quad \text { PPBS: } 251\end{array}$ & $\begin{array}{l}\text { Condition same as before; tiredness } \\
\text { and weakness worsened. Blood test } \\
\text { done at the suggestion of doctor - } \\
\text { blood glucose level found to be high. } \\
\text { Following medicines added to the pre- } \\
\text { scription: Sudarshan Ghanvati ( } 1 \\
\text { TDS), Laxmivilas Ras (1 TDS) - for } \\
3 \text { days; Ashwagandha Vati (1 BD for } \\
15 \text { days) }\end{array}$ \\
\hline 5 & Oct 27 & $\begin{array}{l}\text { SpO2: } 95-97 \quad \text { PR: } 77-88 \\
\text { Temp: } 99.0-101.0\end{array}$ & $\begin{array}{l}\text { Vomited in the morning - felt light af- } \\
\text { ter that and ate solid food after many } \\
\text { days. Fever, weakness, mild cough } \\
\text { and reduced appetite persist. }\end{array}$ \\
\hline 6 & Oct 28 & $\begin{array}{l}\text { SpO2: } 94-95 \quad \text { PR: } 77-86 \\
\text { Temp: } 99.0-100.3\end{array}$ & $\begin{array}{l}\text { Heaviness in stomach - could hardly } \\
\text { eat anything; taste of mouth changed } \\
\text { - found food very salty; coughing } \\
\text { slightly increased - syp. Koflet SF } \\
\text { given. }\end{array}$ \\
\hline 7 & Oct 29 & $\begin{array}{l}\text { SpO2: } 92-95 \quad \text { PR: } 71-88 \\
\text { Temp: } 98.5-102.0\end{array}$ & $\begin{array}{l}\text { Fever in the morning and at night, } \\
\text { not during the day ( } 99.5 \text { at } 10: 00 \\
\text { p.m.) - no medicine taken for fever } \\
\text { after morning; slight improvement in } \\
\text { appetite. ; Himcocid ( } 2 \text { tsp. TDS), } \\
\text { Amlapittantak yog, tab. Sitopaladi } \\
\text { added to prescription. }\end{array}$ \\
\hline
\end{tabular}




\begin{tabular}{|c|c|c|c|}
\hline 8 & Oct 30 & $\begin{array}{l}\text { SpO2: } 95-96 \quad \text { PR: } 71-81 \\
\text { Temp: } 98.0-98.5\end{array}$ & $\begin{array}{l}\text { No fever; improvement in weakness; } \\
\text { ate proper food; mild cough persists. } \\
\text { Overall } 70 \% \text { recovery reported. }\end{array}$ \\
\hline 9 & Oct 31 & $\begin{array}{l}\text { SpO2: } 97-98 \quad \text { PR: } 68-73 \\
\text { Temp: } 97.0-97.3\end{array}$ & \\
\hline 10 & Nov 01 & & $\begin{array}{l}\text { Complete recovery reported with nor- } \\
\text { mal appetite. }\end{array}$ \\
\hline 11 & Nov 02 & $\begin{array}{l}\text { SpO2: } 97-98 \quad \text { PR: } 70-81 \\
\text { Temp: } 97.0-99.0\end{array}$ & \\
\hline 14 & Nov 05 & & $\begin{array}{l}\text { Patient found in good health during } \\
\text { follow-up. }\end{array}$ \\
\hline
\end{tabular}

Table 24: Progress of patient P22 and evolution of the treatment plan

\section{Detailed treatment plan and progress tracking for P23:}

The patient presented with the following symptoms at the time of the first consultation: mild cough, body ache, weakness.

History of the present illness was taken, which is as follows. The patient started developing initial symptoms around October 21-22. She underwent RTPCR on October 23, and was tested positive, following which she was suggested to take PCM SOS. Subsequently, she joined the Integrative Therapy.

The patient was reported not to be taking any medicines regularly. The only chronic problem she had was seasonal bronchitis, which she had been managing with steam and herbal concoctions.

Based on the patient's medical history and the symptoms presented, the following prescription for the Integrative Therapy was given:

Ayurvedic medicines and procedures prescribed: Sanshamani Vati (1 BD), 
Laghumalini Vasant (1 BD), Ayush Kwath (BD), Chyawanprash (1 tsf. daily with milk), steam inhalation and gargles - all for 15 days. The following medicines were added to the prescription within two days: Sudarshan Ghanvati (1 TDS), Astha-15 (1 BD), Bresol (1 BD), syp. Astha-15 (2 tsf. TDS with lukewarm water) - to be taken for 3 days, and Ashwagandha Vati to be taken for 15 days; the details of the same have been furnished in Table 25 Yoga Protocol: Sukshma Vyayama (upper and lower body parts); Breathing exercises (4 exercises with 5 iterations in each); asanas: Tadasana, TriyakTadasana, Trikonasana, Hastapadasana, Ardhachakrasana, Parshva Sukhasana, Utthana Mandukasana, ArdhaUstrasana, Vakrasana, Bhujangasana, Shalabhasana, Ardhahalasana, Pawanmuktasana, Shavasana; Pranayama (sectional breathing and full yogic breathing, Bhramari, Anulom-vilom, Udgeet); Dhyana. The patient was almost fully compliant. She was regular in taking all the medicines and attended most of the yoga sessions.

Details of the patient's progress and compliance during the course of the treatment are given below in Table 25 .

\begin{tabular}{|c|c|c|c|}
\hline Day & Date & Vital Measures & General Remarks \\
\hline 0 & Oct 24 & $\begin{array}{l}\text { SpO2: } 97 \\
\text { Temp: } 98.5\end{array}$ & $\begin{array}{l}\text { Symptoms at the time of first consultation: } \\
\text { mild cough, body ache, weakness; later she } \\
\text { also reported to be having loose stools and } \\
\text { chest congestion. }\end{array}$ \\
\hline 1 & Oct 25 & $\begin{array}{l}\text { SpO2: } 98 \\
\text { PR: } 122 \\
\text { Temp: } 100.4\end{array}$ & $\begin{array}{l}\text { Experiencing throat irritation since the pre- } \\
\text { vious night - Koflet syrup started; doing } \\
\text { steam inhalation multiple times a day - slight } \\
\text { improvement in congestion; patient devel- } \\
\text { oped fever - took PCM; loose stools and } \\
\text { weakness persist; feeling cold. }\end{array}$ \\
\hline 2 & Oct 26 & $\begin{array}{l}\text { SpO2: } 97-98 \\
\text { PR: } 88-115 \\
\text { Temp: } 97.8-100.1\end{array}$ & $\begin{array}{l}\text { Symptoms persist; experiencing new symp- } \\
\text { toms - irritation/burning sensation in feet, } \\
\text { shivering due to weakness, appetite slightly } \\
\text { reduced. Blood test done on doctor's sug- } \\
\text { gestion - all paramaters found okay. Patient } \\
\text { took PCM due to fever. }\end{array}$ \\
\hline
\end{tabular}




\begin{tabular}{|c|c|c|c|}
\hline 3 & Oct 27 & $\begin{array}{l}\text { SpO2: } 96-99 \\
\text { PR: } 103-116 \\
\text { Temp: } 98.7-99.3\end{array}$ & $\begin{array}{l}\text { Symptoms persist; appetite coming back to } \\
\text { normal; had motion twice. Patient took } \\
\text { PCM due to mild fever. }\end{array}$ \\
\hline 4 & Oct 28 & $\begin{array}{l}\text { SpO2: } 99 \\
\text { PR: } 96-100 \\
\text { Temp: } 98.5-98.6\end{array}$ & $\begin{array}{l}\text { Improvement in chest congestion, stomach } \\
\text { and appetite; no more throat irritation; still } \\
\text { feels slightly cold. }\end{array}$ \\
\hline 5 & Oct 29 & $\begin{array}{l}\text { SpO2: } 99 \quad \text { PR: } 97 \\
\text { Temp: } 98.7\end{array}$ & $\begin{array}{l}\text { Stomach alright; appetite not normal yet; } \\
\text { still feels cold and weak. }\end{array}$ \\
\hline 6 & Oct 30 & $\begin{array}{l}\text { SpO2: } 97-99 \\
\text { PR: } 88-106 \\
\text { Temp: } 98.2-98.6\end{array}$ & $\begin{array}{l}\text { No more congestion; only weakness and cold } \\
\text { feeling persist; patient reported post-nasal } \\
\text { drip. Overall } 75 \% \text { recovery reported. }\end{array}$ \\
\hline 7 & Oct 31 & $\begin{array}{l}\text { SpO2: } 96-98 \\
\text { PR: } 97-98 \\
\text { Temp: } 98.5-98.8\end{array}$ & \\
\hline 8 & Nov 01 & $\begin{array}{l}\text { SpO2: } 95-96 \quad \text { PR: } \\
71-81 \\
\text { Temp: } 98.0-98.5\end{array}$ & Recovery reported; medicines continued. \\
\hline 12 & Nov 05 & $\begin{array}{l}\text { SpO2: } 97-98 \quad \text { PR: } \\
68-73 \\
\text { Temp: } 97.0-97.3\end{array}$ & $\begin{array}{l}\text { Patient found to be in good health during } \\
\text { follow-up. }\end{array}$ \\
\hline
\end{tabular}

Table 25: Progress of patient P23 and evolution of the treatment plan

\section{Detailed treatment plan and progress tracking for P24:}

The patient presented with the following symptoms at the time of the first consultation: Sore throat (mild) with mild pain, weakness, loss of smell, taste.

History of the present illness was taken, which is as follows. The patient developed fever on October 17 (which persisted for about 6 days). Following this, she consulted a doctor on October 19, and was prescribed the following Allopathic medicines: Doxycycline 100 (2 days), Calpol 650 (SOS), Syp. Piritone (2 days); Zincovit, Celin (Vit C) - this was never taken by the patient, Cap. Uprise (D3) (once a week for 2 weeks). She was also advised the following tests: RT-PCR, 
CBC, CRP, NS Agent (dengue). She underwent RTPCR on October 21 and was tested positive. Subsequently she was gven the following Allopathic prescription: Fabiflu 200mg, Calpol, Piritone, Zinconia, Celin - all for 10 days; she was also suggested some breathing exercises. Besides, the patient reported to be doing steam inhalation and gargles 3-4 times a day, and taking home-made kadha prepared with dalchini (cinnamon), laung (clove), ilaichi (cardamom), adrak (ginger), giloy, tulsi (basil), kali mirch (black pepper).

The patient had been taking the following medicines regularly for her comorbidity(ies): Olmizede 40 .

Based on the patient's medical history and the symptoms presented, the following prescription for the Integrative Therapy was given:

Ayurvedic medicines and procedures prescribed: Sanshamani Vati (2 TDS), Laxmivilas Ras (2 BD), Ashwagandha Vati (2 BD), Sarpgandha Vati (1 OD at night after dinner), kadha (to be continued), nasya with Anu teil, usha paan, steam inhalation and gargles - all for 15 days. Some addition was done to the prescription during the course of treatment due to some new symptoms presented by the patient. Also, after recovery from other symptoms, the prescription was appropriately modified to help the patient get over post-recovery weakness. Details of the same have been furnished in Table 26 Yoga Protocol: Sukshma Vyayama (upper and lower body parts); Breathing exercises (4 exercises with 5 iterations in each); asanas: Tadasana, TriyakTadasana, Trikonasana, Ardhachakrasana, Parshva Sukhasana, Utthana Mandukasana, ArdhaUstrasana, Vakrasana, Bhujangasana, Shalabhasana, Ardhahalasana, Pawanmuktasana, Shavasana; Pranayama (sectional breathing and full yogic breathing, Bhramari, Anulom-vilom, Udgeet); Dhyana.

175 The patient was almost fully compliant. She was regular in taking all the medicines and attended most of the yoga sessions.

Details of the patient's progress and compliance during the course of the treatment are given below in Table 26 . 


\begin{tabular}{|c|c|c|c|}
\hline Day & Date & Vital Measures & General Remarks \\
\hline 0 & Oct 24 & $\begin{array}{l}\text { SpO2: } 97-99 \\
\text { Temp: } 97.0-98.7 \\
\text { BP sys: } 120-130 \\
\text { BP dias: } 82-84\end{array}$ & $\begin{array}{l}\text { Symptoms at the time of first consultation: } \\
\text { Sore throat (mild) with mild pain, weakness, } \\
\text { loss of smell, taste. }\end{array}$ \\
\hline 1 & Oct 25 & $\begin{array}{l}\text { SpO2: } 99 \\
\text { PR: } 82-85 \\
\text { Temp: } 98.4 \\
\text { BP sys: } 128-130 \\
\text { BP dias: } 80-82\end{array}$ & $\begin{array}{l}\text { symptoms persist, but overall improvement - } \\
\text { patient reported to be feeling better than the } \\
\text { previous day. }\end{array}$ \\
\hline 2 & Oct 26 & $\begin{array}{l}\text { SpO2: } 99 \\
\text { PR: } 82 \\
\text { Temp: } 98.4 \\
\text { BP sys: } 130 \\
\text { BP dias: } 80-84\end{array}$ & $\begin{array}{l}\text { Patient reported severe body-ache (new } \\
\text { symptom) and weakness, and skipped the } \\
\text { yoga session. }\end{array}$ \\
\hline 3 & Oct 27 & $\begin{array}{l}\text { SpO2: } 99 \\
\text { PR: } 82-84 \\
\text { Temp: } 98.4 \\
\text { BP sys: } 125-130 \\
\text { BP dias: } 82-85\end{array}$ & $\begin{array}{l}\text { Improvement in body-ache; no more sore } \\
\text { throat; weakness persists. }\end{array}$ \\
\hline 4 & Oct 28 & $\begin{array}{l}\text { SpO2: } 99 \\
\text { PR: } 82-84 \\
\text { Temp: } 98.4 \\
\text { BP sys: } 125-130 \\
\text { BP dias:82-84 }\end{array}$ & Symptoms improving; weakness still there. \\
\hline 5 & Oct 29 & $\begin{array}{l}\text { SpO2: } 99 \text { PR: } 82 \\
\text { Temp: } 100.0\end{array}$ & $\begin{array}{l}\text { Reported to be feeling feverish since the pre- } \\
\text { vious night; had fever during the day, but } \\
\text { subsided by late evening - patient took PCM. } \\
\text { She was suggested to take Sudarshan Ghan- } \\
\text { vati ( } 2 \text { BD) in case fever happens again; also } \\
\text { suggested to take } 6-7 \text { glasses of lemon water } \\
\text { per day, do meditation and deep breathing. } \\
\text { The patient reported relief with respect to } \\
\text { loss of smell and taste. }\end{array}$ \\
\hline 6 & Oct 30 & $\begin{array}{l}\text { SpO2: } 99 \quad \mathrm{PR}: 82 \\
\text { Temp: } 97.6\end{array}$ & $\begin{array}{l}\text { Body-ache surfaced again with throat pain } \\
\text { (new symptom); weakness persists. Patient } \\
\text { started taking Sudarshan Ghanvati. }\end{array}$ \\
\hline
\end{tabular}




\begin{tabular}{|c|l|l|l|}
\hline 7 & Oct 31 & & $\begin{array}{l}\text { Body-ache and weakness persist; felt too } \\
\text { drained out during the yoga session and } \\
\text { started sweating - could not complete. Pa- } \\
\text { tient took Sudarshan Ghanvati. }\end{array}$ \\
\hline 8 & Nov 01 & $\begin{array}{l}\text { SpO2:99 PR: } 82 \\
\text { Temp: } 97.6-98.4\end{array}$ & $\begin{array}{l}\text { No symptom except weakness; improvement } \\
\text { in weakness; overall much better. }\end{array}$ \\
\hline 9 & Nov 02 & $\begin{array}{l}\text { SpO2: 99 PR: 82 } \\
\text { Temp: 98.4 }\end{array}$ & $\begin{array}{l}\text { Patient reported to have almost recovered; } \\
\text { medicines continued. }\end{array}$ \\
\hline 12 & Nov 05 & & $\begin{array}{l}\text { Patient found to be doing fine (except mild } \\
\text { weakness) during follow-up. Sanshamani } \\
\text { Vati, Ashwagandha Vati, Sarpagandha Vati } \\
\text { and nasya continued; massage of calf mus- } \\
\text { cles with coconut oil recommended; Sudar- } \\
\text { shan Ghanvati discontinued. }\end{array}$ \\
\hline
\end{tabular}

Table 26: Progress of patient P24 and evolution of the treatment plan

\section{Detailed treatment plan and progress tracking for P25:}

The patient presented with the following symptoms at the time of the first consultation: sore throat, cough, body-ache.

History of the present illness was taken, which is as follows. The patient felt body-ache, throat irritation and feverish around October 18 with temperature fluctuating around $99^{\circ} \mathrm{F}-100^{\circ} \mathrm{F}$. Thereafter, she was prescribed the following Allopathic medicines: Azee 500 (1 OD for 5 days), tab. LCZ HS 1 OD, Dolo 650 SOS.

Based on the symptoms presented, the patient was prescribed following Ayurvedic medicines: Septilin (1 BD), Oritus (1 tsf. TDS with lukewarm water) - for 5 days.

The patient was not fully compliant; she started experiencing loss of taste on October 22 (which lasted 3 days) and remained febrile. Thereafter, she underwent RAT on October 24, and was tested positive. Subsequently, she was given 
also suggested to take Dolo 650 (SOS) - for 15 days.

The patient had been taking the following medicines regularly for her comorbidity: Telmisartan 40mg (1 OD).

The patient consulted again on October 26. Throat irritation and loss of taste had been resolved by then. The following symptoms were observed: fever (fluctuating between $99^{\circ} \mathrm{F}-100^{\circ} \mathrm{F}$ since the onset of symptoms), body-ache, loss of appetite, bad taste/no taste in food, weakness, mild stomach pain with urge to evacuate the bowel 3-4 times in a day (consistency of the stool normal). Based on her medical history and the symptoms presented, the following prescription for the Integrative Therapy was given:

Ayurvedic medicines and procedures prescribed: Sanshamani Vati (1 BD), Laghumalini Vasant (1 BD), Ashwagandha Vati (1 BD), Ayush Kwath (BD), steam inhalation and gargles - all for 15 days.

Yoga Protocol: Breathing exercises (4 exercises with 5 iterations in each); Sukshma Vyayama (upper and lower body parts); asanas: TriyakTadasana, Trikonasana, Ardhachakrasana, Parshva Sukhasana, Sukhasana twist, Utthana Mandukasana (in Sukhasana), Bhujangasana, Shalabhasana, Ardhahalasana, Pawanmuktasana, Shavasana; Pranayama (sectional breathing and full yogic breathing, Bhramari, Anulom-vilom, Udgeet); Dhyana. The patient was almost fully compliant after October 26 (except that she did not take the kwath). She was regular in taking all the medicines and attended most of the yoga sessions.

Details of the patient's progress and compliance during the course of the treatment are given below in Table 27.

\begin{tabular}{|c|c|l|l|}
\hline Day & Date & Vital Measures & General Remarks \\
\hline \hline 0 & Oct 20 & & Symptoms: sore throat, cough, body-ache. \\
\hline 2 & Oct 22 & & Symptoms: loss of taste. \\
\hline 3 & Oct 23 & & $\begin{array}{l}\text { Symptoms: loss of taste. } \\
\text { Symptoms: loss of taste. Underwent RAT - }\end{array}$ \\
\hline 4 & Oct 24 & & $\begin{array}{l}\text { tested positive. } \\
\end{array}$
\end{tabular}




\begin{tabular}{|c|c|c|c|}
\hline 6 & Oct 26 & & $\begin{array}{l}\text { Symptoms: fever, body-ache, weakness, loss } \\
\text { of appetite, mild stomach pain with in- } \\
\text { creased frequency of bowel evacuation and } \\
\text { semi-formed stools. }\end{array}$ \\
\hline 7 & Oct 27 & SpO2: 93 & $\begin{array}{l}\text { All symptoms persist, except that the patient } \\
\text { reported to be constipating - advised to take } \\
\text { trifla or isabgol (that she was habitual of). }\end{array}$ \\
\hline 8 & Oct 28 & $\begin{array}{l}\text { SpO2: } 93-98 \\
\text { PR: } 106 \\
\text { Temp: } 100.0 \\
\text { BP: } 158 / 80\end{array}$ & $\begin{array}{l}\text { Patient reported relief with respect to upset } \\
\text { stomach; very bad taste in mouth, unable } \\
\text { to eat properly; other symptoms (including } \\
\text { fever) persist. }\end{array}$ \\
\hline 9 & Oct 29 & $\begin{array}{l}\text { SpO2: }>95 \\
\text { Temp: } 99.0-99.8\end{array}$ & $\begin{array}{l}\text { All symptoms persist - fever, body-ache, } \\
\text { weakness, bad taste, reduced appetite. }\end{array}$ \\
\hline 10 & Oct 30 & $\begin{array}{l}\text { SpO2: } 96 \\
\text { PR: } 95-100 \\
\text { Temp: } 98.4-99.8 \\
\text { BP sys: } 130-162 \\
\text { BP dias: } 75-85\end{array}$ & $\begin{array}{l}\text { Stomach upset again - semi-formed stools } \\
\text { with increased frequency; trying a eat a little } \\
\text { despite bad taste; extremely troubled by bad } \\
\text { taste and body-ache. Patient reported not to } \\
\text { be taking BP medicine since she got fever - } \\
\text { advised by the doctor to resume. }\end{array}$ \\
\hline 11 & Oct 31 & $\begin{array}{l}\text { SpO2: } 95-98 \\
\text { PR: } 102 \\
\text { Temp: } 97.0-99.4 \\
\text { BP: } 150 / 82\end{array}$ & $\begin{array}{l}\text { Resumed BP medicine. All symptoms per- } \\
\text { sist; patient feeling very weak and tired; took } \\
\text { DOLO for fever in the morning. }\end{array}$ \\
\hline 12 & Nov 01 & $\begin{array}{l}\text { SpO2: } 95 \\
\text { Temp: } 99.0\end{array}$ & $\begin{array}{l}\text { Improvement seen in appetite; did not de- } \\
\text { velop fever during the day despite not tak- } \\
\text { ing any medicine for fever - however had } 99.0 \\
\text { temperature at night. Experiencing extreme } \\
\text { weakness and pain in spine. }\end{array}$ \\
\hline 13 & Nov 02 & $\begin{array}{l}\text { SpO2: } 95 \\
\text { BP: } 144 / 85\end{array}$ & $\begin{array}{l}\text { No fever; improvement with respect to body- } \\
\text { ache and appetite; taste still bad; stomach } \\
\text { still upset; weakness and fatigue persist. }\end{array}$ \\
\hline 14 & Nov 03 & $\begin{array}{l}\text { SpO2: } 95 \\
\text { Temp: } 99.5 \\
\text { BP: } 135 / 80\end{array}$ & Developed fever again. \\
\hline
\end{tabular}




\begin{tabular}{|c|c|c|c|c|}
\hline 15 & Nov 04 & SpO2: 96 & & $\begin{array}{l}\text { No fever, stomach fine; improvement in all } \\
\text { symptoms - body-ache, weakness and taste; } \\
\text { appetite still not normal. Overall } 69 \% \text { recov- } \\
\text { ery reported. }\end{array}$ \\
\hline 16 & Nov 05 & $\begin{array}{l}\text { SpO2: } 96 \\
\text { BP: } 133 / 87\end{array}$ & & No more fever. \\
\hline 17 & Nov 06 & $\begin{array}{l}\text { SpO2: } 98 \\
\text { BP: } 135 / 79\end{array}$ & & $\begin{array}{l}\text { Pain in spine; overall condition same as be- } \\
\text { fore; fatigue (though no fever) after doing } \\
\text { household work - started sweating, feels cold } \\
\text { at times. }\end{array}$ \\
\hline 18 & Nov 07 & & & $\begin{array}{l}\text { Tiredness and weakness still there; taste and } \\
\text { appetite not normal. }\end{array}$ \\
\hline 19 & Nov 08 & $\begin{array}{l}\text { SpO2: } 98 \\
118 / 76\end{array}$ & BP: & $\begin{array}{l}\text { Pain in spine; taste getting better day by } \\
\text { day; weakness persists, appetite not normal; } \\
\text { sweating happens. }\end{array}$ \\
\hline 20 & Nov 09 & $\begin{array}{l}\text { SpO2: } 98 \\
133 / 88\end{array}$ & BP: & \\
\hline 21 & Nov 10 & $\begin{array}{l}\text { SpO2: } 97 \\
138 / 87\end{array}$ & BP: & $\begin{array}{l}\text { Body-ache persists, weakness felt when too } \\
\text { tired after doing household work; taste much } \\
\text { better; appetite normal - felt hungry after } \\
\text { many days. Overall more than } 75 \% \text { recovery } \\
\text { reported. }\end{array}$ \\
\hline 28 & Nov 17 & & & $\begin{array}{l}\text { Status found during follow-up: symptoms no } \\
\text { longer persist; appetite and taste normal, no } \\
\text { more weakness; almost complete recovery re- } \\
\text { ported. }\end{array}$ \\
\hline
\end{tabular}

Table 27: Progress of patient P25 and evolution of the treatment plan

\section{Detailed treatment plan and progress tracking for P26:}

The patient presented with the following symptoms at the time of the first consultation on October 26: Gastric upset, burning sensation in stomach, and was prescribed Amlapittantak Yog, Alsarex, Himcospaz. The next day, on October 
27, he consulted again for cough, when he was prescribed Koflet SF (in addition to the first prescription). The patient underwent RAT and RTPCR on October 28 , both of which turned negative. The patient continued to experience new symptoms, and contacted the doctor again on October 30 for cough and sore throat, when he was prescribed Sitopaladi (1 TDS), Septilin (1 BD), Laxmivilas Ras (1 TDS), Koflet SF (2 tsp TDS with lukewarm water) - all for 5 days; he was also suggested to take PCM (SOS). The doctor also recommended blood test - reports received on Nov. 1 revealed low platelet count. Subsequently, the following medicine was added to his prescription: Orplat (1 BD for 3 days). Meanwhile, other family members of the patient started developing similar symptoms, which led the doctor to suspect COVID. The patient underwent another RTPCR on Nov. 2 at the doctor's suggestion, and was tested positive this time. At this time, the patient presented with the following symptoms: fever, cough with sputum, loose stools. After this, he was prescribed the following Allopathic medicines and supplements: Vitneurin CZS (1 OD), Citravite XT (1 OD) - given on Nov. 3 for 15 days; PCM 650 (1 TDS), Doxycycline 100 (1 OD for 5 days). Besides the patient reported to have been taking Ayush Kwath as a preventive measure.

The patient had been taking the following medicines regularly for his comorbidities: Amloz AT (1 OD), Glucoryl-M1 (1 OD), Thyroxine 50 mcg, Zyloric (1 OD).

Based on the patient's medical history and the symptoms presented, the following prescription for the Integrative Therapy was given.

Ayurvedic medicines and procedures prescribed: Sudarshan Ghanvati (1 TDS), tab. Sitopaladi (1 TDS), syp. Koflet SF (2 tsf TDS with lukewarm water) - for 3 days; Sanshamani Vati (1 BD), Laghumalini Vasant (1 BD), Ashwagandha Vati (1 BD), Chyawanprash (Organic) (1 tsf OD), steam inhalation and gargles - for 15 days; the patient was also advised to continue taking kwath. Some modifications were made to the prescription during the course of treatment based on the condition of the patient.

Yoga Protocol: Sukshma Vyayama (upper and lower body parts); Breathing 
exercises (4 exercises with 5 iterations in each); asanas: Tadasana, TriyakTadasana, Trikonasana, Ardhachakrasana, Parshva Sukhasana, Utthana Mandukasana, ArdhaUstrasana, Vakrasana, Bhujangasana, Shalabhasana, Ardhahalasana, Pawanmuktasana, Shavasana; Pranayama (sectional breathing and full yogic breathing, Bhramari, Anulom-vilom, Udgeet); Dhyana.

The patient was almost fully compliant. He was regular in taking the medicines and attended all the yoga sessions.

Details of the patient's progress and compliance during the course of the treatment are given below in Table 28 .

\begin{tabular}{|c|c|c|c|}
\hline Day & Date & Vital Measures & General Remarks \\
\hline 0 & Oct 26 & & Symptoms: gastric upset. \\
\hline 1 & Oct 27 & & Symptoms: gastric upset, cough. \\
\hline 4 & Oct 30 & & $\begin{array}{l}\text { Symptoms: cough, sore throat. Blood-test } \\
\text { recommended. }\end{array}$ \\
\hline 6 & Nov 01 & & $\begin{array}{l}\text { Symptoms persist; low platelet count ob- } \\
\text { served in blood-test report - Orplat (1 BD) } \\
\text { added to the prescription. }\end{array}$ \\
\hline 7 & Nov 02 & & $\begin{array}{l}\text { Symptoms: fever, cough with sputum, upset } \\
\text { stomach - loose stools. }\end{array}$ \\
\hline 8 & Nov 03 & $\begin{array}{l}\text { SpO2: } 94 \\
\text { PR: } 67 \\
\text { Temp: } 100.0\end{array}$ & $\begin{array}{l}\text { Symptoms: fever, cough with sputum, up- } \\
\text { set stomach - loose stools. SpO2 level be- } \\
\text { low } 95 \text { - Makarasana and Shithilasana rec- } \\
\text { ommended. }\end{array}$ \\
\hline 9 & Nov 04 & $\begin{array}{l}\text { SpO2: } 94-96 \\
\text { PR: } 63-89 \\
\text { Temp: } 98.7-100.0 \\
\text { BP: } 119 / 75\end{array}$ & $\begin{array}{l}\text { Fever, cough with sputum and loose stools } \\
\text { persist. SpO2 still below } 95 \text { - patient again } \\
\text { advised to do Makarasana and Shithilasana }\end{array}$ \\
\hline 10 & Nov 05 & $\begin{array}{l}\text { SpO2: } 95-98 \\
\text { PR: } 64-71 \\
\text { Temp: } 98.6-100.5 \\
\text { BP: } 112 / 79\end{array}$ & $\begin{array}{l}\text { Fever, weakness persist; appetite reduced } \\
\text { (mostly on liquid diet); improvement with re- } \\
\text { spect to cough with sputum; upset stomach - } \\
\text { semi-formed stools with increased frequency. } \\
\text { Reported to have felt drowsiness during the } \\
\text { day, vomited - took allopathic medicines Di- } \\
\text { lgk5e and Pantoprazole gadtro resistance (1 } \\
\text { tab. each). Septilin continued for } 5 \text { days. }\end{array}$ \\
\hline
\end{tabular}




\begin{tabular}{|c|c|c|c|}
\hline 11 & Nov 06 & $\begin{array}{l}\text { SpO2: } 94-97 \\
\text { PR: } 67-74 \\
\text { Temp: } 98.6-100.0\end{array}$ & $\begin{array}{l}\text { Fever persists - develops at night, becomes } \\
\text { normal during the day; fever is accompanied } \\
\text { with weakness; mild dry cough and reduced } \\
\text { appetite persist (mostly on liquid diet); im- } \\
\text { provement with respect to loose stools. }\end{array}$ \\
\hline 12 & Nov 07 & $\begin{array}{l}\text { SpO2: } 98 \\
\text { PR: } 66 \\
\text { Temp: } 98.5 \\
\text { BP: } 118 / 82\end{array}$ & $\begin{array}{l}\text { No more fever; improvement with respect to } \\
\text { weakness. Overall better than the previous } \\
\text { day. }\end{array}$ \\
\hline 13 & Nov 08 & $\begin{array}{l}\text { SpO2: } 98 \\
\text { PR: } 66-96 \\
\text { Temp: } 96.0-97.6 \\
\text { BP sys: } 115-120 \\
\text { BP dias: } 76-80\end{array}$ & $\begin{array}{l}\text { Mild dry cough and slight weakness per- } \\
\text { sist; no more loose stools; appetite normal. } \\
\text { More than } 80 \% \text { recovery reported. Sudar- } \\
\text { shan Ghanvati given again for } 2 \text { days. }\end{array}$ \\
\hline 14 & Nov 09 & $\begin{array}{l}\text { SpO2: } 98-99 \\
\text { PR: } 57-65 \\
\text { Temp: } 97.5 \\
\text { BP sys: } 116-119 \\
\text { BP dias: } 79\end{array}$ & \\
\hline 15 & Nov 10 & $\begin{array}{l}\text { SpO2: } 98 \\
\text { PR: } 65 \\
\text { Temp: } 97.2-97.5 \\
\text { BP sys: } 115-117 \\
\text { BP dias: } 79-81\end{array}$ & \\
\hline 16 & Nov 11 & $\begin{array}{l}\text { SpO2: } 99 \\
\text { PR: } 56-65 \\
\text { Temp: } 97.5 \\
\text { BP sys: } 110-119 \\
\text { BP dias: } 82-86\end{array}$ & \\
\hline 17 & Nov 12 & $\begin{array}{l}\text { SpO2: } 97-99 \\
\text { PR: } 60-62 \\
\text { Temp: } 97.8-98 \\
\text { BP sys: } 101-112 \\
\text { BP dias: } 78-80\end{array}$ & $\begin{array}{l}\text { No more symptoms. Complete recovery re- } \\
\text { ported; occasional mild cough due to pollu- } \\
\text { tion. }\end{array}$ \\
\hline
\end{tabular}


Table 28: Progress of patient P26 and evolution of the treatment plan

\section{Detailed treatment plan and progress tracking for P27:}

The patient presented with the following symptoms at the time of the first con-

ing prescription for the Integrative Therapy was given.

Ayurvedic medicines and procedures prescribed: Sudarshan Ghanvati (1 TDS), Amalpittantak Yog (1 TDS), tab. Unexozim - for 3 days; Sanshamani Vati (1 BD), Laghumalini Vasant (1 BD), Ashwagandha Vati (1 BD), Chyawanprash 1825 (Organic) (1 tsf OD), steam inhalation and gargles - for 15 days; the patient was also advised to continue taking kwath. Some modifications were made to the prescription during the course of treatment based on the condition of the patient.

Yoga Protocol: Sukshma Vyayama (upper and lower body parts); Breathing exercises (4 exercises with 5 iterations in each); asanas: Tadasana, TriyakTadasana, Trikonasana, Ardhachakrasana, Parshva Sukhasana, Utthana Mandukasana, ArdhaUstrasana, Vakrasana, Bhujangasana, Shalabhasana, Ardhahalasana, Pawanmuktasana, Shavasana; Pranayama (sectional breathing and full yogic breathing, Bhramari, Anulom-vilom, Udgeet); Dhyana. 
The patient was almost fully compliant. She was regular in taking the medicines and attended all the yoga sessions.

Details of the patient's progress and compliance during the course of the treatment are given below in Table 29.

\begin{tabular}{|c|c|c|c|}
\hline Day & Date & Vital Measures & General Remarks \\
\hline 0 & Oct 28 & & Symptoms: body-ache. \\
\hline 6 & Nov 03 & $\begin{array}{l}\text { SpO2: } 94 \\
\text { PR: } 97 \\
\text { Temp: } 100.0\end{array}$ & $\begin{array}{l}\text { Symptoms: fever, weakness and loss of ap- } \\
\text { petite. SpO2 level below } 95 \text { - Makarasana } \\
\text { and Shithilasana recommended. }\end{array}$ \\
\hline 7 & Nov 04 & $\begin{array}{l}\text { SpO2: } 98-99 \\
\text { PR: } 87-97 \\
\text { Temp: } 98.7 \\
\text { BP: } 164 / 97\end{array}$ & $\begin{array}{l}\text { Weakness and loss of appetite persist (mostly } \\
\text { on liquid diet); no fever. SpO2 level im- } \\
\text { proved. }\end{array}$ \\
\hline 8 & Nov 05 & $\begin{array}{l}\text { SpO2: } 96-97 \\
\text { PR: } 88-106 \\
\text { Temp: } 98.4-99.0 \\
\text { BP: } 145 / 95\end{array}$ & $\begin{array}{l}\text { Symptoms persist; mild fever observed, } \\
\text { though patient did not feel feverish; largely } \\
\text { on liquid diet. Sudarshan Ghanvati and } \\
\text { Amalpittantak yog continued for } 2 \text { days. }\end{array}$ \\
\hline 9 & Nov 06 & $\begin{array}{l}\text { SpO2: } 97-100 \\
\text { PR: } 80-101 \\
\text { Temp: } 98.6-99.2\end{array}$ & $\begin{array}{l}\text { mild fever relapse; weakness persists; ap- } \\
\text { petite getting normal. }\end{array}$ \\
\hline 10 & Nov 07 & $\begin{array}{l}\text { SpO2: } 98 \\
\text { PR: } 84 \\
\text { Temp: } 98.5 \\
\text { BP: } 148 / 105\end{array}$ & $\begin{array}{l}\text { No more fever; improvement with respect to } \\
\text { weakness. }\end{array}$ \\
\hline 11 & Nov 08 & $\begin{array}{l}\text { SpO2: } 95-97 \\
\text { PR: } 88-90 \\
\text { Temp: } 99.0-99.5 \\
\text { BP sys: } 140-148 \\
\text { BP dias: } 101-105\end{array}$ & $\begin{array}{l}\text { Mild fever, feels weak when in fever; appetite } \\
\text { completely normal. Overall feeling much bet- } \\
\text { ter. Sudarshan Ghanvati continued for } 2 \\
\text { days; Fifatrol (1 BD) given for } 2 \text { days. }\end{array}$ \\
\hline 12 & Nov 09 & $\begin{array}{l}\text { SpO2: } 98-99 \\
\text { PR: } 79-86 \\
\text { Temp: } 98.5-98.6 \\
\text { BP sys: } 130-136 \\
\text { BP dias: } 89-93\end{array}$ & \\
\hline
\end{tabular}




\begin{tabular}{|c|c|c|c|}
\hline 13 & Nov 10 & $\begin{array}{l}\text { SpO2: } 98-99 \\
\text { PR: } 75-83 \\
\text { Temp: } 97.5-98.5 \\
\text { BP sys: } 137-140 \\
\text { BP dias: } 94-95\end{array}$ & \\
\hline 14 & Nov 11 & $\begin{array}{l}\text { SpO2: } 97-98 \\
\text { PR: } 66-82 \\
\text { Temp: } 98.4 \\
\text { BP sys: } 110-134 \\
\text { BP dias: } 82-93\end{array}$ & \\
\hline 15 & Nov 12 & $\begin{array}{l}\text { SpO2: } 98 \\
\text { PR: } 77-83 \\
\text { Temp: } 97.2-98.4 \\
\text { BP sys: } 133-134 \\
\text { BP dias: } 96-101\end{array}$ & Complete recovery reported. \\
\hline
\end{tabular}

Table 29: Progress of patient P27 and evolution of the treatment plan

The patient presented with the following symptoms at the time of the first consultation on November 04: body-ache, mild cough and feverish feeling. He was prescribed the following Ayurvedic medicines: Sudarshan Ghanvati (1 TDS) and Coldab tablets (1 TDS), Septilin (1 BD) - for 2 days. The symptoms persisted and he underwent RAT on November 06, wherein he was tested positive. At this point, the symptoms presented by the patient were: mild cough with sputum, back-ache and loose motions. Subsequently, he was prescribed the following Allopathic supplements: Vitneurin CZS (1 OD), Citravite XT (1 OD) The patient had been taking the following medicines regularly for his comorbidity: Telma 40 (1 OD).

Based on the patient's medical history and the symptoms presented, the following prescription for the Integrative Therapy was given.

1855 Ayurvedic medicines and procedures prescribed: Coldab tablets (1 TDS), Im- 
munocare (1 BD), Septilin (1 BD), syp. Kufrakshak (2 tsf. TDS with lukewarm water) - for 3 days; Sanshamani Vati (1 BD), Laghumalini Vasant (1 BD), Ayush Kwath (BD), steam inhalation and gargles - for 15 days; Ashwagandha Vati (1 BD) was added on November 7. Some modifications were made to the prescription during the course of treatment based on the condition of the patient.

Yoga Protocol: Sukshma Vyayama (upper and lower body parts); Breathing exercises (4 exercises with 5 iterations in each); asanas: Tadasana, TriyakTadasana, Trikonasana, Ardhachakrasana, Parshva Sukhasana, Utthana Mandukasana, ArdhaUstrasana, Vakrasana, Bhujangasana, Shalabhasana, Ardhahalasana, Pawanmuktasana, Shavasana; Pranayama (sectional breathing and full yogic breathing, Bhramari, Anulom-vilom, Udgeet); Dhyana.

The patient was almost fully compliant. He was regular in taking the medicines and attended almost all the yoga sessions.

Details of the patient's progress and compliance during the course of the treatment are given below in Table 30 .

\begin{tabular}{|c|c|c|c|}
\hline Day & Date & Vital Measures & General Remarks \\
\hline 0 & Nov 04 & & $\begin{array}{l}\text { Symptoms: body-ache, mild cough, feverish } \\
\text { feeling. }\end{array}$ \\
\hline 2 & Nov 06 & & $\begin{array}{l}\text { Symptoms: mild cough with sputum, back- } \\
\text { ache, loose stools. }\end{array}$ \\
\hline 3 & Nov 07 & & $\begin{array}{l}\text { Symptoms: cough, loose motions, weakness; } \\
\text { no fever. }\end{array}$ \\
\hline 4 & Nov 08 & $\begin{array}{l}\text { SpO2: } 97-98 \\
\text { PR: } 73-85 \\
\text { Temp: } 97.9 \\
\text { BP: } 145 / 94\end{array}$ & $\begin{array}{l}\text { Cough and weakness persist; no fever; re- } \\
\text { lief from loose motions; reported to be eating } \\
\text { properly and to have slept properly the pre- } \\
\text { vious night after many days. }\end{array}$ \\
\hline 5 & Nov 09 & $\begin{array}{l}\text { SpO2: } 97 \\
\text { PR: } 74 \\
\text { Temp: } 97.4 \\
\text { BP: } 132 / 90\end{array}$ & $\begin{array}{l}\text { Cough and mild weakness persist. The fol- } \\
\text { lowing medicines added for } 2 \text { days: Coldab } \\
\text { tablets, Immunocare and Sudarshan Ghan- } \\
\text { vati. }\end{array}$ \\
\hline 6 & Nov 10 & & Overall improvement reported. \\
\hline
\end{tabular}




\begin{tabular}{|c|c|c|c|}
\hline 8 & Nov 12 & $\begin{array}{l}\text { SpO2: } 98 \\
\text { PR: } 78 \\
\text { BP sys: } 125-149 \\
\text { BP dias: } 87-92\end{array}$ & $\begin{array}{l}\text { The patient reported that } \mathrm{BP} \text { had increased } \\
\text { the previous night, causing headache; still ex- } \\
\text { periencing slight headache and feeling fever- } \\
\text { ish (though temperature is normal); relief } \\
\text { with respect to other symptoms. Yoga Nidra } \\
\text { recommended. }\end{array}$ \\
\hline 9 & Nov 13 & $\begin{array}{l}\text { SpO2: } 97-98 \\
\text { PR: } 75-82 \\
\text { BP sys: } 119-136 \\
\text { BP dias: } 90-93\end{array}$ & $\begin{array}{l}\text { Patient complained of high BP accompanied } \\
\text { with headache - Ayurvedic medicine Cardiol- } \\
\mathrm{H} \text { capsules ( } 1 \mathrm{BD}) \text { added to the prescription } \\
\text { for management of } \mathrm{BP} \text {. }\end{array}$ \\
\hline 10 & Nov 14 & $\begin{array}{l}\text { SpO2: } 98 \text { PR: } 87 \\
\text { BP } 127 / 99\end{array}$ & $\begin{array}{l}\text { Improvement reported in headache; no other } \\
\text { symptoms. }\end{array}$ \\
\hline 13 & Nov 17 & & Complete recovery reported. \\
\hline
\end{tabular}

Table 30: Progress of patient P28 and evolution of the treatment plan

\section{Detailed treatment plan and progress tracking for P29:}

The patient presented with the following symptoms at the time of the first coning prescription for the Integrative Therapy was given. Ayurvedic medicines and procedures prescribed: Sudarshan Ghanvati (1 TDS), 
Laxmivilas Ras (1 TDS), Septilin (1 BD) - 3 days; Sanshamani Vati (1 BD), Laghumalini Vasant (1 BD), Ashwagandha Vati (1 BD), Ayush Kwath (BD), steam inhalation and gargles - for 15 days.

Yoga Protocol: Sukshma Vyayama (upper and lower body parts); Breathing exercises (4 exercises with 5 iterations in each); asanas: Tadasana, TriyakTadasana, Trikonasana, Ardhachakrasana, Parshva Sukhasana, Utthana Mandukasana, ArdhaUstrasana, Vakrasana, Bhujangasana, Shalabhasana, Ardhahalasana, Pawanmuktasana, Shavasana; Pranayama (sectional breathing and full yogic breathing, Bhramari, Anulom-vilom, Udgeet); Dhyana.

The patient was almost fully compliant. She was regular in taking the medicines and attended almost all the yoga sessions. In a follow-up call, she reported to be continuing with yoga practice with the help of the video recording provided to her.

Details of the patient's progress and compliance during the course of the treatment are given below in Table 31 .

\begin{tabular}{|c|c|c|c|}
\hline Day & Date & Vital Measures & General Remarks \\
\hline 0 & Nov 07 & & $\begin{array}{l}\text { Symptoms: body-ache, shivering, sore } \\
\text { throat. }\end{array}$ \\
\hline 1 & Nov 08 & $\begin{array}{l}\text { SpO2: } 97-98 \\
\text { PR: } 75-78 \\
\text { Temp: } 99.7 \\
\text { BP: } 125 / 90\end{array}$ & $\begin{array}{l}\text { Symptoms: Body-ache, weakness, throat ir- } \\
\text { ritation, sneezing; developed fever later dur- } \\
\text { ing the day; eating properly. }\end{array}$ \\
\hline 2 & Nov 09 & $\begin{array}{l}\text { SpO2: } 98 \text { PR: } 90 \\
\text { Temp: } 97.6 \\
\text { BP: } 133 / 97\end{array}$ & $\begin{array}{l}\text { Mild cough developed; weakness and body- } \\
\text { ache persist; nasal/chest blockage - steam in- } \\
\text { halation suggested. }\end{array}$ \\
\hline 3 & Nov 10 & & $\begin{array}{l}\text { Patient started steam inhalation since the } \\
\text { previous day. No more fever; mild cough and } \\
\text { nasal/chest blockage persist; improvement in } \\
\text { weakness and body-ache. }\end{array}$ \\
\hline
\end{tabular}




\begin{tabular}{|c|c|c|c|}
\hline 5 & Nov 12 & $\begin{array}{l}\text { SpO2: } 98-99 \\
\text { PR: } 64-81 \\
\text { BP sys: } 125-131 \\
\text { BP dias: } 93-95\end{array}$ & $\begin{array}{l}\text { Improvement reported in nasal/chest block- } \\
\text { age; relief from weakness and body-ache; } \\
\text { mild cough persists. Overall } 75 \% \text { relief re- } \\
\text { ported. }\end{array}$ \\
\hline 6 & Nov 13 & $\begin{array}{l}\text { SpO2: } 97-98 \\
\text { PR: } 69-71 \\
\text { BP sys: } 133-135 \\
\text { BP dias: } 94-100\end{array}$ & $\begin{array}{l}\text { Much improvement in blockage. No more } \\
\text { symptoms except mild cough. }\end{array}$ \\
\hline 7 & Nov 14 & $\begin{array}{l}\text { SpO2: } 99 \\
\text { PR: } 82 \\
\text { BP: } 124 / 94\end{array}$ & $\begin{array}{l}\text { Patient reported to have almost completely } \\
\text { recovered. No more blockage as evident from } \\
\text { the voice of the patient; mild seasonal cough } \\
\text { remains. }\end{array}$ \\
\hline 10 & Nov 17 & & Complete recovery reported. \\
\hline
\end{tabular}

Table 31: Progress of patient P29 and evolution of the treatment plan

\section{Detailed treatment plan and progress tracking for P30:}

The patient presented with the following symptoms at the time of the first consultation on November 06: body-ache, fever. He reported to have had fever $\left(100^{\circ} \mathrm{F}\right)$ and chills since the previous evening. The following Ayurvedic medicines were prescribed to him: Sudarshan Ghanvati (1 TDS), cap. Immunity (1 TDS) - for 3 days; he was also suggested to take DOLO 650 (SOS). The next day, i.e. on November 07, he underwent RTPCR, and was tested positive. Subsequently, he was prescribed the following allopathic supplements: Vitneurin CZS (1 OD), Citravite XT (1 OD) for 15 days;DOLO 650 (SOS).

The patient had been taking the following medicines regularly for his comorbidity: Sartel H 40 (for past 1 year).

Based on the patient's medical history and the symptoms presented, the following prescription for the Integrative Therapy was given.

Ayurvedic medicines and procedures prescribed: Sudarshan Ghanvati (1 TDS), Immunocare (1 TDS) - 2 days; Sanshamani Vati (1 BD), Laghumalini Vasant (1 BD), Chyawanprash (1 tsf. daily with hot milk) steam inhalation and gargles 
- for 15 days.

Yoga Protocol: Sukshma Vyayama (upper and lower body parts); Breathing exercises (4 exercises with 5 iterations in each); asanas: Tadasana, TriyakTadasana, Trikonasana, Ardhachakrasana, Parshva Sukhasana, Utthana Mandukasana, ArdhaUstrasana, Vakrasana, Bhujangasana, Shalabhasana, Ardhahalasana, Pawanmuktasana, Shavasana; Pranayama (sectional breathing and full yogic breathing, Bhramari, Anulom-vilom, Udgeet); Dhyana.

${ }_{1930}$ The patient was almost fully compliant. He was regular in taking the medicines and attended almost all the yoga sessions. By the end of the treatment, he was strongly convinced about the positive impact yoga could have on his life, and was determined to adopt it in his lifestyle.

Details of the patient's progress and compliance during the course of the treatment are given below in Table 32 .

\begin{tabular}{|c|c|c|c|}
\hline Day & Date & Vital Measures & General Remarks \\
\hline 0 & Nov 06 & & Symptoms: fever, body-ache. \\
\hline 2 & Nov 08 & Temp: 99.3-101.0 & $\begin{array}{l}\text { Symptoms: fever, body-ache, weakness; } \\
\text { SpO2 touching } 95 \text { - Makarasana and Shithi- } \\
\text { lasana suggested. }\end{array}$ \\
\hline 3 & Nov 09 & $\begin{array}{l}\text { SpO2: } 95-97 \\
\text { Temp: } 98.1-99.2\end{array}$ & $\begin{array}{l}\text { Mild fever; mild dry cough and mild weak- } \\
\text { ness; no body-ache; reported to be sleeping } \\
\text { and eating well. }\end{array}$ \\
\hline 4 & Nov 10 & $\begin{array}{l}\text { SpO2: } 96-97 \\
\text { Temp: } 98.3-98.4\end{array}$ & $\begin{array}{l}\text { No more fever; improvement in cough; mild } \\
\text { weakness persists. Overall } 60 \% \text { recovery re- } \\
\text { ported. }\end{array}$ \\
\hline 5 & Nov 11 & $\begin{array}{l}\text { SpO2: } 95-96 \\
\text { Temp: } 98.2-98.4\end{array}$ & \\
\hline 6 & Nov 12 & $\begin{array}{l}\text { SpO2: } 96-97 \\
\text { Temp: } 98.3-98.4\end{array}$ & $\begin{array}{l}\text { Mild cough remains; relief with respect to all } \\
\text { other symptoms. Patient expressed determi- } \\
\text { nation to adopt yoga in his lifestyle. }\end{array}$ \\
\hline 7 & Nov 13 & $\begin{array}{l}\text { SpO2: } 96-97 \\
\text { Temp: } 98.2-98.4\end{array}$ & Condition same as before. \\
\hline
\end{tabular}




\begin{tabular}{|c|c|c|c|}
\hline 8 & Nov 14 & $\begin{array}{l}\text { SpO2: } 96-97 \\
\text { Temp: } 98.3-98.4\end{array}$ & \\
\hline 9 & Nov 15 & $\begin{array}{l}\text { SpO2: } 96-97 \\
\text { Temp: } 98.3-98.4\end{array}$ & Patient reported complete recovery. \\
\hline 10 & Nov 16 & $\begin{array}{l}\text { SpO2: } 96-97 \\
\text { Temp: } 98.2-98.4\end{array}$ & \\
\hline 11 & Nov 17 & $\begin{array}{l}\text { SpO2: } 96-97 \\
\text { Temp: } 98.2-98.4\end{array}$ & \\
\hline 12 & Nov 18 & $\begin{array}{l}\text { SpO2: } 95-96 \\
\text { Temp: } 98.2-98.5\end{array}$ & \\
\hline 13 & Nov 19 & $\begin{array}{l}\text { SpO2: } 96-97 \\
\text { Temp: } 98.3-98.4\end{array}$ & \\
\hline 14 & Nov 20 & $\begin{array}{l}\text { SpO2: } 95-97 \\
\text { Temp: } 98.3-98.4\end{array}$ & Patient continues to be in good health. \\
\hline
\end{tabular}

Table 32: Progress of patient P30 and evolution of the treatment plan

\subsection{Justification of Medicines Used}

Here we provide the ingredients and benefits of the medicines prescribed to the patients.

1940

Giloy is known to have the following properties [66]: Tridosha shamaka (pacifies all the three doshas, i.e. Vata, Pitta, Kapha), Deepan Pachan (Rasa Dhatu, after proper metabolism, converts to the next Dhatu, i.e. Rakta or blood; undigested part of Rasa Dhatu acts as toxin, which is called Ama Dosha; this toxin is responsible for the blockage of micro-channels, i.e. srotas, leading to diseases; the Deepan Pachan property provides for enhancement of digestive fire for digestion of the Ama Dosha, thereby freeing the body of toxins), Jwaraghna (antipyretic), Pramehahara (anti-diabetic), Hrid-daurbalya nashak (useful in the treatment of heart problems), Yakrit vikar nashak (useful in the treatment of liver related 

lems), Raktashodhaka (blood purifier), and Rasayan (rejuvenating and removes weakness).

Ashwagandha has the following properties [66]: Kapha, Vata shamak (pacifies

Pathyadi Kwath 67, 66]: Ingredients: Harad, Baheda, Amla, Neem chhal, Haldi, Giloy. Benefits: Tridosha shamaka (pacifier of all the three doshas, i.e. Vata, Pitta, Kapha; Balya (strength providing); Medhya (nervine tonic); Deepan Pachan creases liver efficiency), Jwaraghna (antipyretic); Mootral (dieuretic, i.e., useful in kidney related problems); Anuloman (carminative); Mridu-rechan (mild purgative); Kasa roga nashak (treats cough related problems); Shwas roga nashak (helps in breathlessness, dyspnea); Swarabheda nashak (helps eliminate hoarseness of voice); Pratishyaya nashak (useful in common cold); Dhatuvardhak (nourishes body tissues, especially Rasa-lymph, plasma, etc., Rakta or blood, Mansa or muscles, Meda or fat and Dhatus); Rochan (removes anorexia, i.e., unwillingness to eat and reduced appetite); Rasayan (removes weakness and is rejuvenating); Pramehaghna (used to treat Madhumeha or diabetes); Yakrit uttejak Rakta shodhak (blood purifier).

Diabecon tablet [68, 69]: Ingredients: Shilajeet, Meshashringi/Gudmara/Madhunashini, Peetashala/Vijaysar/Indian Kino Tree. Benefits: Vata, Pitta, Kapha hara (paci- 
ness); Ama pachak (digests the Ama Dosha, i.e. the toxins in the body); Kapha rog nashak (useful in Kapha related disorders); Mootra roga nashak (useful in urine related problems); useful in Prameha (useful in disorders like diabetes); Kasa roga nashak (used to treat cough related problems); Shwas roga

nashak (useful in breathlessness, dyspnea); especially useful in Madhumeha (diabetes); Madhurya nashak (removes the sweet taste of the tongue); Deepan (appetizer, enhances the digestive fire); Yakrit uttejak (enhances liver efficiency); Pratishyaya nashak (useful in common cold); Kasa roga nashak (used to treat cough related problems); Shwas roga nashak (useful in breathlessness, dyspnea); Rasayan (rejuvenates and removes weakness), Rakta shodhak (blood purifier).

Lavangadi vati [70]: Clove is the main ingredient, which has Kapha dosha balancing, antispasmodic, antiseptic and carminative properties. It is useful in kas roga (cough, sore throat, hoarseness of voice, laryngitis, tracheitis, bronchitis etc.).

Chitrakadi vati [71]: Ingredients - Chitrak Mool Chhal, Pipla Mool, Yavkshar, Sindha Namak (rock salt), Sochar Namak, Kala Namak, Samundra Namak, Sambhar Namak, Sounth, Kali Marich, Pipali Choti, Hing, Ajmod, Chabya, Sajjishar. It is useful in atisar (loss of appetite, indigestion, flatulence).

Arogyavardhini vati [2]: Ingredients - Shudha Mercury, Shudha Gandhak, Louh Bhasma, Abhrak Bhasma, Tamra Bhasma, Shudha Shilajeet, Shudha Guggul, Chitrak Mool, Kutki, Neem Patra Ras, Harad, Baheda, Amla. It is useful in skin and liver disorder, obesity and chronic fever, etc., and gives strength.

Oritus syrup [73]: Oritus Syrup contains Ayurvedic Ingredients (Yashtimadhu $200 \mathrm{mg}$, Vasa $200 \mathrm{mg}$, Tulsi $200 \mathrm{mg}$, Banafsha $200 \mathrm{mg}$, Jufa $100 \mathrm{mg}$, Adrak 100 mg, Bahera $100 \mathrm{mg}$, Kakrasinghi $100 \mathrm{mg}$, Kantakari $50 \mathrm{mg}$, Kulanjan 50 mg, Pippali $50 \mathrm{mg}$, Saunf $6 \mathrm{mg}$, and Pudina Satva $3 \mathrm{mg}$ ). It is useful in the treatment of cough. It open the airways, dissolve mucous and brings about decongestion. 
It also reduces bronchial inflammation and relieves sore throat. Oritus Syrup is used to treat seasonal, allergic and asthmatic cough. It is also useful in treating chronic bronchitis.

Laghumalini Vasant [74]: Dhootapapeshwar Laghumalini Vasant Rasa contains Jasad Bhasma 10 parts, Maricha 5 parts, Navneet 1 part and Bhavana- Nimbu Swarasa as active ingredients. Benefits: potent Vasantakalpa acting on Rasaraktadi Dhatu, Jwaranashak; useful in Rajayakshma, Jeernajwara with its Balya, Rasayan properties.

Sudarshan Ghanvati [75]: Ingredients: Triphala, Haridra, Daruharidra, Kantakari, Brhati, Karcura, Sunthi, Marica, Pippali, Murva, Guduchi, Dhanvayasa, Katuka, Parpata, Musta, Trayamanag, Hrivera, Nimba (Chhal), Puskara, Yasti, ${ }_{2025}$ Kutaja, Yavani, Indrayava, Bharang, Sigru, Saurastri, Vaca, Tvak, Padmaka, Svetacandana, Ativisa, Bala, Salaparn, Prsniparni, Vidanga, Tagara, Citraka, Devadaru, Cavya, Patola, Lavanga, Vamsa, Kamala, Ashwagantha, Tejapatra, Jatiphala, Sthauneya, Vidarikand, Kiratatikta. It is useful in fever. Mahasudarshan Ghanvati [76: Ingredients - Harad (Terminalia chebula), Baheda (Terminalia belerica), Amla (Emblica officinalis), Haldi (Curcuma longa), Daru haldi (Berberi saristata), Kateri Chhoti (Solanum xanthocarpum), Kateri badi (Solanum indicum), Kachoor (Curcuma zedoaria), Sonth (Zingiber officinale), Kali marich (Piper nigrum), Pipal (Piper longum), Piplamool (Piper longum), Murva (Marsdenia tenacissima), Giloy (Tinospora cordifolia), Javasa (Alhagi camelorum), Kutki (Picrorhiza kurroa), Pitpapra (Fumaria indica), Nagarmotha (Cyperus rotundus), Banfsa (Viola odorata), Netrabala (Pavonia odorata), Neem (Azadirachta indica), Pohkar (Inula racemosa), Mulethi (Glycyrrhiza glabra), Kuda (Holarrhena antidysenterica), Ajwain (Trachyspermum ammi), 2040 Indrajav (Holarrhena antidysenterica), Bharangi (Clerodendrum serratum), Sahajan (Moringa pterygosperma), Shudha Phitakari (Alum Pure), Vach (Acorus calamus), Dalchini (Cinnamomum zeylanicum), Padamkhar (Prunus puddum), 
Khas (Vetiveria zizanioides), Chandan shwet (Santalum album), Atis (Aconitum heterophyllum), Khareti (Sida cordifolia), Shalparni (Desmodium gangeticum), Prishinparni (Urari apicta), Viavidang (Embelia ribes), Tagar (Valeriana wallichii), Chitrak (Plumbago zeylanica), Devdaru (Cedrus deodara), Chava (Piper chaba), Patol patra (Trichosanthes dioica), Kalmegha (Andographics paniculata), Karanj (Caesalpinia bonducella), Lavang (Syzygium aromaticum), Vanshlochan (Bambusa - arundinacea), Kamal (Nelumbo nucifera), Kakoli (Lilium polyphyllum), Tejpatra (Cinnamomum tamala), Talis (Abies webbiana), Jivanti (Leptadenia reticulate), and Chirayata (Swertia chirata) as active ingredients. Key benefits/uses of Divya Mahasudarshan Ghanvati are as follows: helps to fight contagious viral infections like swine flu, viral fever, etc.; boosts body's immunity to fight against various infections and diseases; relieves cough and

Kutaj Ghanvati [77: Ingredients - Kutaja bark (Holarrhena antidysenterica), Ativisha (Aconitum heterophyllum). It has potent anti-dysenteric, antidiarrheal, anti-amoebic action and haemostatic properties for treating and curing malabsorption syndrome, intestinal infections and different bleeding disorders. It has Arshoghna (used for treating piles or arsha), Kandughna (used to get relief from itching), and Asthapanopaga (helps in medicated enema) properties. Traditionally, it is also used for curing Atisara (diarrhoea), Jwaratisara (secondary diarrhoea), Asra (blood or blood-related disorders), Pravahika (amebiasis), Trsna (thirst), Kusthahara (skin disorder), Deepana (improves digestion), Vatasruk (treats gout) and Jantujit (relieves from worm infestation).

Laxmivilas ras [78: Ingredients - Sudha Mercury, Sudha Gandhak, Abhrak Bhasma, Kapoor, Javitri, Jaiphal, Vidhara seed, Vidarikand, Satavari, Nagbala, Atibala, Gokhru, Samundra Shosh, Pan Patta Juice. It is useful in cough, cold, asthma attack, rhinitis. 
Basant Kusumakar ras [79]: Ingredients - Swarna Bhasma, Rajat Bhasma,

2095

Septilin tablet 82]: Key Ingredients - Guduchi (Tinospora Cardifolia), Manjistha, Amalaki, Shigru, Yashtimadhu. Key benefits: acts as an immunomodulator, helps in the management of upper and lower respiratory tract infections, provides relief in the case of allergic disorders, helps in sinusitis, helps to im2100

Amynity plus syrup [83]: has 51 herbal extracts and 8 essential oils; strengthens respiratory system, improves digestion and metabolism, powerful anti-oxidant, rejuvenates body tissues. 
Himcocid syrup [84]: Himcocid is an antacid which contains Cowrie Shell Ash and Indian Gooseberry. Cowrie Shell Ash (Varatika) is useful in the treatment of gastritis, hyperacidity, heartburn and duodenitis. Indian Gooseberry (Amalaki) reduces the acid and pepsin content in the stomach. It increases the mucin, cellular mucus and life span of gastric mucosal cells, which helps in healing stomach ulcers. As an antioxidant, Indian Gooseberry protects the gastric mucosa from oxidative damage. Himcocid relieves gastrointestinal discomfort and combats ulcers and gastritis. It neutralizes gastric acid, relieves gaseous distension and bilious and dyspeptic symptoms. It is also effective in: Acid peptic disease (APD), Non-ulcer dyspepsia, Gastroesophageal reflux disease (GERD), Heartburn.

Diarex syrup [85: Ingredients - Coneru and Bael. Coneru (Kutaja) is a potent astringent that is helpful in the treatment of amoebic dysentery and diarrhoea. It inhibits the growth of salmonella (the genus that causes salmonellosis) and shigella (the genus that causes dysentery). Bael (Bilva) fruit has high tannin content, which makes it an effective treatment for dysentery and diarrhea. The fruit exhibits antibacterial and antiviral activity against pathogens, which cause intestinal infections such as infectious diarrhea. Diarex syrup is an antimicrobial and antidiarrheal. Diarex works in acute and chronic diarrhea, acute and chronic amoebiasis and as an adjuvant to the treatment of irritable bowel syndrome (IBS). It works as an antioxidant, has anti-inflammatory and demulcent properties, and antispasmodic action.

Koflet-SF syrup [86]. Key ingredients: Tulsi, Yashtimadhu. Benefits: works as antitussive, antiseptic, anti inflammatory and cough suppressant; reduces the viscosity of bronchial secretions and facilitates expectoration; demulcent action, soothes respiratory passages; Basil possesses potent anti-histaminic properties, so it protects against pollen-induced brochospasms, catarrh and bronchitis due to its varied pharmacological properties; Licorice has antitussive, expectorant 
and immune-enhancing properties that are helpful for relieving cough.

Kutajarishta 87]: Ingredients - Kutaj (Holarrhena antidysenterica),Munnaka (Vitis vinifera), Mahua (Madhucaindica), Ghambhari (Gmelinaarborea), Dhaiful (Woodfordiafruticosa), Gudd (Jaggery). Benefits - Cures diarrhoea, dysentery, malabsorption and fever.

Jirakadyarishta 88]: Ingredients - Cuminum cyminum, Woodfordia fruticosa, Zingiber officinale, Myristica fragrans, Cyperus rotundus, Elettaria cardamomum, Cinnamomum tamala, Mesua ferrea, Piper cubeba, Eugenia caryophyllus. Benefits - Treats gastro-intestinal discomfort caused by parasites and indigestion, and low pitta conditions related to poor circulation and blood toxicity. The principal ingredients are soothing to the stomach, known to reduce flatulence, and to have anti-bacterial, anti-fungal, and anti-viral properties. Cumin and ginger help with the mitigation and release of excess gas in the colon and intestines. Dhawa is a strong cleansing agent both of the blood and G-I tract. Cardamon, Cloves (Eugenia Ca.), and Cinnamon are blood cleansing and soothing for digestion, while Ironwood and Piper Cubeba are effective against dysentery and haemorrhoidal conditions.

2155

Vasavaleh [89]: Ingredients - Vasa, Adusa, Adhatoda, Vasica, Sugar, Long Pepper Powder, Honey. Uses - treatment of cough, asthma, bronchitis, pain in abdomen, bleeding disorders and fever. Traditional Indications: rajayakshmachronic bronchitis, tuberculosis, kasa - cold, cough, shwasa - asthma, respiratory disorders involving difficulty in breathing.

Jufex Forte [90: Ingredients - Amla (Phyllanthus emblica L.), Draksh (Vitis vinifera L.), Vasa (Adhatoda vasica), Tulsi (Ocimum sanctum), Apamarga (Achyranthes aspera L.), Haritaki (Terminalia chebula Retz), Baheda (Terminalia bellirica (Gaertn.) Roxb.), Sheetal Chini (Piper cubeba), Pippali (Piper longum), Sunthi (Zingiber officinale), Alsi (Linum usitatissimum Linn.), Dal- 
chini (Cinnamomum zeylanicum), Tejpatra (Cinnamomum macrocarpum), Guduchi (Tinospora cardifolia), Kalimirch (Piper nigrum L.), Kalaunji (Nigella sativa L. ), Kaddi patta (Murraya koenigii (L.) Spreng), Saunf (Foeniculum vulgare), Amaltas (Cassia fistula L.), Naushadar (Ammonium chloridis ), Gojiha (Onosma bracteatum Wall.), Jufa (Hyssopus officinalis), Kakrasingi (Pistacia integerrima), Gul Banafsha (Viola odorata), Kulanjan (Alpinia galanga (L.) WILLD.), Anjeer (Ficus carica L.), Khubbaji (Malva sylvestris), Talish Patr (Abies bebiana), Khatmi (Althaea officinalis L.), Kaiphal (Myrica esculenta Buch.), Sudh Suhaga 2175 (Purified Borax), Lasora (Cordia dichotama), Pushkarmool (Inula racemosa). Benefits - respiratory tract infections of viral origin, recurrent sore throat, allergic cough, cough associated with chronic bronchitis, pharyngitis, laryngitis.

Ayush Kwath [91]: Ingredients - Tulsi (basil), Saunth (dried ginger), Krishna Marich (black pepper), Dalchini (cinnamon). Benefits - Increases natural immunity, improves respiratory health, boosts digestion.

Nagaradi Kwath [92]: Ingredients - Ginger, Giloy, Haritaki. Benefits - Used in all types of fever.

2185

Kantakari Avaleha 93]: Ingredients - Kantakari, Guduchi, Chavya, Chitraka, em Musta, Karkatashrungi, Pepper, Long pepper, Ginger, Dhanvayasa, Bharangi, Rasna, Shati, Sugar, Sesame oil, Vanshalochana. Uses - useful in respiratory ailments, acts as natural bronchodilator and mucolytic; used in the treatment of bronchitis, COPD, cough, hiccups and cold.

Talishadi churna 94]: Ingredients - Abies Webbiana (Talispatra or Indian Silver Fir), Black Pepper (Piper Nigrum), Sonth - Zingiber Officinale, Pippali (Long Pepper) - Piper Longum, Vanshlochan (Tabashir) - Bamboo Manna, Green ${ }_{2195}$ Cardamom (Elaichi), Dalchini (cinnamon), Misri (crystallized sugar lumps). Benefits - has antitussive, bronchodilator, expectorant (mild), mucolytic, antiinflammatory (effect appear on respiratory tract), antiviral, antibacterial, an- 
timicrobial, carminative properties; is useful in asthma, cough, common cold, chronic bronchitis, whooping cough (pertussis), upper respiratory tract infections, gas or flatulence, bloating, abdominal distension, irritable bowel syndrome, loss of appetite.

Amalaki churna [95]: Ingredients - Indian gooseberry fruit, Indian gooseberry juice, Honey, Sugar, Clarified butter. Uses - useful in constipation fever, cardiac disorders, eye disorders, bleeding ailments, throat diseases, diabetes, urinary tract disorders, digestive disorders; it rejuvenates.

Aloe-vera + Amla juice [96: Ingredients - Aloe-vera, Amla. Benefits - Amla is a natural source of Vitamin $\mathrm{C}$, which is crucial for immunity, healthy hair and eyesight. Amla juice is also an effective heart and liver tonic that helps detoxify and purify the body. A natural anti-oxidant and blood purifier, Aloe Vera strengthens the immune system and improves digestion. It is a good skin tonic and anti-aging agent.

Swarna vasant malti ras [97: Ingredients - Swarn Bhasma, Moti Bhasma, Yasad Bhasma, Sudh Hing (Ferula asafetida), Kali Mirch (Piper nigrum), Nimbu Ras (Citrus medica), Butter. Benefits - useful in curing fever (chronic), lungs related problems, digestion, cough and cold, improves the immune system.

2220 Sitopaladi Tablets [98: Ingredients - Misri (crystallized sugar lumps), Vanshlochan, Pippali (Piper Longum; long pepper), Elaichi (Elettaria Cardamomum; Green Cardamom), Dalchini (Cinnamomum Zeylanicum; Cinnamon). Medicinal Properties: antitussive, immunomodulatory, anti-inflammatory, antimicrobial - antibacterial, adaptogenic, antacid, antiulcerogenic, appetizer and 2225 digestive stimulant, antioxidant, mild antiviral, bronchodilator, carminative, demulcent, detoxifier, febrifuge, tonic. Benefits - useful in diseases related to respiration, digestion and immunity; primarily, restores the natural functions of respiratory, digestive, immune and many other systems of the body. 
Astha 15 syrup [101, 102]: Ingredients - Dhawai (Woodfordia fruticose; fire flame bush or red bell bush), Kantakari (Solanum xanthocarpum; yellow berried nightshade), Vasaka (Adhatoda vasica; malabar nut), Babul/Kikar (Acacia arabica; Mimosaceae), Elaichi (Elettaria cardamomum; Cardamom), Kali mirch (Piper 2250 (Zingiber officinale; Ginger), Kutaj (Holarrhena antidysenterica; Kurchi), Haldi (Curcuma longa; Turmeric), Lavang (Syzygium aromaticum; Clove), Safed Aak (Calotrophis gigantea; Crown Flower), Chota chirayata (Enicostema littorale; Indian Whitehead), Pipli (Piper longum; Long pepper). Benefits - controls com2255 mon cold, coryza, sneezing, nasal obstruction; reduces incessant tickling in the throat pit with dry teasing cough, dyspnoea, aphonia, and sore throat; facilitates expectoration.

Astha 15 capsules 103]: Ingredients - Dhauta (Woodfordia fruticosa); Katai 
2285

Coldab tablets [107]: Ingredients: Sudarshan Churna Ext., Tribhuvankirti Ras, em Laxmivilas Ras, Godanti Bhasma. Benefits: useful in upper throat infection and allergic condition; works as an antibiotic; protects lungs and bronchi from viral infection. (Achyranthus aspera); Adrak (Zingiber officinalis); Kura (Holarrhena antidysentarica); Haldi (Curcuma longa); Laung (Syzygium aromaticum); Laung Oil (Syzygium aromaticum Oil); Safad-AK (Calotropis procera); Chhota Kirayata (Enicostemma littorale); Pippli (Piper longum). Benefits - exhibits unique bronchodilatory and anti-inflammatory effect; dyspnoea, wheezing, cough, expectoration and sleep disturbances are significantly reduced.

Amlapittantak yog [104]: Ingredients: Abhrak bhasma (Mica Calyx), Tamra bhasma (Calcines copper), Mandoor bhasma (Iron slag), Shankh bhasma (Conch shell), Amlaki (Indian gooseberry), Bach and Garlic. Benefits - used in the treatment of gastritis and dyspepsia etc.; acts as an antacid, carminative and appetiser; gives relief from acidity.

Bresol tablets [105]: Ingredients: Tulsi (Basil), Vasaka (Malabar nut), Haldi (Turmeric). Benefits: manages symptoms associated with respiratory disorders; has mucolytic (reducing the viscosity of mucus) and bronchodilatory (decreasing resistance in the breathing airways) properties which help in liquefying and relieving nasal and bronchial congestion; its antimicrobial action combats infections caused by gram-positive and gram-negative bacteria.

Sarpagandha vati [106]: Ingredients - Sarpagandha (Rauwolfia serpentina). Benefits: useful in hypertension, heart diseases, insomnia, epilepsy, psychotic disorders such as delusions, hallucinations, schizophrenia etc. 
Kufrakshak syrup [108]: Ingredients: Kantakari, Vasa, Mulethi, Tulsi, Sonth, Giloy, Gul Banafsha, Funaab, Jufa, Pippali, Bahera, Saunf, Kakarashingi, Dalchini, Lavanga, Pudina Satva and Shuddh Madhu. Benefits: useful in the treatment of seasonal, allergic and asthmatic cough; opens the airways, dissolves mucous and brings about decongestion. It also reduces bronchial inflammation and relieves sore throat.

Immunocare tablets [109]: Ingredients: Bala (Sida cordifolia), Giloya (Tinospora cordifolia), Ashwagandha (Withania somnifera), Tulsi (Ocimum sanctum), Prawal pishti (Micronised coral). Benefits: has anti-oxidant properties that reduces inflammation and slows skin aging; helps to boost immunity and stamina; promotes detoxification; ensures healthy aging, removes blemishes and pigmentation for a smooth, radiant skin.

Cardiol- $H$ tablets [110]: Ingredients: Sarpagandha (Rauwolfia serpentina), Jatamamsi (Nardostachys jatamansi), Gokshuradi guggulu. Benefits: controls high blood pressure and prevents associated symptoms such as headache, anxiety, sleeplessness, stress, stroke and depression; improves coronary circulation and tones up the heart by providing natural cardiac glucosides; prevents constriction and hardening of the blood vessels; .

Unexozim Forte tablets [111]: Ingredients: Saunf, Zeera, Kali Mirch, Sonth, Pipli, Ajwain, Pudina, Kalaunji, Hing, Swarjika Kashar, Nishoth, Kodi Bhasam, Shankh Bhasam. Benefits: treats flatulence, dyspepsia, hyper-acidity, gastrointestinal spasms, constipation.

Immunity capsules [12]: Ingredients: Katuki (Picrorhiza Kurroa), Krishna Tulsi (Ocimum sanctum), Vana Tulsi (Ocimum gratissimum). Benefits: helps to strengthen the immune response to illnesses and infection; offers relief from 2320 cold, flu and other respiratory tract infections. 
Orplat tablets [113]: Ingredients: Carica Papaya Extract and Giloy (Tinospora Cordifolia). Benefits: used in the treatment of dengue fever, dengue shock syndrome and dengue haemorrhagic fever.

2325

Sanshamani vati [114]: Ingredients: Giloy Ghan, Loha Bhasma, Abhrak Bhasma and Swarna Makshik Bhasma. Benefits: highly effective in the treatment of chronic fevers; contains mild antipyretic and anti-inflammatory properties which make it useful for reducing swelling and relieving rheumatism and arthritisrelated pain; improves digestion and removes the disease-causing toxins.

Bacnil capsules [115]: Ingredients: amla, bibhitak, daruharidra,amrita, kirattikta, vidang, haldi, Pathyadi kwath ghan, parpatak and kuberax. Benefits: offers protection against various gastro-intestinal infections; prescribed for diarrhea, bacterial infections caused by gram negative or gram positive bacteria; eradicates pathogenic bacteria; offers anthelmintic and anti-salmonella activity; potentiates the non-specific immune response of the body; offers antipyretic, anti-inflammatory properties.

Softovac 116]: Ingredients: Isabgol, Sonamukhi, Harad, Amaltas, Mulethi, Gulab Jal, Saunf, Saunf Taila, Sharkara, Sodium Benzoate. Benefits: works as a natural remedy for digestive problems; softens the stools and relieves constipation; helps in keeping the digestive system healthy; treats indigestion and removes symptoms such as stomach discomfort or abdominal pain; regulates bowel movement and keeps and activates the secretion of digestive juices thereby improving digestion.

Chyawanprash 117]: Ingredients: Amalaki (Emblica officinalis), Draksa (Vitis vinifera), Ela (Elettaria cardamomum), Pippali (Piper longum), Patra (Cinnamomum tamala), Tvak (Cinnamomum zeylanicum), Abhaya (Terminalia chebula), Amrita (Tinospora cordifolia), Bilva (Aegle marmelos), Agimantha (Clerodendrum phlomidis), Syonaka (Oroxylum indicum). Benefits: a good source of 
calcium; strengthens the immune system; good for the cardiovascular system; purifies blood and removes toxins; improves skin complexion; aids in digestion and maintains balance of stomach acids; relieves constipation and maintains a healthy urinary system; enhances memory; promotes overall health of the body.

Alsarex [118: Ingredients: Amalaki (Emblica officinalis), Shatavari (Asparagus racemosus), Yastimadhu (Glycyrrhiza glabra), Usheer, Udumbar. Benefits: natural antacid - helps in hyperacidity and indigestion; relieves nausea, vomiting and acid regurgitation; accelerates ulcer healing process .

Himcospaz capsules [119]: Ingredients: Ajamoda (Celery), Sunthi (Ginger). Benefits: relieves gastrointestinal tract spasms; acts as an anti-inflammatory; mild analgesic and antispasmodic; recommended for improving digestion, controlling diarrhea and treating pain associated with menstrual cramps.

Oro-T 120]: Ingredients: Haridra (Turmeric), Madhu (Honey), Triphala consisting of three fruits, viz. Amalaki (Phyllanthus emblica), Bibhitaki (Terminalia belleirica) and Haritaki (Terminalia chebula). Benefits: owing to its constituents Oro-T prevents secondary infections, works against inflammation; exerts mucoprotective, anti-inflammatory, antibacterial, wound-healing, antioxidant, radioprotective actions. 